Florida International University FIU Digital Commons

11-13-2009

\title{
A Thermally Wavelength-tunable Photonic Switch Based on Silicon Microring Resonator
}

Xuan Wang

Florida International University, xwang001@fiu.edu

DOI: $10.25148 /$ etd.FI09121602

Follow this and additional works at: https:// digitalcommons.fiu.edu/etd

Part of the Electromagnetics and Photonics Commons

\section{Recommended Citation}

Wang, Xuan, "A Thermally Wavelength-tunable Photonic Switch Based on Silicon Microring Resonator" (2009). FIU Electronic Theses and Dissertations. 137.

https://digitalcommons.fiu.edu/etd/137

This work is brought to you for free and open access by the University Graduate School at FIU Digital Commons. It has been accepted for inclusion in FIU Electronic Theses and Dissertations by an authorized administrator of FIU Digital Commons. For more information, please contact dcc@fiu.edu. 


\title{
FLORIDA INTERNATIONAL UNIVERSITY
}

Miami, Florida

\section{A THERMALLY WAVELENGTH-TUNABLE PHOTONIC SWITCH BASED ON SILICON MICRORING RESONATOR}

\author{
A dissertation submitted in partial fulfillment of the \\ requirements for the degree of \\ DOCTOR OF PHILOSOPHY \\ in \\ ELECTRICAL ENGINEERING \\ by
}

Xuan Wang

2009 
To: Dean Amir Mirmiran

College of Engineering and Computing

This dissertation, written by Xuan Wang, and entitled A Thermally Wavelength-tunable Photonic Switch Based on Silicon Microring Resonator, having been approved in respect to style and intellectual content, is referred to you for judgment.

We have read this dissertation and recommend that it be approved.

\begin{tabular}{rr}
\hline Wonbong Choi \\
\hline Chen Liu \\
\hline Nezih Pala \\
\hline Yuriy A.Vlasov \\
\hline Chunlei Wang \\
\hline Roberto R. Panepucci, Major Professor
\end{tabular}

Date of Defense: November 13, 2009

The dissertation of Xuan Wang is approved.

Dean Amir Mirmiran

College of Engineering and Computing

Dean George Walker University Graduate School

Florida International University, 2009 
(C) Copyright 2009 by Xuan Wang

All rights reserved. 


\section{DEDICATION}

I dedicate this dissertation to my family, especially to my beloved wife, Lu, for making this work meaningful. I also dedicate this work to my little baby, Mario, for bringing this work new meaning; and to my parents for their unfailing love, support, and encouragement. 


\section{ACKNOWLEDGMENTS}

I would like to thank my advisor, Dr. Roberto R. Panepucci, for his great mentoring and guidance throughout the whole period of my graduate study. His passion on research has inspired me a lot. I would also like to show my gratitude to other committee members, Dr. Wonbong Choi, Dr. Chen Liu, Dr. Nezih Pala, Dr. Yuriy A. Vlasov and Dr. Chunlei (Peggy) Wang for evaluating this dissertation.

Regarding the work presented in this dissertation, I would like to acknowledge the other members of the Nanophotonics Research Group for their valuable collaboration and input. Specifically, I would like to thank Dr. Jose A. Martinez for the initial work in Comsol thermal simulation and heater design, Dr. Tao Liu for discussion on optical simulation and Dr. Magdalena Nawrocka for her collaboration and sharing her photonic expertise.

The devices in this dissertation were fabricated by the author in the Cornell Nanofabrication Facility (CNF) and at FIU's Advanced Materials Engineering Research Institute (AMERI). I would like to thank many of the staff members in these two facilities. Especially, I would like to thank Alan R. Bleier and John Treichler at CNF for training and support on Ebeam lithography and Neal Ricks for training on the tools used in AMERI.

This work was supported in part by the AFOSR under FA9550-05-1-0232, New Span Optoelectronics Inc. and National Science Foundation Award No. 0446571. The author was supported by FIU's Dissertation Year Fellowship Award from September 2008 to August 2009. 
ABSTRACT OF THE DISSERTATION

A THERMALLY WAVELENGTH-TUNABLE PHOTONIC SWITCH BASED ON SILICON MICRORING RESONATOR

by

Xuan Wang

Florida International University, 2009

Miami, Florida

Professor Roberto R. Panepucci, Major Professor

Silicon photonics is a very promising technology for future low-cost highbandwidth optical telecommunication applications down to the chip level. This is due to the high degree of integration, high optical bandwidth and large speed coupled with the development of a wide range of integrated optical functions. Silicon-based microring resonators are a key building block that can be used to realize many optical functions such as switching, multiplexing, demultiplaxing and detection of optical wave. The ability to tune the resonances of the microring resonators is highly desirable in many of their applications.

In this work, the study and application of a thermally wavelength-tunable photonic switch based on silicon microring resonator is presented. Devices with $10 \mu \mathrm{m}$ diameter were systematically studied and used in the design. Its resonance wavelength was tuned by thermally induced refractive index change using a designed local microheater. While thermo-optic tuning has moderate speed compared with electro-optic and all-optic tuning, with silicon's high thermo-optic coefficient, a much wider wavelength tunable range can be realized. The device design was verified and optimized by optical 
and thermal simulations. The fabrication and characterization of the device was also implemented. The microring resonator has a measured FSR of $\sim 18 \mathrm{~nm}$, FWHM in the range $0.1-0.2 \mathrm{~nm}$ and $\mathrm{Q}$ around 10,000. A wide tunable range $(>6.4 \mathrm{~nm})$ was achieved with the switch, which enables dense wavelength division multiplexing (DWDM) with a channel space of $0.2 \mathrm{~nm}$. The time response of the switch was tested on the order of $10 \mu \mathrm{s}$ with a low power consumption of $\sim 11.9 \mathrm{~mW} / \mathrm{nm}$. The measured results are in agreement with the simulations.

Important applications using the tunable photonic switch were demonstrated in this work. $1 \times 4$ and $4 \times 4$ reconfigurable photonic switch were implemented by using multiple switches with a common bus waveguide. The results suggest the feasibility of on-chip DWDM for the development of large-scale integrated photonics. Using the tunable switch for output wavelength control, a fiber laser was demonstrated with Erbium-doped fiber amplifier as the gain media. For the first time, this approach integrated on-chip silicon photonic wavelength control. 


\section{TABLE OF CONTENTS}

CHAPTER

PAGE

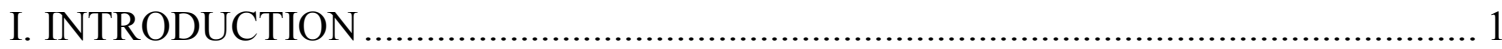

1.1 Transition from Electronics to Photonics in High-speed Communications............... 1

1.2 Silicon-based Integrated Photonics..................................................................... 3

1.3 Integrated Photonics towards DWDM Applications ............................................. 4

1.4 Choosing Silicon for Wavelength Tuning .............................................................. 6

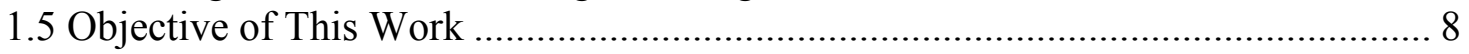

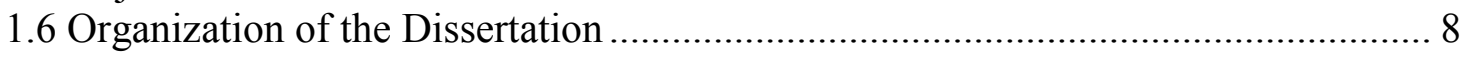

II. THEORY OF MICRORING RESONATOR_...................................................... 10

2.1 Introduction of Microring Resonator .............................................................. 10

2.2 Model of Microring Resonator with Standard Configuration.................................... 11

2.3 Parameters of Microring Resonator .............................................................. 15

2.3.1 Free spectral range (FSR) ………....................................................... 16

2.3.2 Full width at half maximum (FWHM) ....................................................... 16

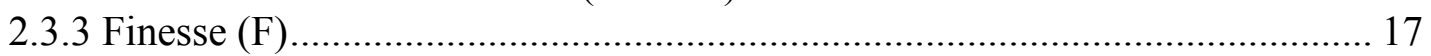

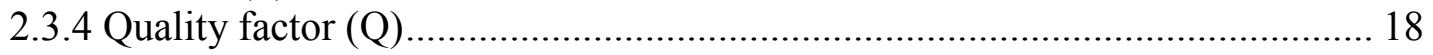

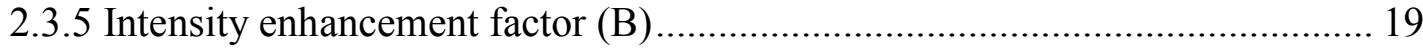

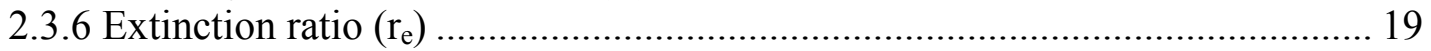

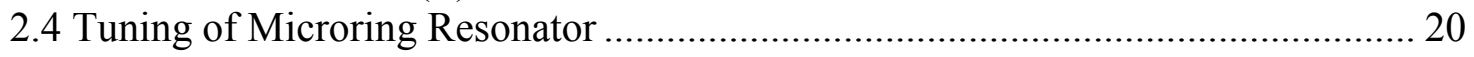

2.5 Thermooptic Wavelength Tuning of Microring Resonator .................................... 23

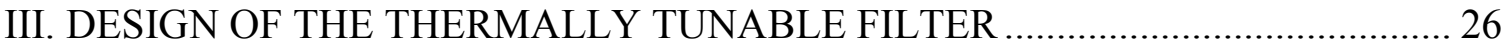

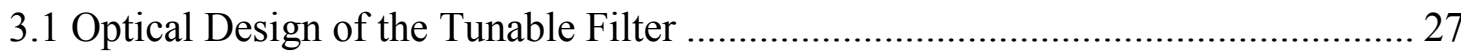

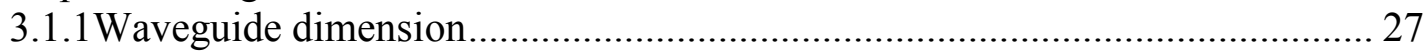

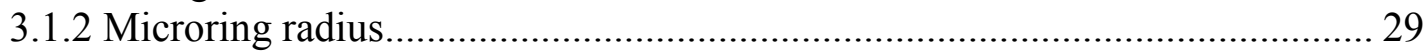

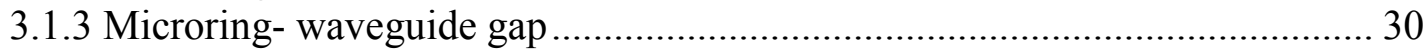

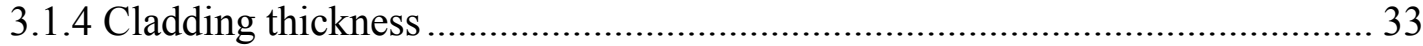

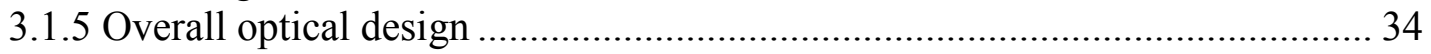

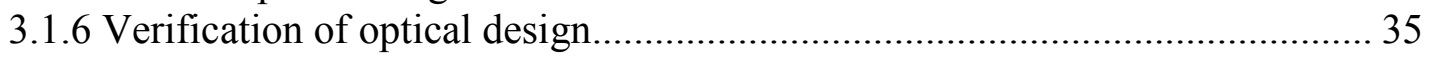

3.2 Thermal Design of the Wavelength-tunable Filter ................................................. 39

3.2.1 S-shape micro-heater design and verification.................................................... 39

3.2.2 Ring-shape micro-heater design and verification ........................................... 48

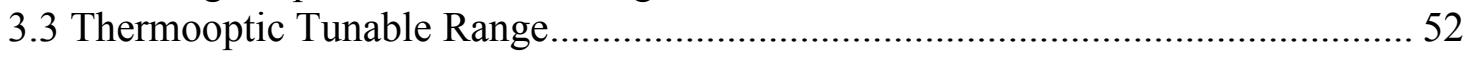

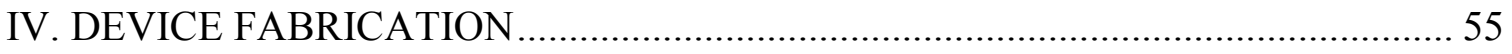

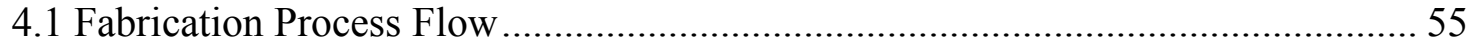

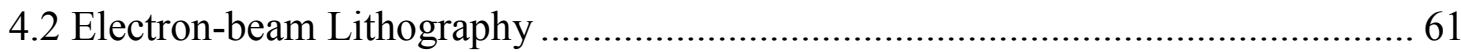

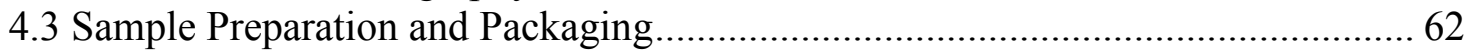

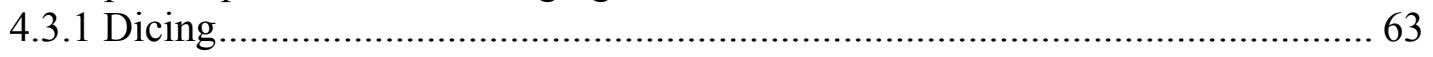

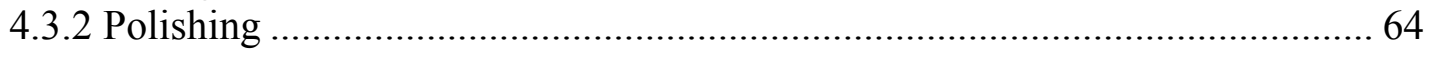

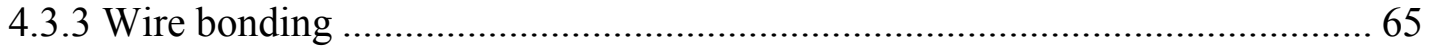




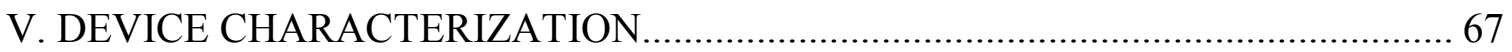

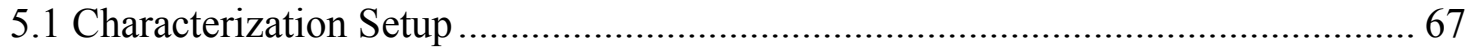

5.2 Optical Spectral Response Measurements and Analysis ..................................... 71

5.3 Wavelength Tuning by Controlling Substrate Temperature ................................ 76

5.4 Wavelength Tuning by Micro-heater......................................................... 78

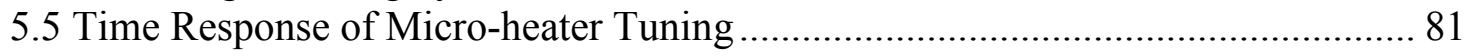

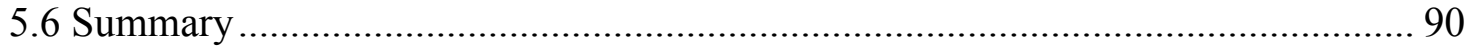

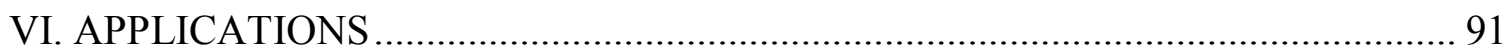

$6.11 \times 4$ and 4x4 Reconfigurable Photonic Switch................................................. 91

6.2 On-chip Wavelength Control of Optical Fiber Laser ......................................... 99

VII. CONCLUSION AND FUTURE WORK .......................................................... 107

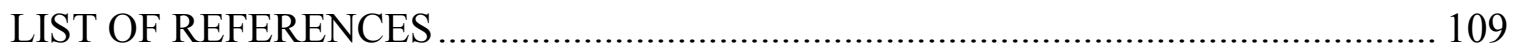

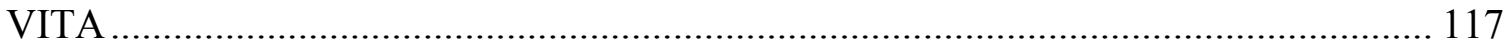




\section{LIST OF TABLES}

TABLE

PAGE

Table 5.1 Summary of TE mode parameters …................................................... 71

Table 5.2 TM Summary of TM mode parameters ...................................................... 71

Table 5.3 Comparison of optical properties measured in this work and in other

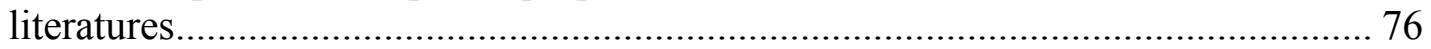

Table 5.4 Comparison of performances of silicon-based thermooptic switch................. 90

Table 6.1 Resonant wavelength peaks of the microring resonators and the resistances of their corresponding micro-heaters ......................................................... 96

Table 6.2 Heating currents determined to tune the microring resonators to the corresponding wavelength channels with the current set marked in bold for the switching configuration shown in Figure 6.4 
Figure 2.1 Model of a standard add/drop type microring resonator

Figure 2.2 Calculated drop port transmission spectra of a microring resonator $(\alpha=$ $0.999, \kappa^{2}=0.02, \mathrm{r}=5 \mu \mathrm{m}$ and $n_{g}=4.21$ ) with (a) tunable resonator loss, (b) tunable resonant wavelength, and (c) tunable coupling ratio.

Figure 3.1 Simulated field distributions for fundamental (a) TE and (b) TM modes at $1550 \mathrm{~nm}$ wavelength. Dark lines shows the boundary of the silicon core.

Figure 3.2 (a) Schematic of the simulated structure with a straight waveguide coupled with a $5 \mu \mathrm{m}$-radius microring. The green boxes are the power monitors. (b) Simulated electrical field distribution on cross-section of the device demonstrating the coupling between straight waveguide and microring. Color indicates the amplitude of electrical field $\mathrm{E}_{\mathrm{x}}$

Figure 3.3 Power coupling coefficient and corresponding Q for different gap width...... 32

Figure3.4 Power coupling coefficient of (a)TE mode and (b) TM mode between straight waveguide and a $5 \mu \mathrm{m}$-radius microring with gap of $200 \mathrm{~nm}$....................... 33

Figure 3.5 Optical design of the tunable filter chip. Inset: magnified picture of the design of the microring resonator

Figure 3.6 Schematic of simulated structure in FDTD simulation of the microring with input and output waveguides in perpendicular.....

Figure 3.7 Simulated through port and drop port spectra of (a) TE mode (b) TM mode.

Figure 3.8 (a) Simulated electrical field $E_{\mathrm{x}}$ distribution of the device at the center plane of the waveguide and the ring (b) Simulated time response of the through port power and the drop port power. The time scale shows the free space propagation length in $\mu \mathrm{m}$. The simulation was done with continuous wave input at a resonance wavelength of $1.5584 \mu \mathrm{m}$

Figure 3.9 Design of micro-heater, feedlines and electrode pads. Inset: magnified picture of the designed micro-heater aligned with the microring .............................. 40

Figure 3.10 Design for the thermal simulation using Comsol Multiphysics

Figure 3.11 Simulated temperature distributions of (a) the whole device (b) $x-z$ plane cross-section at the geometry center of the microring (c) along the microring. All temperatures in Kelvin. The coordinate is shown in (a). 
Figure 3.12 (a) Simulated time response of the temperature change of the microring; (b) Normalized simulated time response of the temperature change during the first $50 \mu$ s of the heating cycle.

Figure 3.13 Simulated temperature distribution (a) on the cross-section (b) along xaxis across the microring

Figure 3.14 Design of ring-shape micro-heater

Figure 3.15 Simulated temperature distributions of (a) the whole device (b) $x-z$ plane cross-section at the geometry center of the microring (c) along the microring. All temperatures in Kelvin. The coordinate is shown in (a)

Figure 3.16 (a) Simulated time response of the temperature change of the microring with ring-shape micro-heater;(b) Normalized simulated time response of the temperature change with ring-shape micro-heater.

Figure 3.17 Time response of average microring temperature change with the two micro-heater designs

Figure 3.18 Simulated effective index versus temperature for (a) TE mode (b) TM mode.

Figure 4.1 Fabrication process flow of the tunable microring resonator device

Figure 4.2 SEM pictures of (a) patterned HSQ resist at gap between microring and straight waveguide (b) microring resonator device after silicon etch (c) microring resonator device with $\mathrm{SiO}_{2}$ cladding

Figure 4.3. Top-view microscopic image of micro-heater on top of $\mathrm{Si}$ based

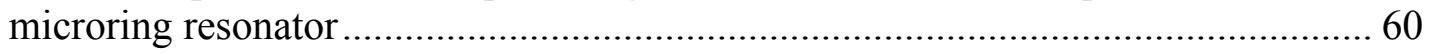

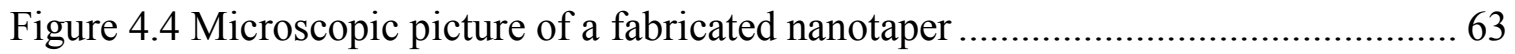

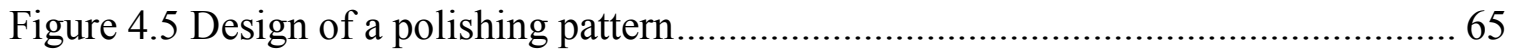

Figure 4.6 Microscopic picture of electrode pad connected to a micro-heater feedline. The dark lines are silicon waveguide which is under the metal microheater and feedline/pad layer separated by the upper cladding of $1.4 \mu \mathrm{m}$.

Figure 5.1 Experimental setup block diagram for device characterization. Obj.objective lense, TF -tapered fiber, ASE- amplified spontaneous emission source, OSA-optical spectrum analyzer, PC-polarization controller, TLwavelength tunable laser, OM-optical meter, PD-photodiode.

Figure 5.2 Pictures of the characterization setup 
Figure 5.3 Measured drop port transmission spectra of a fabricated tunable microring resonator at room temperature ofC22with TE and TM input polarization. Inset shows the resonance spectrum of peak around $1555 \mathrm{~nm}$ with higher resolution.

Figure 5.4 Measured group indices (dots) for (a) TE and (b) TM polarization compared with simulated calculations based on FDTD (solid), beampropagation (dashed).

Figure 5.5 Measured quality factor (dots) for (a) TE and (b) TM polarization and compared with simulated (solid)

Figure 5.6 Normalized drop port transmitted power spectra taken at different sample temperature with TE input polarization. Legends shows the sample temperature when spectrum was measured.

Figure 5.7 Measured resonance shift versus temperature change of five TE resonance peaks when tuning the sample temperature.

Figure 5.8 Normalized drop port TE transmitted power spectra taken at various heating currents. Legend shows the constant currents applied to the microheater when the spectrum was measured.

Figure 5.9 Resonance shift versus (a) micro-heater current applied (b) current square.

Figure 5.10 Measured output power transients for processes of (a) tuning to $\lambda_{\mathrm{t}}(\mathrm{b})$ detuning from $\lambda_{\mathrm{t}}$ (c) tuning to $\lambda_{0}(\mathrm{~d})$ detuning from $\lambda_{0}$, when tuning the resonance with wavelength $\lambda_{0}=1573.76 \mathrm{~nm}$. Different curves in each plot shows the measurements with different wavelength shift $\left(\Delta \lambda=\lambda_{\mathrm{t}}-\lambda_{0}\right)$ of $0.2,0.4$, $0.8,1.6,3.2$ and $6.4 \mathrm{~nm}$ Each curve was normalized related to its maximum.

Figure 5.11 Simulated output power transients for processes of (a) tuning to $\lambda_{t}(b)$ detuning from $\lambda_{\mathrm{t}}$ (c) tuning to $\lambda_{0}(\mathrm{~d})$ detuning from $\lambda_{0}$, when tuning the resonance with wavelength $\lambda_{0}=1573.76 \mathrm{~nm}$. Different curves in each plot shows the measurements with different wavelength shift $\left(\Delta \lambda=\lambda_{\mathrm{t}}-\lambda_{0}\right)$ of $0.2,0.4$, $0.8,1.6,3.2$ and $6.4 \mathrm{~nm}$ Each curve was normalized related to its maximum.

Figure 5.12 Plots of tuning rise delay times of room-temperature resonance peaks at (a) $1573.76 \mathrm{~nm}$ (Peak A), (b) $1592.65 \mathrm{~nm}$ (Peak B) and (c) $1611.80 \mathrm{~nm}$ (Peak C) as well as plots of fall delay times of the peaks (d), (e) and (f), respectively. The curves are simulated results and the dots are measured values. The insets are the corresponding peak spectra. Note the different time scale between rise and fall plots. 
Figure 6.1 Layout diagrams of the (a) $1 \times 4$ and (b) $4 \times 4$ wavelength reconfigurable photonic switches based on thermally tunable microring resonators on SOI substrate.

Figure 6.2 Picture of the TEC temperature-regulated $4 \times 4$ switch device and PCB assembly

Figure 6.3 Output spectrum of the device showing wavelength routing of 1562.43 , $1562.83,1563.23$, and $1563.63 \mathrm{~nm}$ to output ports $4,3,1$, and 2 , respectively 95

Figure 6.4 One switching configuration of the $4 \times 4$ photonic switch with ${ }_{1}, \lambda_{2}, \lambda_{3}$, and $\lambda_{4}$ routed from input ports $(1,2,3$, and 4$)$ to output ports $(4,2,3$ and 1$)$, respectively.

Figure 6.5 Transmission spectrum of a microring resonator with various heating currents sent to an adjacent micro-heater.

Figure 6.6 (a) Experimental setup of the tunable laser (b) top view microscopic

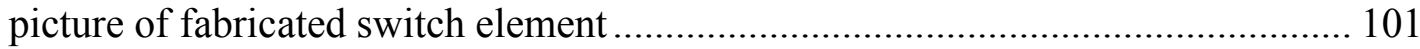

Figure 6.7 (a) Transmission spectrum of the filter using unpolarized ASE source. Lasing spectra of: (b) TE mode; (c) TM mode; and (d) simultaneous lasing of both TE and TM modes. Insets show corresponding top view infrared images of the ring area during lasing.

Figure 6.8 Laser output power versus the EDF pumping bias current. Inset shows the EDF gain at $1550 \mathrm{~nm}$ for different input power at pumping current from 100 $\mathrm{mA}$ to $400 \mathrm{~mA}$

Figure 6.9 (a) Filter spectra and (b) lasing spectra at applied heating current of 0, 1, 3 and $5 \mathrm{~mA}$ (c) filter and lasing peak wavelength shift versus applied heating power. 


\section{INTRODUCTION}

\subsection{Transition from Electronics to Photonics in High-speed Communications}

Now, we are in the information age enabled by the development of the internet. The evolution of the internet and pervasive computing creates massive bandwidth demand for data communications. As industries move to higher and higher bandwidths, electronic communication links are approaching the fundamental distance $\times$ bandwidth limitation governed by the physical loss mechanisms (a function of distance and bandwidth) and noise levels. For example, the bandwidth for today's high-speed copper interconnects in computing systems is roughly $3 \mathrm{GHz}$ within a $1 \mathrm{~m}$ distance. Most experts feel that copper interconnect limits will be reached in the $10-40 \mathrm{GHz}$ range for $\mathrm{PC}$ board materials within $1 \mathrm{~m}$ distances. Meaning that these interconnect limits will be reached in only a few generations. [1]. Moving close to a fundamental limit usually involves ever-increasing costs. To ease cost pressure, many industries have chosen to find alternative technology platforms that do not suffer from the same physical limitations. For many industries with long communication distances such as the long-haul industry and the storage area network industry; this choice has been to switch to photonics [1]. As the communication bandwidth demand gets higher and higher, applications with shorter and shorter communication distances are expected to migrate to photonics.

In optical communication systems, large communication bandwidth can be

achieved by wavelength-division multiplexing (WDM) technology. WDM is a technology which multiplexes a number of optical carrier signals onto a single optical fiber by using different wavelengths of laser light to carry different signals. This allows 
for a multiplication in capacity, in addition to enabling bidirectional communications over one strand of fiber. The S-, C-, L- spectral bands are commonly used for WDM systems because of low optical loss in standard optical fiber [31]. The band covers the wavelength range of $1460-1625 \mathrm{~nm}$ ( $\sim 20 \mathrm{THz}$ optical bandwidth), allowing an upper limit total data rate of $20 \mathrm{Tbps}$ for a single piece of fiber. With zero-water-peak fiber becoming commercially available recently [32], the wavelength range can be expanded even wider from $\sim 1260 \mathrm{~nm}$ to $1625 \mathrm{~nm}(\sim 56 \mathrm{THz})$.Dense wavelength-division multiplexing (DWDM) uses the same communication band, but with denser channel spacing to achieve high bandwidth efficiency. With optical fibers, information can be transmitted thousands of kilometers away with optical amplification. Compare to electrical communication systems, optical communication systems have a huge advantage on the distance $\times$ bandwidth product.

The transition of communication systems from electronics to photonics depends strongly on the cost of the systems. Conventional photonic components usually have much higher cost compared to electronic components. Some of them are 10 to 100 times more expensive than their electronic equivalents. Lowering the cost of the optical solutions is essential for the transition timeframe. The advent of an optical replacement solution with suitable cost during this transition will enable earlier conversion. The absence of such a suitable optical replacement will delay conversion until the cost of electrical components become prohibitive. 


\subsection{Silicon-based Integrated Photonics}

Silicon technology is promising as a means to lower the cost of the optical communication systems. Over the last fifty years, the rapid development of the electronics industry based on the manufacturing of silicon electronic devices has already shown enormous performance improvement and cost reduction. Many improvements have been introduced in the manufacturing of electronic devices to keep pace with the steady evolution dictated by Moore's law; which states that the number of transistors on an integrated circuit would double approximately every 2 years [33]. By applying silicon technology in photonics, we envision similar reduction in cost and improvements in performance.

Most integrated photonics device now in market are based on III-V semiconductors or planar lightwave circuits (PLCs). With low refractive index contrast between waveguide and cladding, PLC-based device are usually large, not good for high integration. And compared with III-V semiconductors, silicon offers the advantages of mature fabrication technology and potential monolithic integration with complementary metal-oxide-semiconductor (CMOS) devices, which would enable mass production of photonics devices with low cost. In addition, silicon-on-insulator (SOI) wafers widely used in electronic integrated circuits also provide an ideal platform for optical and optoelectronic integrated circuits. The underlying thermal oxide provides simultaneous high-quality optical and electrical isolation. Also, silicon-based photonics provides a golden opportunity to introduce and marry photonics with electronics. This new functionality is not only breaking the bandwidth barrier, but is also offering design freedom, small footprint with minimal latency penalty, and power efficiency. With its 
prospect of breaking the bandwidth bottleneck while extending a massive, low-cost electronics manufacturing platform into the photonics domain, silicon-based integrated photonics has been the subject of much research in recent years; because of the clear advantages of the photonic technology on the silicon platform.. Significant advancements had been made in silicon-based integrated photonics recently. Monolithic waveguideintegrated Ge photodetector offers over $40 \mathrm{GHz}$ bandwidth, with a quantum efficiency of over $90 \%$ at the $1550 \mathrm{~nm}$ band [2]. Optical modulators have been reduced to a small size with very low power dissipation [3]. Silicon microring resonators provides wavelength filtering with free spectral range (FSR) of over 60nm. [4].

\subsection{Integrated Photonics towards DWDM Applications}

There is a great opportunity for integrated photonics in DWDM applications to enable reaching the limits of bandwidth efficiency, by providing sophisticated wavelength filtering in which conventional optical analogues prove difficult or impractical to realize. Particularly, when narrow pass-band and high wavelength channel count are required, conventional technologies become less economical. Furthermore, the introduction of more complex functionality including wide tuning range and multiple channel drops make realization in conventional bulk technologies more and more challenging. Complexity, dense integration and low power are the advantages for integrated photonics.

One of the key building blocks for the implementation of next-generation flexible and cost-effective WDM networks is reconfigurable optical add/drop multiplexer 
(ROADM) [34].The present WDM networks use mainly fixed optical add/drop multiplexers (OADMs); in which the number of wavelengths in the network and their service patterns are fixed and predetermined. This imposes a significant limitation on the service providers in the management of their optical networks. The lack of flexibility and slow service provisioning are the main challenges of building the dynamically provisioned network of future. Therefore, the role of development and integration of ROADMs into the present WDM networks is of critical value. The switching or reconfiguration functions of a ROADM are commonly achieved using switching technologies including MEMS [36, 39], PLC [53, 54], liquid crystal [55] and wavelengthtunable filters [68]. MEMS devices are based on complex architectures and usually need sophisticated fabrication processes. PLC-based ROADM normally use array waveguide gratings (AWGs) to assign lightwaves with different wavelengths into channels, and need to use a separate switch on each channel. Liquid crystal approaches rely on conventional optical gratings for wavelength demultiplexing which make the devices bulky. Compared to the other approaches, the wavelength-tunable filter is a promising technology due to its simple architecture and good scalability for high port count DWDM applications.

Other than ROADM application, integrated wavelength-tunable filter can be applied in other applications in DWDM optical network. Recent progress in DWDM technology has called for multi-wavelength light sources. The multi-wavelength transmitters for the WDM system are currently built with multiple discrete lasers [86]. However, combining discrete lasers for a WDM light source suffers from the high packaging cost and the burden of multiple alignments between laser diodes and optical fibers. Integration of lasers into a multi-wavelength laser array has been presented as a 
promising approach to reduce the cost per wavelength so that the cost of packaging and of the required pigtail optics can be shared by a large number of wavelengths $[87,88]$. By multiplexing wavelength-tunable filters, multi-wavelength laser sources could be realized using a common gain media.[89]

WDM networks have been limited by the requirement of exact wavelength registration throughout the network, which imposes extremely tight and costly wavelength tolerances on the sources. However, the application of wavelength-tunable filters can relax these tolerances and make the system more robust. [90, 91] Using wavelength-tunable filters, receivers can be tuned to the channel wavelength of the sources of interest and locked to that wavelength using feedback while allowing changes of source wavelength. For DWDW systems while multi-wavelength sources are used, integrated wavelength-tunable filters will be promising for cost-efficient simultaneously multi-wavelength tracking.

\subsection{Choosing Silicon for Wavelength Tuning}

Wavelength tuning for integrated photonic filters can be realized through several mechanisms, such as thermooptic induced index change [37, 38, 69-72], electrooptic effect $[73,74]$, carrier induced index changes $[3,20,75]$, and dielectric perturbation by MEMS [26].

For many applications in DWDM networks such as ROADM, wide tunable range is desired while the tuning speed is not critical; with millisecond time scale sufficient for operations. Thus large index change is needed. Electro-optic effect is fast, however, it 
cannot generate a large index change with practical voltage applied. Carrier induced index change by carrier injection [3] or two-photon absorption [20] is relatively small and always accompanies absorption. However, this mechanism can be used for modulation due to its high speed. MEMS approach usually requires sophisticated fabrication processes. Hence, we choose thermooptic tuning for this application.

Materials with high thermooptic coefficient (on the order of $10^{-4} / \mathrm{K}$ ) include semiconductors such as $\mathrm{Si}, \mathrm{InP}$, and polymers. Silicon is determined to be more suitable for this application as it readily forms strongly-confined, high index contrast waveguides using $\mathrm{SiO}_{2}$ as cladding. Polymers [76] generally have a low refractive index, and usually prove difficult to form large index contrast system desired to achieve large FSR and high integration. Meanwhile III-V semiconductors such as InP do not have readily available low-index under cladding materials needed for low-loss and strong confinement.

Reconfigurable wide wavelength-tuning range $(9.4 \mathrm{~nm})$ was demonstrated with polymer-based devices [71] by thermooptic index tuning prior to this work. However, the device has a relatively large resonance linewidth, (about $2 \mathrm{~nm}$ ) limited by its relatively low index contrast, and is thus not suitable for DWDM applications. A relatively narrow tunable range $(\sim 1 \mathrm{~nm})$ was achieved by carrier injection on III-V devices [75]. Using thermooptic tuning on $\mathrm{Si}_{3} \mathrm{~N}_{4}$ device with $\mathrm{SiO}_{2}$ cladding, a wavelength tunable range of 3$4 \mathrm{~nm}$ was achieved $[68,72]$. Initial study by the author in 2006 had shown thermooptic tuning of $0.11 \mathrm{~nm} /{ }^{\circ} \mathrm{C}$ on a silicon-based integrated filter [43]. Thus, much wider tunable range is expected by implementing thermooptic tuning on silicon-based integrated filter. Recent advances on silicon-based wavelength-tunable filters demonstrated an ultra-wide tunable range of $20 \mathrm{~nm}[38]$. 


\subsection{Objective of This Work}

The objective of this work is to design a silicon-based integrated wavelengthtunable filter/switch feasible for DWDM applications. To achieve both bandwidth and cost efficiency, some key specifications of the tunable filter are desired:

1. Large free spectral range (FSR) and relatively small filter linewidth to allow a large number of channels

2. Large wavelength tunable range to allow a large number of accessible channels for filter

3. Compatible with common semiconductor fabrication facilities

4. Good scalability and compact footprint for dense integration

5. Low power consumption

\subsection{Organization of the Dissertation}

In this dissertation, we have investigated the design, simulation and characterization of a silicon-based wavelength tunable microring resonator and its applications.

Chap. II covers the main aspects of microring resonator theory including the wavelength tuning mechanism. In Chap. III, detailed design and theoretical analysis of the device are explained, including the optical and thermal design. Chap. IV described the fabrication process flow of the device. The characterization of the device is covered in Chap. V. The characterization results are compared with the theoretical model and 
followed by a discussion in the chapter. In Chap. VI, we demonstrated two applications which suggest the feasibility of the device towards DWDM applications. Finally, the dissertation concludes in Chap. VII. 


\section{THEORY OF MICRORING RESONATOR}

\subsection{Introduction of Microring Resonator}

The microring resonator is a wavelength-selective integrated photonic device. The most common application for the microring resonator is optical filtering. When used as an optical filter, the microring resonator provides great design flexibility to meet desired filter specifications. In this work, it will be configured as optical add/drop-type filter for DWDM applications.

Essentially, an optical resonator is an optical cavity that resonates at some specific wavelengths which are defined as resonance wavelengths. When properly excited, light waves can propagate in a back-and-forth or circulating manner in the optical resonator with minimum attenuation. The optical resonator can be in the form of two mirrors facing each other, microdisk, microring, microsphere or other type of cavities. Among the optical resonators, microring resonators don't require facets or gratings for optical feedback and are thus particularly suited for monolithic integration with other components.

Microring resonators consists of a waveguide in a close loop, commonly in the shape of a ring or race-track. When placing the loop within close proximity of an input waveguide, light can be coupled into the cavity via evanescent field, and can propagate around the periphery of the cavity. Resonance will take place because of the constructive interference for light whose phase change after each full trip around the closed loop is an integer multiple of $2 \pi$, i.e., in phase with the incoming light. Light not meeting this resonance condition will be transmitted through the input waveguide. 
Microring resonator is now considered as one of the most important building block of integrated photonics and has gained widespread interest over the last decade. Various functions have been realized based on the microring resonator configuration, including optical filters [5], optical modulators [3], add-drop multiplexers [6], optical sensors $[7,8]$, optical dispersion compensators, $[9,10]$ and even laser source [11].

Silicon microring resonators have been an active research area in favor of recent advances in the fabrication technologies. With high refractive index, silicon provides tight optical confinement necessary for high-density photonic integration and nonlinear optics. Extremely compact microring resonators $[43,4]$ have been demonstrated. Due the compact size, larger FSR of $47 \mathrm{~nm}(5.9 \mathrm{THz})$ and $62.5 \mathrm{~nm}(7.8 \mathrm{THz})$ at wavelengths around $1550 \mathrm{~nm}$ were realized, which allowed a large number of channels to be supported, enabling DWDM.

\subsection{Model of Microring Resonator with Standard Configuration}

The theoretical analysis of microring resonators has been well established and studied using the oscillator model [56] and the coupling model [57]. In this work, we use the coupling model. Section 2.2 and 2.3 in this work follows derivations in ref [58] with a different nomination system.

The standard add/drop configuration for a microring resonator consists of two straight waveguides and a ring as described in Figure 2.1. The straight waveguides, also known as the bus or the port waveguides, are coupled either by directional couplers through the evanescent field or by multimode interference (MMI) couplers to the 
microring. In order to numerically analyze the model, some assumptions are made to simplify the case. The assumptions include:

1. There only exists single unidirectional mode of the resonator

2. Only one polarization is considered

3. The coupling between bus waveguide and the microring is lossless

4. The losses of various kinds distribute evenly along the propagation path in the microring resonator

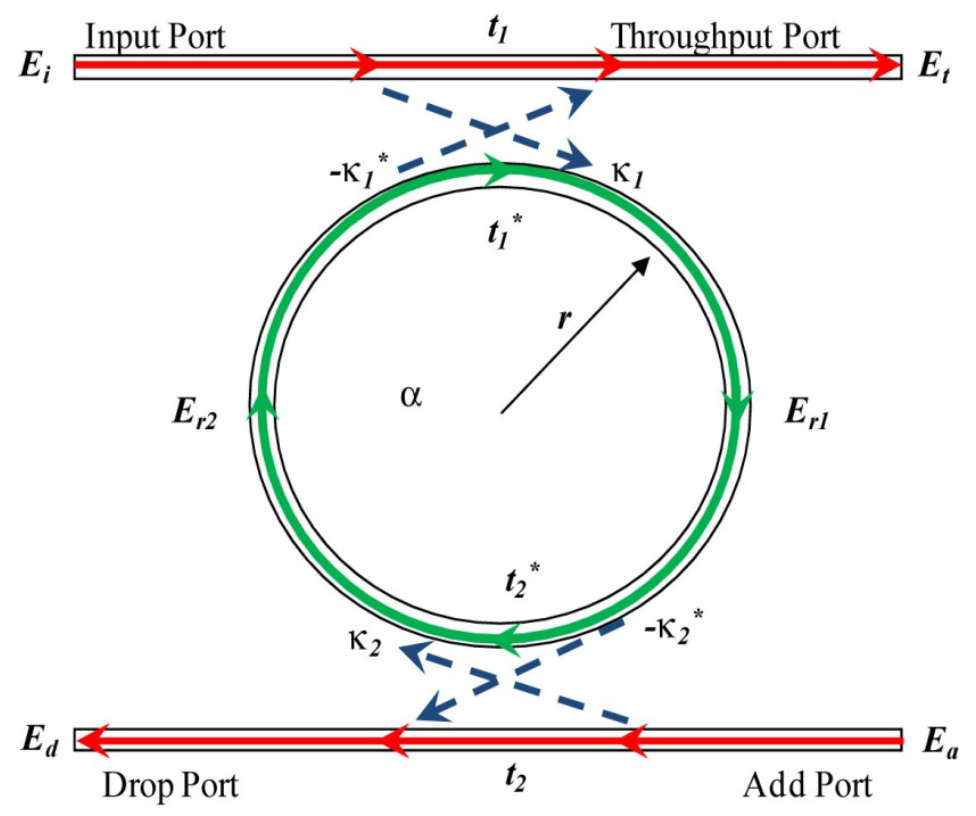

Figure 2.1 Model of a standard add/drop type microring resonator

As shown in Figure 2.1, $E_{i}, E_{t}, E_{d}$ and $E_{a}$ are the normalized complex mode amplitudes at the input port, throughput port, drop port and add port, respectively. And $t_{1}$, $t_{2}$ are the transmission coefficients, and $\kappa_{1}, \kappa_{2}$ are coupling coefficients at the two coupling regions. The microring has a radius of $r$ with inner circulation factor of the microring defined as $\alpha$. For zero internal loss $\alpha=1$. The $*$ denotes the conjugated 
complex value. The couplers under consideration are reciprocal, thus the following relationship between the coupler parameters are valid:

$$
\begin{aligned}
& \left|\kappa_{1}^{2}\right|+\left|t_{1}^{2}\right|=1 \\
& \left|\kappa_{2}^{2}\right|+\left|t_{2}^{2}\right|=1
\end{aligned}
$$

At the upper coupler shown in Figure 2.1, the interaction can be described by the matrix:

$$
\left(\begin{array}{c}
E_{t} \\
E_{r 1}
\end{array}\right)=\left(\begin{array}{cc}
t_{1} & -\kappa_{1}^{*} \\
\kappa_{1} & t_{1}^{*}
\end{array}\right)\left(\begin{array}{c}
E_{i} \\
E_{r 2}
\end{array}\right)
$$

While at the lower coupler, we obtain:

$$
\left(\begin{array}{c}
E_{r 2} /\left(\alpha^{\frac{1}{2}} \cdot e^{\frac{j \varphi}{2}}\right) \\
E_{d}
\end{array}\right)=\left(\begin{array}{cc}
t_{2}^{*} & \kappa_{2} \\
-\kappa_{2}^{*} & t_{2}
\end{array}\right)\left(\begin{array}{c}
\alpha^{1 / 2} \cdot e^{j \varphi / 2} E_{r 1} \\
E_{a}
\end{array}\right)
$$

where $\varphi$ is the phase change of the mode propagation:

$$
\varphi=\frac{2 \pi \cdot\left(n_{g} \cdot L\right)}{\lambda}=\frac{2 \pi \cdot\left(n_{g} \cdot 2 \pi r\right)}{\lambda}=4 \pi^{2} n_{g} \frac{r}{\lambda}
$$

$L$ is the perimeter of the ring and $\lambda$ is the wavelength of the light beam. The index $n_{g} \equiv \partial \beta / \partial k$ denotes the effective group index of the propagation mode in a guide-wave system. We simplified it as "group index" in this work. It is different from the effective mode index $n_{\text {eff }} \equiv \beta / k$ which will be used later in this work and is simplified as "effective index". $\beta$ is the propagation coefficient and $k \equiv 2 \pi / \lambda$ is the vacuum wavevector.

To further simplify the model, $E_{i}$ is chosen to be 1 and we assume there is no light from the add port, i.e. $E_{a}=0$. Equations (2.3) and (2.4) can be simplified as following:

$$
\begin{aligned}
& E_{t}=t_{1}-E_{r 2} \cdot \kappa_{1}^{*} \\
& E_{r 1}=\kappa_{1}+E_{r 2} \cdot t_{1}^{*}
\end{aligned}
$$




$$
\begin{gathered}
E_{r 2}=t_{2}^{*} \cdot \alpha \cdot e^{i \varphi} E_{r 1} \\
E_{d}=-\kappa_{2}^{*} \cdot \alpha^{1 / 2} \cdot e^{j \varphi / 2} E_{r 1}
\end{gathered}
$$

By solving the mode amplitudes from the equations above, the mode amplitudes are given by

$$
\begin{gathered}
E_{r 1}=\frac{\kappa_{1}}{1-t_{1}^{*} t_{2}^{*} \alpha e^{i \varphi}} \\
E_{r 2}=\frac{\kappa_{1} t_{2}^{*} \alpha e^{i \varphi}}{1-t_{1}^{*} t_{2}^{*} \alpha e^{i \varphi}} \\
E_{d}=\frac{-\kappa_{1} \kappa_{2}^{*} \alpha^{1 / 2} e^{j \varphi / 2}}{1-t_{1}^{*} t_{2}^{*} \alpha e^{i \varphi}} \\
E_{t}=t_{1}-\frac{\kappa_{1}^{*} \kappa_{1} t_{2}^{*} \alpha e^{i \varphi}}{1-t_{1}^{*} t_{2}^{*} \alpha e^{i \varphi}}=\frac{t_{1}-t_{2}^{*} \alpha e^{i \varphi}}{1-t_{1}^{*} t_{2}^{*} \alpha e^{i \varphi}}
\end{gathered}
$$

As the complex mode amplitudes are normalized, their squared magnitude corresponds to the modal power. The circulating power $P_{r}$, the drop port power $P_{d}$ and the through port power $P_{t}$ are given by

$$
\begin{gathered}
P_{r}=\left|E_{r 2}\right|^{2}=\frac{\left(1-\left|t_{1}\right|^{2}\right)\left|t_{2}\right|^{2} \alpha^{2}}{1+\left|t_{1}\right|^{2}\left|t_{2}\right|^{2} \alpha^{2}-2\left|t_{1}\right|\left|t_{2}\right| \alpha \cdot \cos \left(\varphi-\theta_{1}-\theta_{2}\right)} \\
P_{d}=\left|E_{d}\right|^{2}=\frac{\left(1-\left|t_{1}\right|^{2}\right)\left(1-\left|t_{2}\right|^{2}\right) \alpha}{1+\left|t_{1}\right|^{2}\left|t_{2}\right|^{2} \alpha^{2}-2\left|t_{1}\right|\left|t_{2}\right| \alpha \cdot \cos \left(\varphi-\theta_{1}-\theta_{2}\right)} \\
P_{t}=\left|E_{t}\right|^{2}=\frac{\left|t_{1}\right|^{2}+\left|t_{2}\right|^{2} \alpha^{2}-2\left|t_{1}\right|\left|t_{2}\right| \alpha \cdot \cos \left(\varphi-\theta_{1}-\theta_{2}\right)}{1+\left|t_{1}\right|^{2}\left|t_{2}\right|^{2} \alpha^{2}-2\left|t_{1}\right|\left|t_{2}\right| \alpha \cdot \cos \left(\varphi-\theta_{1}-\theta_{2}\right)}
\end{gathered}
$$

where $t_{1}=\left|t_{1}\right| \exp \left(i \theta_{1}\right)$ and $t_{2}=\left|t_{2}\right| \exp \left(i \theta_{2}\right), \theta_{1}, \theta_{2}$ representing the phase changes of the couplers.

On resonance, $\left(\varphi-\theta_{1}-\theta_{2}\right)=2 \pi m$, where $m$ is an integer, the following are obtained: 


$$
\begin{gathered}
P_{\text {r-resonance }}=\frac{\left(1-\left|t_{1}\right|^{2}\right)\left|t_{2}\right|^{2} \alpha^{2}}{\left(1-\alpha\left|t_{1} t_{2}\right|\right)^{2}} \\
P_{d-\text { resonance }}=\frac{\left(1-\left|t_{1}\right|^{2}\right)\left(1-\left|t_{2}\right|^{2}\right) \alpha}{\left(1-\alpha\left|t_{1} t_{2}\right|\right)^{2}} \\
P_{t-\text { resonance }}=\frac{\left(\left|t_{1}\right|-\left|t_{2}\right| \alpha\right)^{2}}{\left(1-\alpha\left|t_{1} t_{2}\right|\right)^{2}}
\end{gathered}
$$

By observing Equation (2.18), full transfer of power from the input port to the drop port, i.e., $P_{\text {d-resonance }}=1$, occurs when both of the following conditions are satisfied: $\alpha=1$ (negligible internal losses) and $\left|t_{1}\right|=\left|t_{2}\right|$ (symmetric coupling). In this case, the throughput port power will be zero. In reality, the value $\alpha=1$ cannot be satisfied without gain incorporated to the microring. For a passive microring resonator (without any gain within the microring), $\alpha$ is always smaller than 1. From (2.19), to achieve zero power at the throughput port $\left(P_{t \text {-resonance }}=0\right)$, the relation of $\alpha=\left|t_{1} / t_{2}\right|$ must be satisfied. Thus, the full transfer of power to the drop port in real case, which we called "critical coupling" condition, can only be satisfied in microring resonators with asymmetric coupling.

\subsection{Parameters of Microring Resonator}

Microring resonator filters can be described by some points of merit which are also generally used to describe optical filters. In this part of the chapter, we will introduce some important parameters for the microring resonator based on the derived model in the last part. 


\subsubsection{Free spectral range (FSR)}

One important parameter for microring resonators is the free spectral range (FSR), which is the distance between two adjacent resonance peaks. For a microring resonator, when on resonance, $2 \pi m=L \cdot \beta$ will be satisfied, where $m$ is an integer, $L$ is the perimeter of the ring and $\beta$ is the propagation coefficient. Group index, as defined previously can be written as $n_{g}=\partial \beta / \partial k=\partial \beta / \partial\left(\frac{2 \pi}{\lambda}\right)=\frac{\partial \beta}{\partial \lambda \cdot\left(-\frac{2 \pi}{\lambda^{2}}\right)}$. Neglecting the wavelength dependence of group index $n_{\mathrm{g}}$,

$$
F S R=\Delta \lambda=\frac{\Delta \beta}{\frac{\partial \beta}{\partial \lambda}}=\frac{-\frac{2 \pi}{L}}{\frac{\partial \beta}{\partial \lambda}}=\frac{-\frac{2 \pi}{L}}{-n_{g} \cdot \frac{2 \pi}{\lambda^{2}}}=\frac{\lambda^{2}}{n_{g} \cdot L} \approx \frac{\lambda^{2}}{n_{g} \cdot(2 \pi r)}
$$

As we can see, FSR is inversely proportional to the radius of the ring. In many applications such as communications, a large FSR is usually desired to achieve a higher communication bandwidth. Thus, a smaller radius would be beneficial.

\subsubsection{Full width at half maximum (FWHM)}

The next parameter of importance is the resonance width which is defined as the full width at half maximum (FWHM) or $3 \mathrm{~dB}$ bandwidth of the resonance lineshape. Using the expression for the drop port (2.15), we obtain

$$
\frac{\left(1-\left|t_{1}\right|^{2}\right)\left(1-\left|t_{2}\right|^{2}\right) \alpha}{1+\left|t_{1}\right|^{2}\left|t_{2}\right|^{2} \alpha^{2}-2\left|t_{1}\right|\left|t_{2}\right| \alpha \cdot \cos \left(\varphi-\theta_{1}-\theta_{2}\right)}=\frac{1}{2}\left(\frac{\left(1-\left|t_{1}\right|^{2}\right)\left(1-\left|t_{2}\right|^{2}\right) \alpha}{1+\left|t_{1}\right|^{2}\left|t_{2}\right|^{2} \alpha^{2}-2\left|t_{1}\right|\left|t_{2}\right| \alpha}\right)
$$

Let $\phi=\varphi-\theta_{1}-\theta_{2}$, we have

$$
1+\left|t_{1}\right|^{2}\left|t_{2}\right|^{2} \alpha^{2}-2\left|t_{1}\right|\left|t_{2}\right| \alpha \cdot \cos (\phi)=2\left(1+\left|t_{1}\right|^{2}\left|t_{2}\right|^{2} \alpha^{2}-2\left|t_{1}\right|\left|t_{2}\right| \alpha\right)
$$


For small $\phi$, using the first 2 items of Taylor expansion of Euler formula,

$$
\cos \phi=1-\frac{\phi^{2}}{2}
$$

Therefore

$$
\phi^{2}=\frac{\left(1-\left|t_{1}\right|\left|t_{2}\right| \alpha\right)^{2}}{\left|t_{1}\right|\left|t_{2}\right| \alpha}
$$

If the loss of the ring is negligible and the coupling of the two coupler is symmetric $\left(t=t_{1}=t_{2}\right)$,

$$
\phi=\sqrt{\frac{\left(1-t^{2}\right)^{2}}{t^{2}}}=\frac{1-t^{2}}{t}
$$

In the wavelength domain, compared to the phase change of $2 \pi$ for FSR, we have $F W H M / F S R=2 \cdot \phi / 2 \pi$

$$
F W H M=2 \cdot \frac{\phi}{2 \pi} \cdot F S R=\frac{\lambda^{2}}{\pi n_{g} L} \frac{1-t^{2}}{t}
$$

Assuming weak coupling $(\kappa \ll 1)$, note that $\kappa^{2}+t^{2}=1$, we have $t \approx 1$, then

$$
F W H M=2 \cdot \frac{\phi}{2 \pi} \cdot F S R=\frac{\lambda^{2} \cdot \kappa^{2}}{\pi n_{g} L}
$$

FWHW or $3 \mathrm{~dB}$ bandwidth is widely used in describing the spectral width of an optical source.

\subsubsection{Finesse $(F)$}

The parameter finesse (F) is defined to be the ratio of the FSR and FWHM: 


$$
F=\frac{F S R}{F W H M}=\pi \frac{t}{1-t^{2}} \approx \frac{\pi}{\kappa^{2}}(t \approx 1)
$$

With the same FSR, a resonator with higher $F$ shows sharper peak.

\subsubsection{Quality factor (Q)}

For resonators, quality factor $(Q)$ is an essential parameter. There are two separate definitions for quality factor which are equivalent for high $\mathrm{Q}$ resonators [59]. Generally, $Q$ is defined in terms of the ratio of the peak energy stored in the resonator to that of the energy being lost in one cycle such that:

$$
Q=2 \pi \frac{E_{\text {stored per cycle }}}{E_{\text {dissipated per cycle }}}
$$

The factor $2 \pi$ is used to keep this definition of $Q$ (for high values of $Q$ ) with the second definition:

$$
Q=\frac{f_{\text {resonance }}}{\Delta f}=\frac{\lambda}{\mathrm{FWHM}}=\frac{\pi n_{g} L}{\lambda} \frac{t}{1-t^{2}}=\frac{n_{g} L}{\lambda} F
$$

The quality factor $Q$ of a resonator is a measure of sharpness of the resonance. Resonators having higher $\mathrm{Q}$ factors resonate with greater amplitudes (at the resonant frequency) but have a smaller range of frequencies around that frequency for which they resonate. Thus, a high $\mathrm{Q}$ resonator has higher selectivity; it would do a better job of filtering out undesired signals that lay nearby on the spectrum. From Equation (2.30) it is proportional to the Finesse $(F)$. 


\subsubsection{Intensity enhancement factor (B)}

The intensity in the microring resonator can be much higher than that in the bus waveguides, as the traveling light in the microring resonator interferes constructively at the resonance with the input light and thus the amplitude builds up. Other than the intensity, the field has a phase-shift of an integer time of $2 \pi$ each cycle. With the configuration as in Figure 2.1, the intensity enhancement factor $(B)$ can be described as

$$
B=\left|\frac{E_{r 1}}{E_{i}}\right|^{2}=\left|\frac{\kappa_{1}}{1-t_{1}^{*} t_{2}^{*} \alpha e^{i \varphi}}\right|^{2}
$$

On resonance,

$$
B=\left|\frac{\kappa_{1}}{1-t_{1}^{*} t_{2}^{*} \alpha}\right|^{2}
$$

Assuming lossless $(\alpha=1)$ resonator and symmetric and weak coupling $\left(\kappa_{1}=\kappa_{2}=\right.$ $\kappa \ll 1)$, B can be simplified as

$$
B=\frac{1}{\kappa^{2}}=\frac{F}{\pi}
$$

Microring resonators can be used for nonlinear optical device because of the much higher intensity achievable in the microring compared to the bus ports.

\subsubsection{Extinction ratio $\left(r_{e}\right)$}

The parameter of extinction ratio $\left(r_{e}\right)$ for the microring resonator is the ratio between the on-resonance drop port power and off-resonance drop port power, usually in $\mathrm{dB}$ scale. High extinction ratio is desired to achieve low error bit rate (EBR) in communication. 


\subsection{Tuning of Microring Resonator}

The optical performance of a resonator can be tuned using different approaches. With the microring resonator model established previously, the tuning can be generally done by adjusting three major parameters: the inner circulation factor $(\alpha)$, the power coupling coefficient $\left(\kappa^{2}\right)$ and the group index $\left(n_{g}\right)$. The inner circulation factor $(\alpha)$ can be tuned by electro-absorption [12], gain trimming [13], and metal absorption [14]. The tuning of power coupling coefficients $\left(\kappa^{2}\right)$ are usually applied in optical bandwidth tuning [24] by using Mach-Zehnder interferometer (MZI) [25], micro-electro-mechanical system (MEMS) [26-29] and micro-fluidic approaches [30]. Wavelength tuning is usually done through changing group index $\left(n_{g}\right)$ by thermooptic effect [15-17], electro-optical effect [18], and carrier injection [19]. Wavelength tuning can also be achieved by adjusting cavity length by strain [21-23].

Assuming the two couplers are symmetric $\left(\kappa=\kappa_{1}=\kappa_{2}, t=t_{1}=t_{2}\right)$ and the phase change due to coupling is negligible $\left(\phi=\varphi-\theta_{1}-\theta_{2} \approx \varphi\right)$, Equation 2.15 can be simplified as

$$
P_{d}=\frac{\left(1-|t|^{2}\right)^{2} \alpha}{1+|t|^{4} \alpha^{2}-2|t|^{2} \alpha \cdot \cos (\varphi)}
$$

And from Equation (2.5), at a wavelength $\lambda$ close to a resonance wavelength $\lambda_{0}$, we can obtain

$$
\cos (\varphi)=\cos \left(\frac{-4 \pi^{2} n_{g} r\left(\lambda-\lambda_{0}\right)}{\lambda_{0}{ }^{2}}\right)
$$


The optical spectral response can be simulated analytically, as demonstrated in ref $[58,40]$. To demonstrate the effects of different tuning approaches, the spectral responses while tuning a specific parameter were simulated analytically. By plugging Equation (2.35) into Equation (2.34), the drop port power $P_{d}$ can be written as a function of $\alpha, \kappa^{2}($ $\left.t^{2}=1-\kappa^{2}\right), n_{g}$ and $\lambda$. The drop port spectrum response $P_{d}$ versus $\lambda$ are simulated around a resonance using Equation (2.34) and (2.35). The resonance wavelength at $\sim 1556.01 \mathrm{~nm}$ was found around 1550nm using Equation (2.5) at resonance $(\varphi=2 \pi m)$.

Some typical parameters were assumed as follows: $\alpha=0.999, \kappa^{2}=0.02, \mathrm{r}=5 \mu \mathrm{m}$ and $n_{g}$ $=4.21$. Figure 2.2 (a), (b) and (c) illustrate the tuning of the simulated drop port spectrum response by altering $\alpha, n_{g}$ and $\kappa^{2}$, respectively. Figure 2.2(a) shows the spectrum change by adjusting $\alpha$ from 0.999 to 0.995 and 0.990 . The peak power dropped dramatically from 0.9 to 0.64 and 0.44 . Figure 2.2 (b) demonstrates the resonance wavelength change by controlling the group index. For a microring resonator with such a high $\mathrm{Q}$, at the original resonance wavelength, the drop port power can be tuned to about $20 \%$ of the original power with a minimal change of group index of 0.0003 . The group index change can be realized by changing only a couple of degrees in temperature due to the large thermooptic coefficient of silicon. The bandwidth tuning was shown in Figure 2.2(c) by altering $\kappa^{2}$. As also indicated in Equation (2.27), the resonance linewidth is proportional to the power coupling efficient. Apart from the bandwidth tuning, Figure 2.2(c) also shows the resonance power change when tuning the coupling ratio. This effect is due to the symmetry of the two couplers. As discussed previously, the critical coupling condition is satisfied when $\alpha=\left|t_{1} / t_{2}\right|$. For a symmetric configuration, the critical 
coupling cannot be achieved in reality because the ring cannot be lossless, i.e, $\alpha<1$. For the symmetric configuration with a fixed $\alpha$, the larger the coupling efficiency, the closer it will be to critical coupling and thus the higher the resonance power. To tune the bandwidth without tuning the resonance power can be achieved by individually tuning the coupling efficiency $[40,26]$ of the couplers to bring the resonator to critical coupling condition.
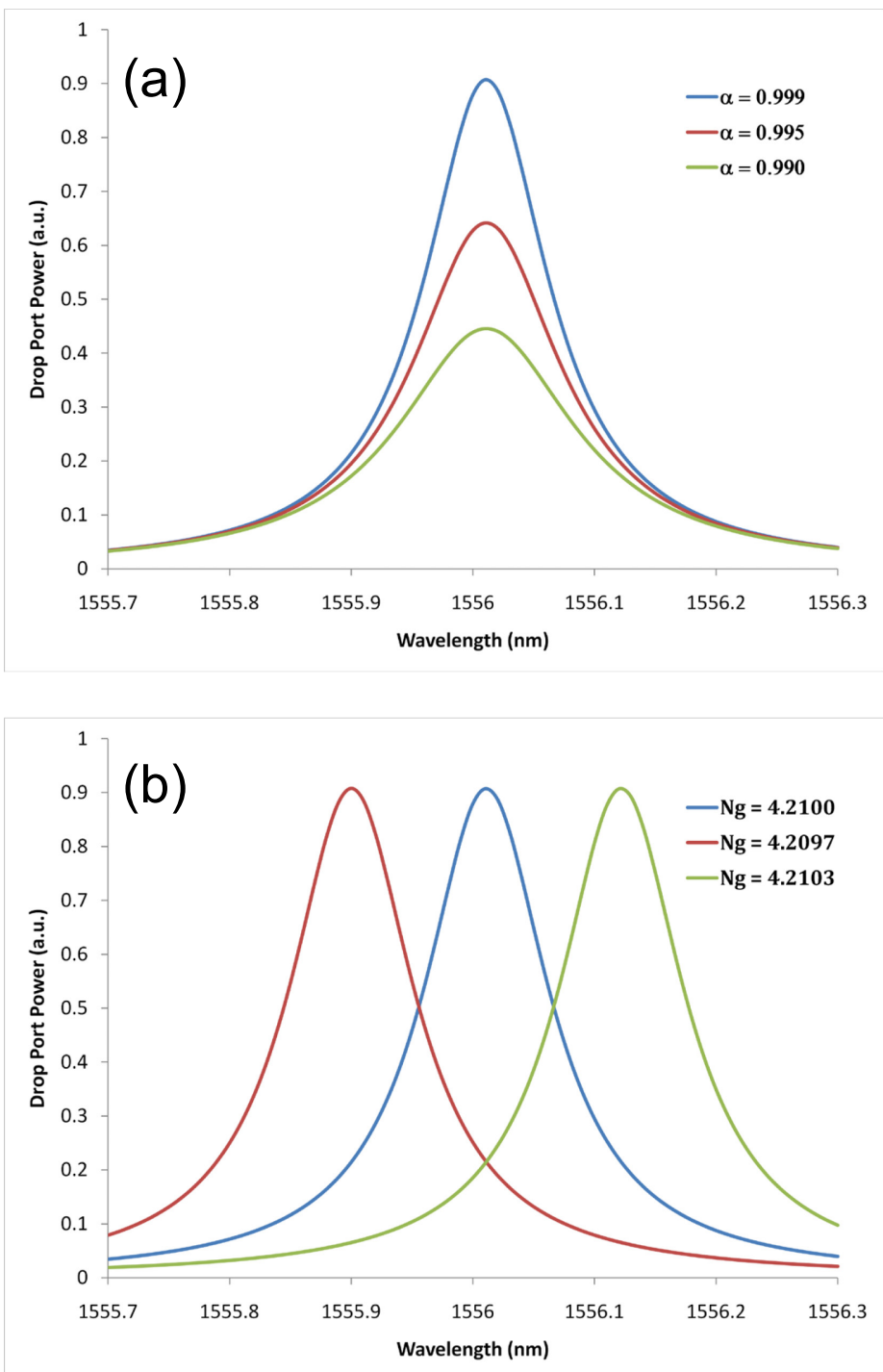


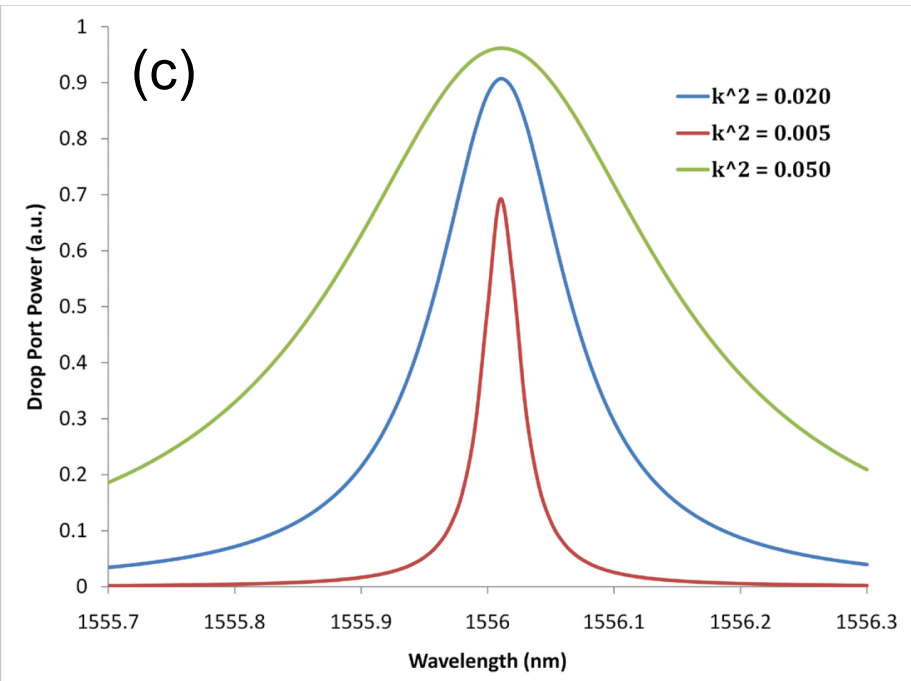

Figure 2.2 Calculated drop port transmission spectra of a microring resonator $\left(\alpha=0.999, \kappa^{2}=0.02, \mathrm{r}=\right.$ $5 \mu \mathrm{m}$ and $n_{g}=4.21$ ) with (a) tunable resonator loss, (b) tunable resonant wavelength, and (c) tunable coupling ratio.

\subsection{Thermooptic Wavelength Tuning of Microring Resonator}

The thermooptic coefficient is a measure of refractive index change of a material when a temperature perturbation occurs. To find the thermooptic wavelength coefficient for a specific mode, we need to link the effective index change and group index change of the mode with the temperature change.

Consider the waveguide of microring resonator with a propagation constant $\beta$ at resonant frequency $\omega$ at temperature $T$. The thermooptic tuning can be described as a two-step process: first, the temperature $T$ is changed by a small amount $\Delta T$, which changes the propagation constant by $\Delta \beta_{T}$ and the resonance is no longer $\omega_{0}$. Then, the frequency is changed which induces another propagation constant change of $\Delta \beta_{\omega}$. For this process, we have

$$
\Delta \beta_{T}+\Delta \beta_{\omega}=0
$$


Note that $n_{e f f} \equiv \beta / k=\beta c / \omega$ and $n_{g} \equiv \partial \beta / \partial k=\partial \beta / \partial k=(\partial \beta / \partial \omega) \cdot c$, the equation above can be expanded as

$$
\begin{gathered}
\frac{\omega}{c} \frac{\partial n_{e f f}}{\partial T} \Delta T+\frac{n_{g}}{c} \Delta \omega=0 \\
\frac{\Delta \omega}{\omega}=-\frac{1}{n_{g}} \frac{\partial n_{e f f}}{\partial T} \Delta T
\end{gathered}
$$

Where $c$ is the light speed in vacuum.

In terms of wavelength tunability, we have

$$
\frac{\Delta \lambda}{\lambda} \cong-\frac{\Delta \omega}{\omega}=\frac{1}{n_{g}} \frac{\partial n_{e f f}}{\partial T} \Delta T
$$

The thermooptic wavelength tuning coefficient is

$$
\frac{\Delta \lambda}{\Delta T}=\frac{\lambda_{0}}{n_{g}} \frac{\partial n_{e f f}}{\partial T}
$$

Now, the wavelength tuning is connected with the group index $\left(n_{g}\right)$ and the effective thermooptic coefficient of the waveguide mode $\left(\partial n_{e f f} / \partial T\right)$ which depends on the overlap intergral of the mode with the thermal index perturbation. For the waveguide with a single core material and a single cladding material, $\partial n_{e f f} / \partial T$ the coefficient can be written as

$$
\frac{\partial n_{\text {eff }}}{\partial T}=\frac{\partial n_{\text {eff }}}{\partial n_{\text {core }}} \frac{\partial n_{\text {core }}}{\partial T}+\frac{\partial n_{\text {eff }}}{\partial n_{\text {cladding }}} \frac{\partial n_{\text {cladding }}}{\partial T}
$$

From Equation (2.39), one can find that thermooptic effects of both the core material and the cladding material contribute to the wavelength tuning. Their contributions are $\partial n_{e f f} / \partial n_{\text {core }}$ and $\partial n_{e f f} / \partial n_{\text {cladding }}$, respectively. The contributions are related to the distribution of the optical field. Generally, if most of the field 
concentrated in the core, the contribution of thermooptic effect of the core material is bigger compared to that of the cladding material.

For a waveguide structure with most of optical field confined in the core, core material with a larger thermooptic coefficient yields a larger wavelength tuning with the same temperature change. Thus, materials with large thermooptic coefficients are preferred as core material of a waveguide for applications which need wide thermooptic tunable range. Also, waveguide designs which allow higher optical confinement in the core are preferred if the thermooptic coefficient of the core material is much higher compared to that of the cladding.

The thermooptic tuning effect for the core and cladding could add or compete with each other depending on the sign of the thermooptic coefficient for the materials. Typically, the thermooptic coefficient of polymers is negative, while that of the semiconductors like silicon is positive. Designs may be chosen to make athermal resonators which are insensitive to temperature change, by balancing the thermooptic tuning effect of the silicon core and polymer cladding. Such a thermal design is common in PLC waveguide design [77]. 


\section{DESIGN OF THE THERMALLY TUNABLE FILTER}

In the previous chapter, we briefly introduced the theories of microring resonator as well as its tuning mechanism. When using a microring resonator as a tunable filter, many parameters and issues need to be considered to achieve an optimal design. Here are some general considerations for the tunable filter: optical loss, FSR, filter tunable range, quality factor, tuning speed, device feature size, and fabrication tolerance.

In this chapter, the design of the thermally tunable filter was studied, taking into consideration the factors stated above. Optical and thermal simulations were performed to verify and optimize the design. Some key parameters were calculated from the simulation result, which will be used to compare with the real device fabricated.

In this work, two simulation programs called BeamProp and FullWave (both made by Rsoft Design Group) were used for modeling the optical properties of the device. The two programs are based on beam propagation method (BPM) and finite-difference time domain metrod (FDTD), respectively. The thermal simulations were carried out using the heat transfer module in Comsol Multiphysics simulation software package (formerly FEMLAB).

All simulations were done on a HP desktop equipped with Intel $2.8 \mathrm{GHz}$ Core2 Duo CPU and 4GB memory. Usually, larger memory size and longer simulation time are needed for simulations with finer simulation grid size. In these simulations, we chose the finest simulation grid size supported by the computer in order to get an accurate result. 


\subsection{Optical Design of the Tunable Filter}

\subsubsection{Waveguide dimension}

In integrated photonic devices, waveguides serve as transmission media. For ease of fabrication, we usually use strip waveguides with rectangular cross-sections. The device was designed based on a silicon waveguide embedded in silica with rectangular cross-section of $450 \mathrm{~nm} \times 250 \mathrm{~nm}$. This dimension was used widely in other silicon photonics devices $[3,52,62]$. Due to the asymmetric dimension, it has different optical properties when transmitting light with different polarizations.

In this work, electromagnetic waves with electrical fields polarized in the plane of the chip are referred as TE polarized; and with electrical fields polarized normally to the plane are referred as TM polarized. This nomination is commonly acceptable to describe the polarization states in integrated photonics. In general, dielectric waveguides with high index contrast support modes with hybrid polarization. However, the waveguides usually support modes of interest, that are quasi-TE (TE-like) or quasi-TM (TM-like). And in this work, the nomenclature is simplified to TE and TM to refer to these modes. Optimization of a waveguide in this chapter was done with TE polarization only, however, the performance for both polarizations is considered.

The waveguide with cross-section of $450 \mathrm{~nm} \times 250 \mathrm{~nm}$ is single mode for both TE and TM polarized light at the proposed working wavelength of $\sim 1.55 \mu \mathrm{m}$. The crosssection is close to the maximal dimension for single-mode SOI for TE mode with which light is maximally confined in the core. With higher light confinement in the core, waveguides can be sharply bent without significant loss. And given much higher 
thermooptic coefficient of $\mathrm{Si}\left(1.84 \times 10^{-4} /{ }^{\circ} \mathrm{C}\right)$ [35] compared to $\mathrm{SiO}_{2}\left(\sim 10^{-5} /{ }^{\circ} \mathrm{C}\right)$, higher light confinement in the core provides higher wavelength tuning with same the temperature change. The TE and TM mode fields were simulated at wavelength of $1.55 \mu \mathrm{m}$ by beam propagation method (BPM) using Beamprop software as shown in Figure 3.1 (a) and (b), respectively. The dark line shows the boundary of the silicon core. The refractive indexes for $\mathrm{Si}$ and $\mathrm{SiO}_{2}$ were assumed to be 3.48 and 1.46 in the simulation at the working wavelength around $1550 \mathrm{~nm}$. The same indexes are assumed in other works $[60,61]$ using similar SOI substrate. The effective refractive indexes were calculated by the simulation software to be 2.51 for TE mode and 2.05 for TM mode. In our design, the thickness of upper cladding $\mathrm{SiO}_{2}$ was set to be $1.5 \mu \mathrm{m}$.
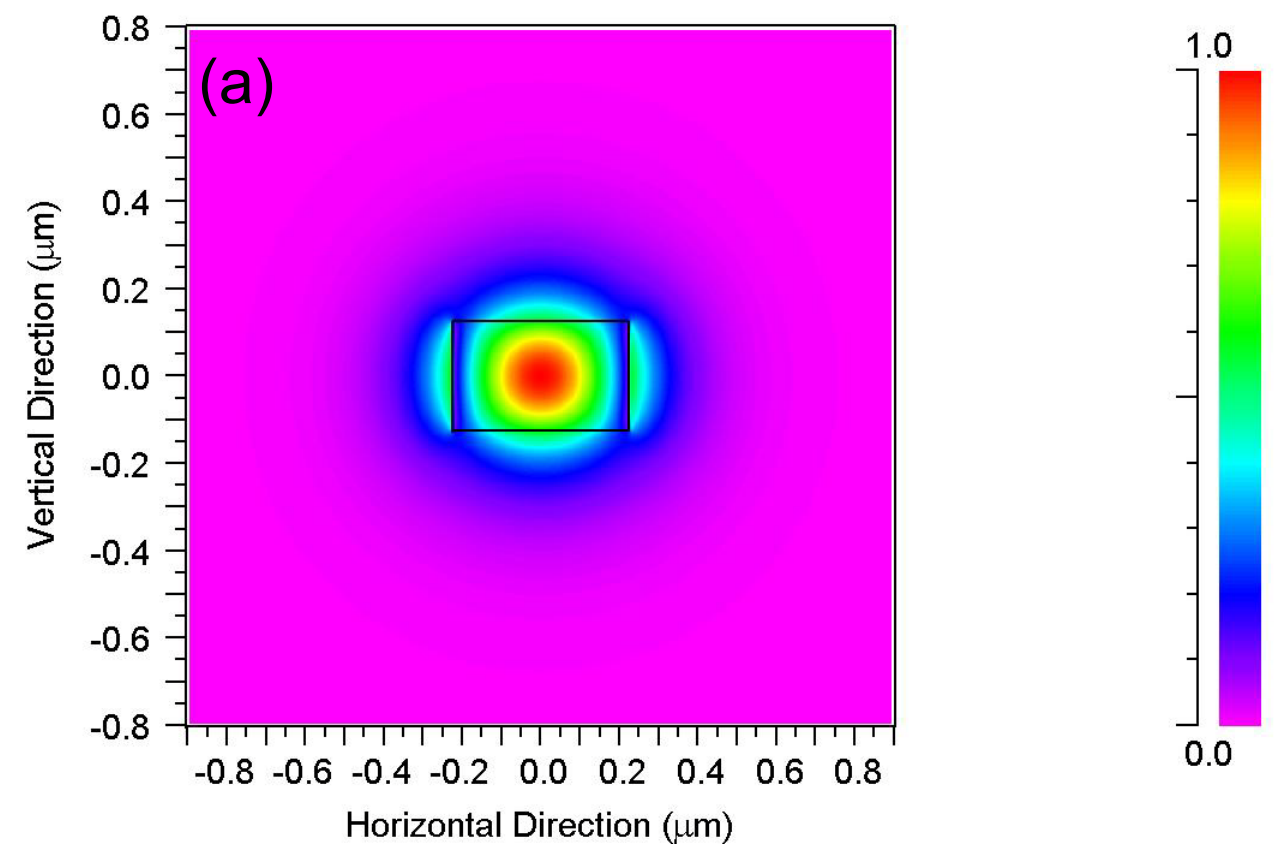

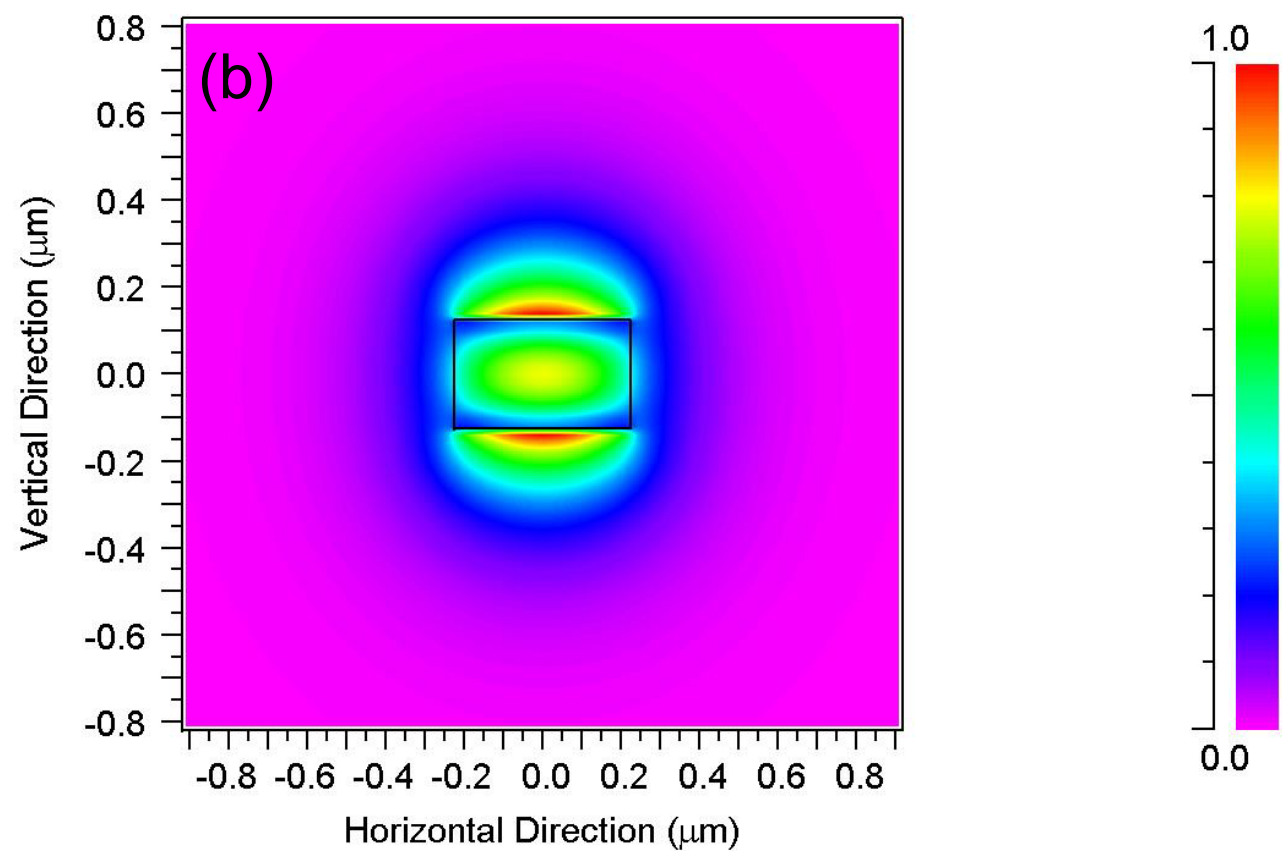

Figure 3.1 Simulated field distributions for fundamental (a) TE and (b) TM modes at 1550nm wavelength. Dark lines shows the boundary of the silicon core.

\subsubsection{Microring radius}

The FSR of a microring resonator is inversely proportional to the ring radius according to Equation (2.20) in the previous chapter. A smaller ring radius is needed to achieve higher FSR. However, as the radius gets smaller and smaller, there will be significant bending loss which will limit the quality factor (Q). In our design, a $5 \mu \mathrm{m}$ radius microring was used where the bending loss can be negligible in similar devices [42], however, an acceptable quality factor has been achieved in smaller microrings [43, 4]. 


\subsubsection{Microring- waveguide gap}

A high Q is usually desired for a resonator. For our microring resonator, smaller filter linewidth will be achieved with higher Q, which means more non-overlapping channels can be located within the tunable range. However, when the Q is too high, it limits the maximum modulation frequency of a channel. For a modulator working at 10$20 \mathrm{Gbit} / \mathrm{s}$ [3], a moderately high operating Q on the order of 10,000 would be appropriate. The Q corresponds to an optical bandwidth of $\sim 20 \mathrm{GHz}$. As the intrinsic loss and bending loss of the microring is negligible, the operating $\mathrm{Q}$ was determined by the power coupling efficiency $\kappa^{2}$ according to Equation (2.28) and (2.30). The straightforward way of tuning the coupling efficiency is to adjust the gap width between the straight waveguide and the microring.

(a)

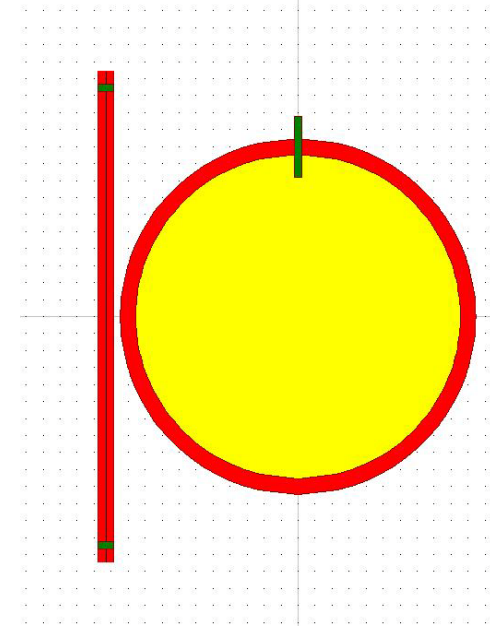

(b)

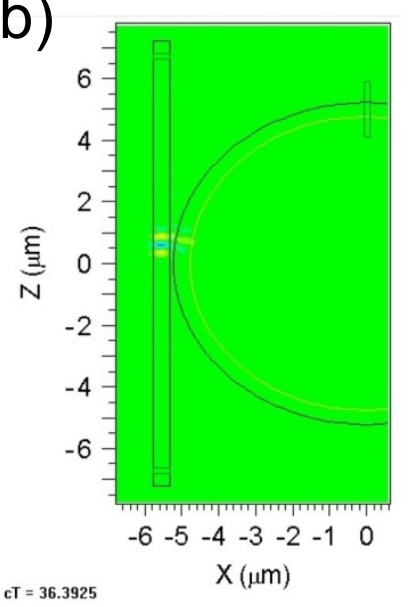

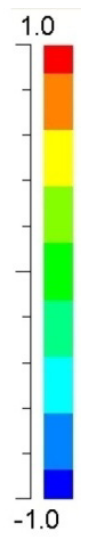

Figure 3.2 (a) Schematic of the simulated structure with a straight waveguide coupled with a $5 \mu \mathrm{m}$-radius microring. The green boxes are the power monitors. (b) Simulated electrical field distribution on crosssection of the device demonstrating the coupling between straight waveguide and microring. Color indicates the amplitude of electrical field $\mathrm{E}_{\mathrm{x}}$.

To determine the gap width, a Finite-difference time-domain (FDTD) simulation using Fullwave software from Rsoft was performed with different gaps between a straight 
waveguide and a $5 \mu \mathrm{m}$ radius microring. The schematic is shown in Figure 3.2(a). A $450 \mathrm{~nm} \times 250 \mathrm{~nm}$ cross-section was assumed in the simulation where the refractive index was set to 3.48 in the channel and 1.46 outside of the channel. A pulse was input from the lower end of the straight waveguide and measured by the monitors located at different locations shown as green boxes in the plot. The Fourier transform of the detected time response was done to find out the coupling efficiency for different wavelengths. Because of the limit of the computer memory, the simulation grid was set to $0.05 \mu \mathrm{m}$; however, it is accurate enough for us to determine the gap width. Figure 3.2(b) demonstrates the coupling between the waveguide and the ring. The power coupling efficiency at $1.55 \mu \mathrm{m}$ for different gaps is plotted in Figure 3.3 with the corresponding Q shown on the right. From Figure 3.3, one can find that the minimal gap to obtain a Q of 10,000 is $180 \mathrm{~nm}$. The simulation does not include propagation losses along the microring such as losses caused by the sidewall roughness. The losses will decrease the Q. In the design, we choose a slightly larger gap of $200 \mathrm{~nm}$ with simulated Q of 14,800. The higher theoretical $\mathrm{Q}$ in the design gives some margin for possible $\mathrm{Q}$ degradation caused by the losses. The 200nm gap size can be well controlled with electron-beam lithography, which makes the design practical at the fabrication stage. 


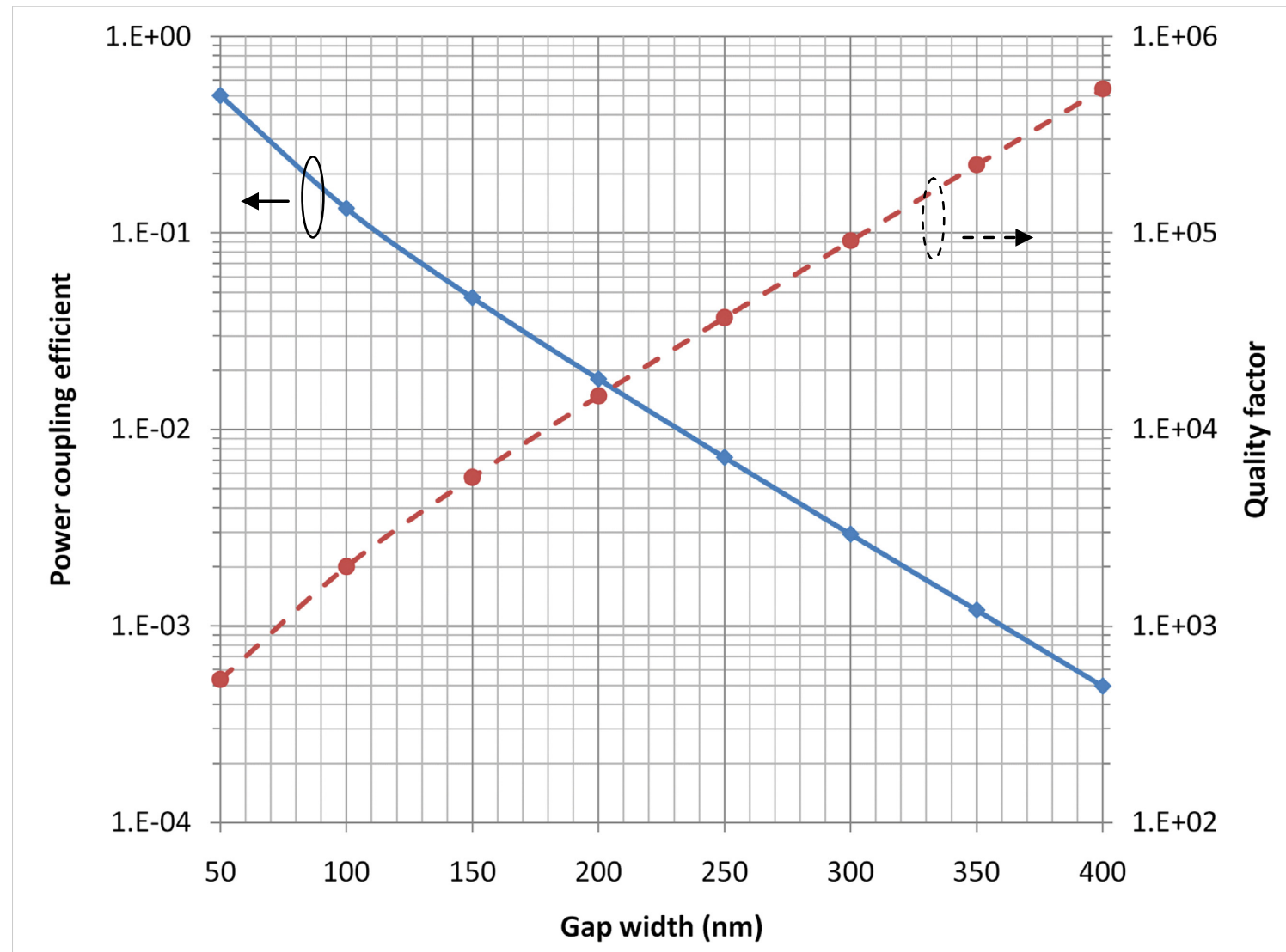

Figure 3.3 Power coupling coefficient and corresponding Q for different gap width.

The simulation was also performed for TM mode with gap of $200 \mathrm{~nm}$. The power coupling efficiency of both TE and TM mode with wavelength range of 1500-1650nm was plotted in Figure 3.4(a) and (b). For the same wavelength, the coupling efficiency for TM mode is much higher than the one for TE mode, indicating a much lower Q for TM mode. One can see that the coupling efficiency increases with wavelength for both modes. This is because the tails of the modes extend more outside of the core with larger wavelength and increase the overlap between the mode of the straight waveguide and microring waveguide. 

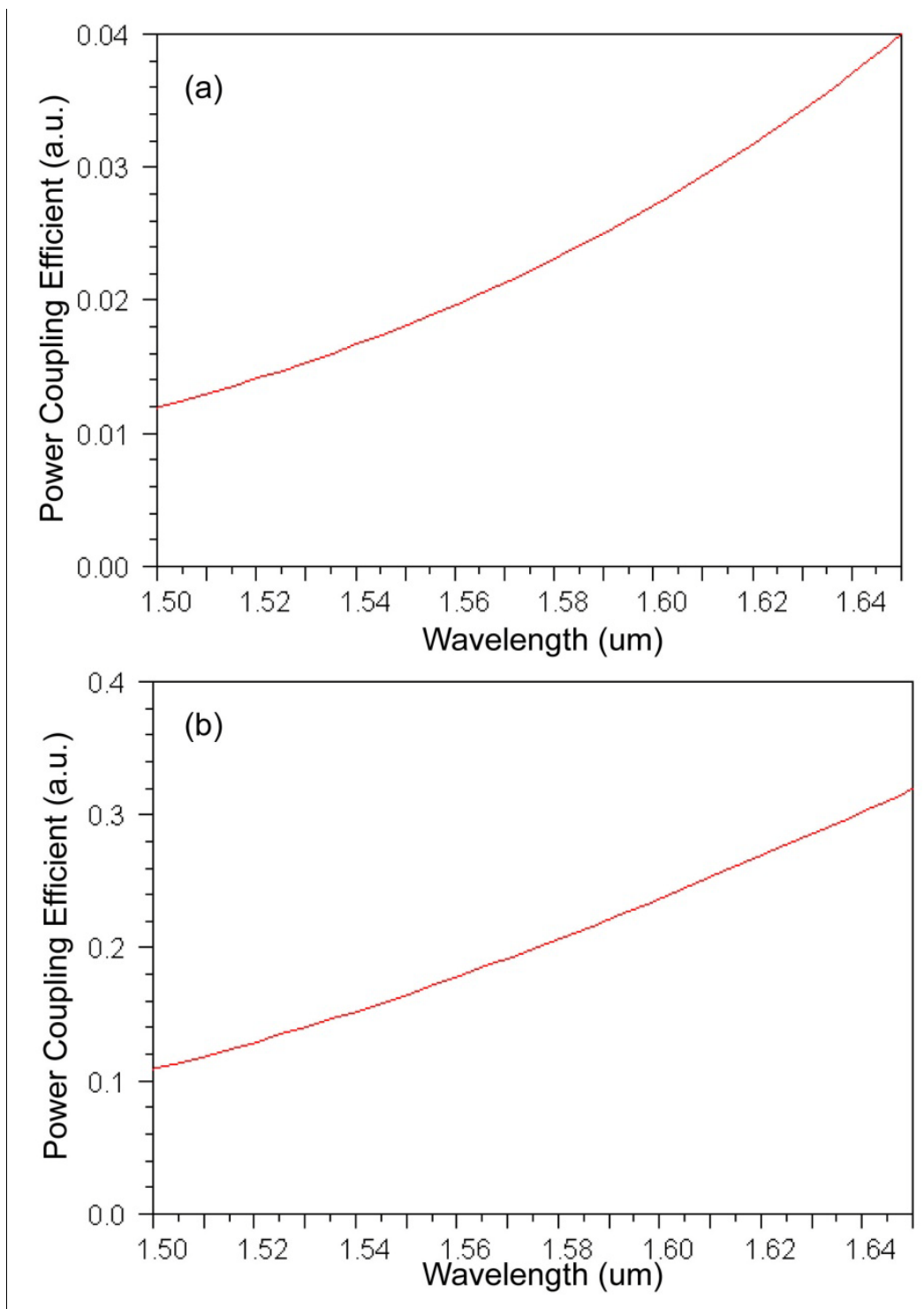

Figure3.4 Power coupling coefficient of (a)TE mode and (b) TM mode between straight waveguide and a $5 \mu \mathrm{m}$-radius microring with gap of $200 \mathrm{~nm}$.

\subsubsection{Cladding thickness}

The absorption loss by the metal heater above the microring could impair Q by a large degree if it is close to the optical field propagating in the microring waveguide. To avoid strong absorption, the thickness of the top cladding layer needs to be big enough. From the simulated mode field distributions for TE and TM modes as shown in 
Figure 3.1, one can see that the field amplitudes at $0.8 \mu \mathrm{m}$ above the center plane of the waveguide ( $0.925 \mathrm{um}$ from the bottom of the waveguide) are very small. To leave some margin for thickness error during cladding dioxide deposition, we designed the heater $1.5 \mu \mathrm{m}$ above the waveguide. The absorption loss simulation result can be found elsewhere [62], which indicates a negligible loss on the order of $\sim 1 \times 10^{-4} \mathrm{~cm}^{-1}$ with upper cladding of $1.25 \mathrm{um}$.

\subsubsection{Overall optical design}

The optical design of the filter is shown in Figure 3.5. The outline shows the size of the chip we designed. The size of the chip is about $6 \mathrm{~mm}$ in length and $4 \mathrm{~mm}$ in width. The size approaches the minimal size capable of being manipulated comfortably with tweezers. The input and output waveguides were offset, and both ends are with nanotapers [44], which make characterization convenient. The nanotaperes can theoretically reduce insertion loss to below $1 \mathrm{~dB}$ and was proved by experiment to greatly increase the fiber to waveguide coupling efficiency. The input port and drop port were

designed perpendicularly to each other instead of parallel. Compared to the standard configuration in the simulation, this configuration is expected to have identical optical response, while being more convenient to locate multiple filters along the common through port for the applications which will be described later. The two waveguides were separated by $0.5 \mu \mathrm{m}$ at the intersection to avoid crosstalk between them. 


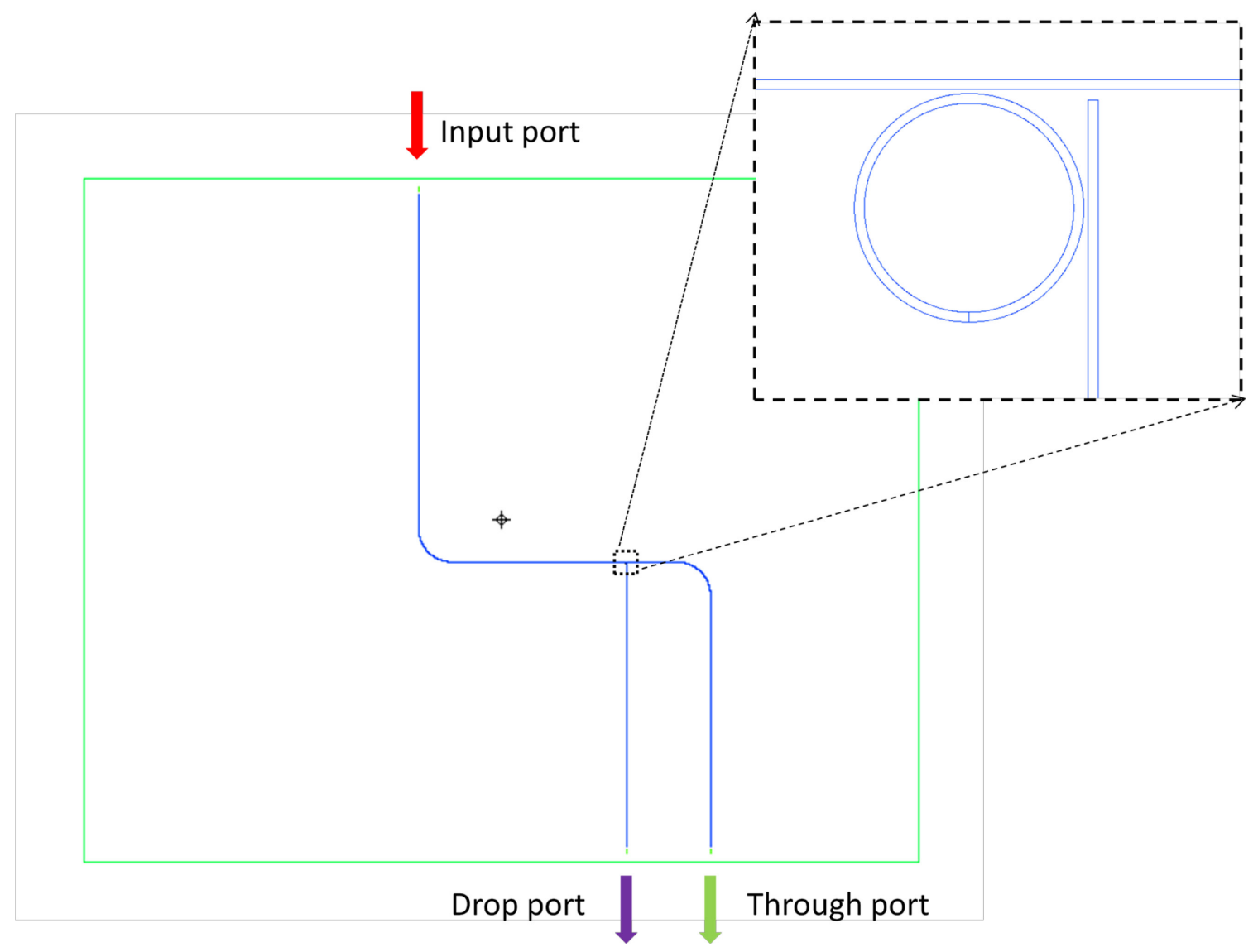

Figure 3.5 Optical design of the tunable filter chip. Inset: magnified picture of the design of the microring resonator

\subsubsection{Verification of optical design}

To verify the optical design, a FDTD simulation was performed with the structure

plotted in Figure 3.6. A pulse was sent from the lower end of the left straight waveguide. Time monitors were set at the through port and drop port. Similar to the coupling simulation, the Fourier transform was done with the time response of the power at the through port and the drop port to obtain the spectra. Simulations were performed for both TE and TM modes and the spectra in range of 1520 to $1620 \mathrm{~nm}$ are shown in Figure 3.7(a) and (b). The simulations consume a lot of time and memory. So the space grids and light 
propagation time were limited to the capability of the computer. With longer propagation time and finer space grid, the simulation results get more accurate. In the simulation the space grids are set to be $0.05 \mu \mathrm{m}$ and the FDTD stop time was set to $2^{19} \times$ time_step in the simulation. A FSR of $\sim 18 \mathrm{~nm}$ was observed from both TE and TM spectra in this wavelength range. A much larger resonance linewidth was found for TM mode resonances, indicating a lower Q compared to TE mode. The linewidth may not reflect the real Q for the system because of the limited simulation time for the Fourier transform. But it reflects its nature and trends.

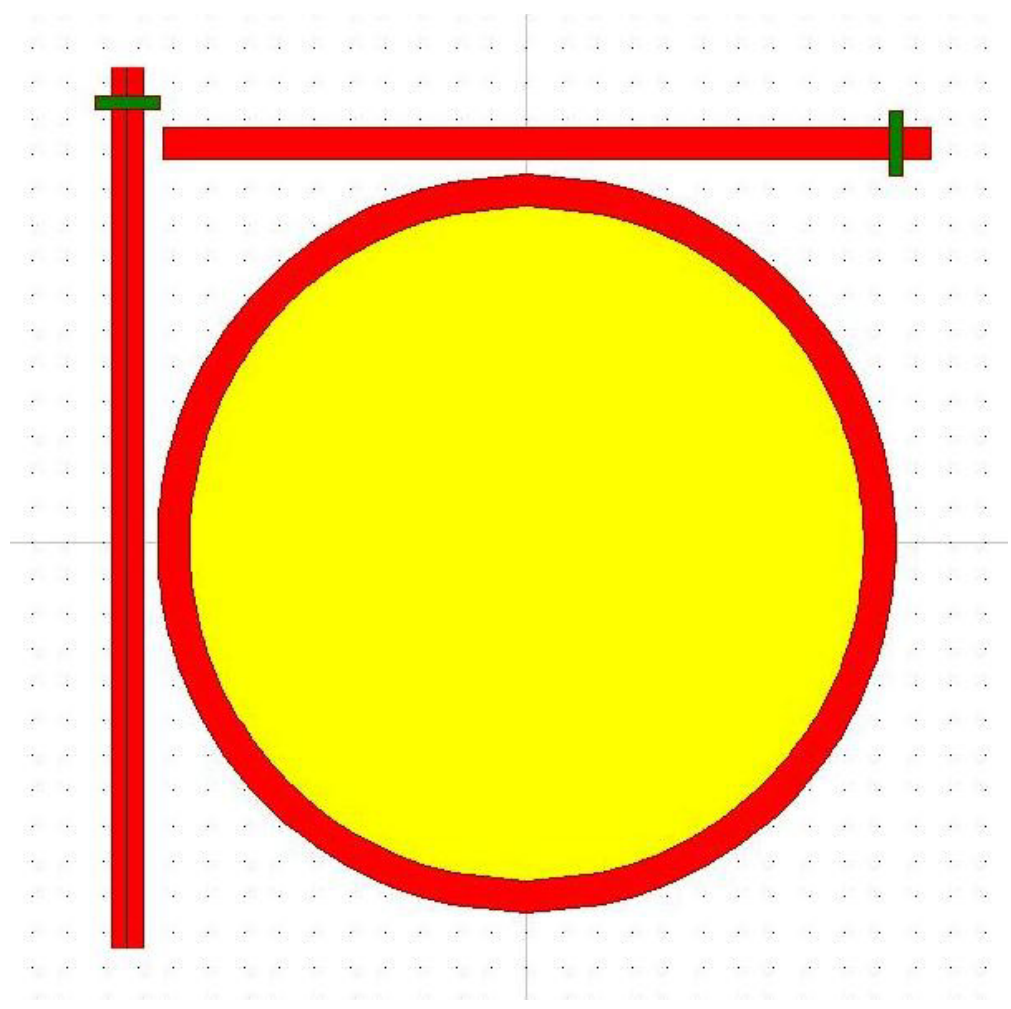

Figure 3.6 Schematic of simulated structure in FDTD simulation of the microring with input and output waveguides in perpendicular 
(a)
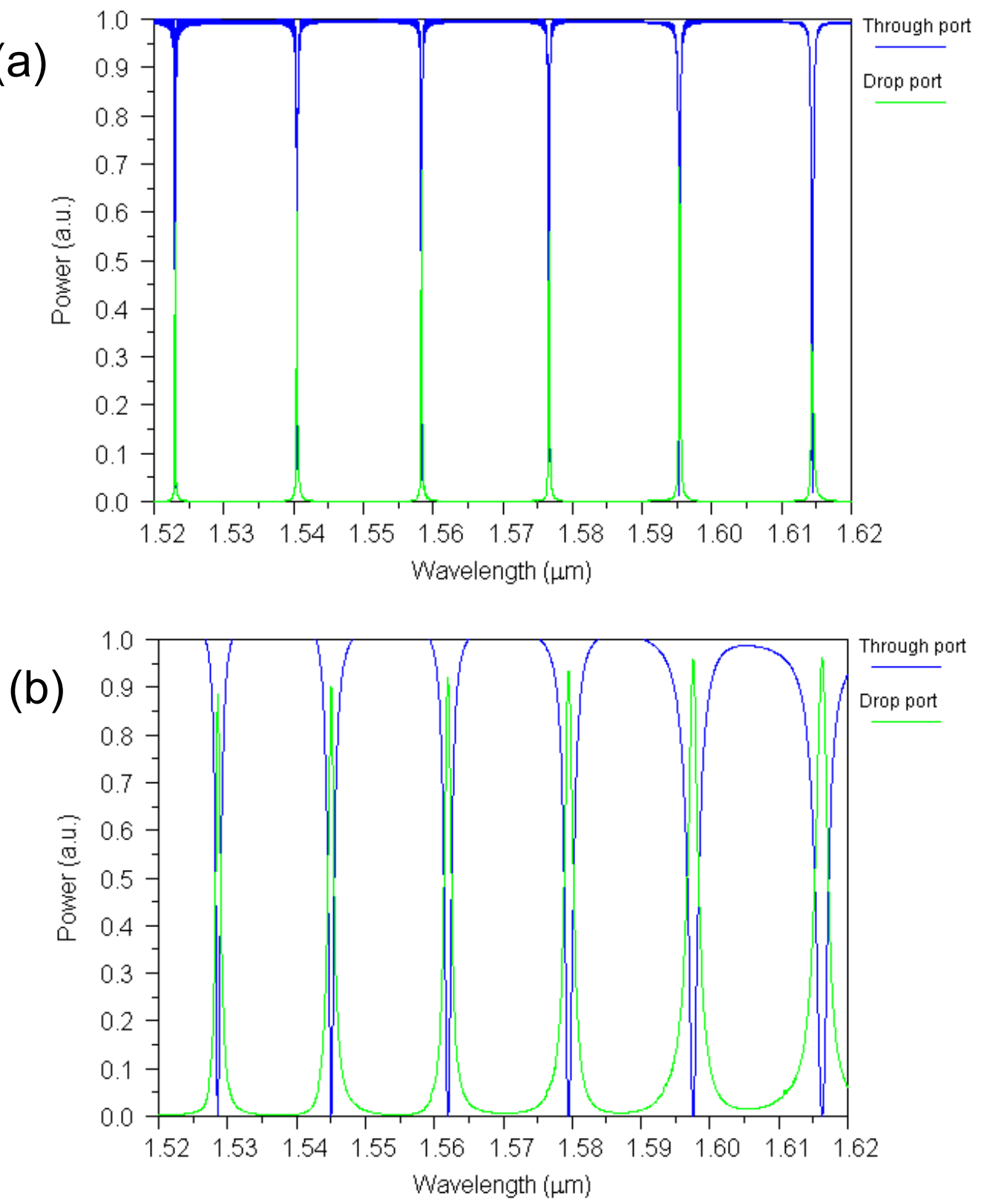

Figure 3.7 Simulated through port and drop port spectra of (a) TE mode (b) TM mode

Another FDTD simulation was carried out with the TE mode input with continuous wave at the measured resonance wavelength of $1.5584 \mu \mathrm{m}$ to verify the spectrum response. The electrical field $\mathrm{E}_{\mathrm{x}}$ distribution at the end of the simulation time of $2^{19} \times$ time_step and the time response of the through port and drop port power are shown 
in Figure 3.8(a) and (b). The simulation helps to understand the power accumulating process in the microring due to constructive interference between the coupled light and the circulating light. Eventually, the drop port and through power will get stabilized. Note that the drop port power in this simulation will not reach unity because it is not in a critical coupling condition although on resonance.
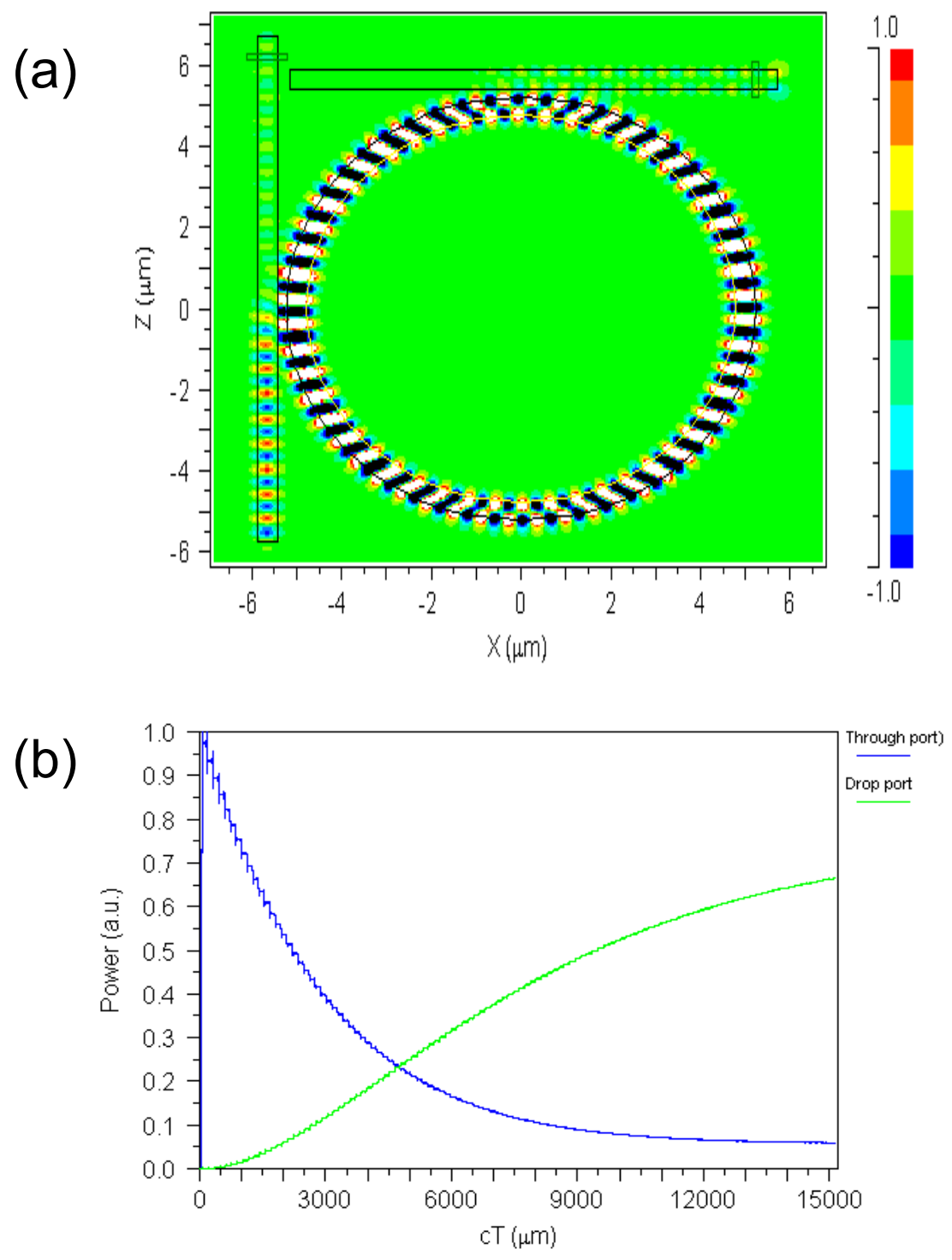

Figure 3.8 (a) Simulated electrical field $\mathrm{E}_{\mathrm{x}}$ distribution of the device at the center plane of the waveguide and the ring (b) Simulated time response of the through port power and the drop port power. The time scale shows the free space propagation length in $\mu \mathrm{m}$. The simulation was done with continuous wave input at a resonance wavelength of $1.5584 \mu \mathrm{m}$ 


\subsection{Thermal Design of the Wavelength-tunable Filter}

\subsubsection{S-shape micro-heater design and verification}

A resistive micro-heater was proposed to provide heat to thermally tune the resonance of the microring resonator. The fabrication capability and cost was taken into consideration when designing the heater. Photolithography using a contact aligner was proposed to generate the heater pattern to avoid the high cost of ebeam lithography and stepper. However, the relative low resolution $(1-2 \mu \mathrm{m})$ of the contact photolithography compared to the feature size of the microring waveguide $(0.45 \mu \mathrm{m})$ greatly limits the power efficiency of the heater. To avoid the heater filament breaking due to fabrication defects, the filament width of our micro-heater was set to be $5 \mu \mathrm{m}$. To demonstrate significant wavelength tuning with most of the power generated at the heater area, we choose Nichrome, an alloy of nickel (80\%) and chromium $(20 \%)$ with high resistivity of $1.5 \times 10^{-6} \Omega \mathrm{m}$, as the heater material. A thickness of $100 \mathrm{~nm}$ was proposed for the heater which can be controlled very well when fabricated with thermal evaporation. An S-shape heater with a linewidth of $5 \mu \mathrm{m}$ was designed as shown in Figure 3.9 to provide uniform heating in an area of approximately $50 \mu \mathrm{m} \times 50 \mu \mathrm{m}$, allowing misalignment between heater and the microring. Gold electrical feedlines with linewidth of $10 \mu \mathrm{m}$ were designed as well as sub-millimeter-size electrode pads for probing and wire bonding located at the side of the chip. The center of the heater was vertically aligned with the center of the microring resonator on the optical layer of the device. 


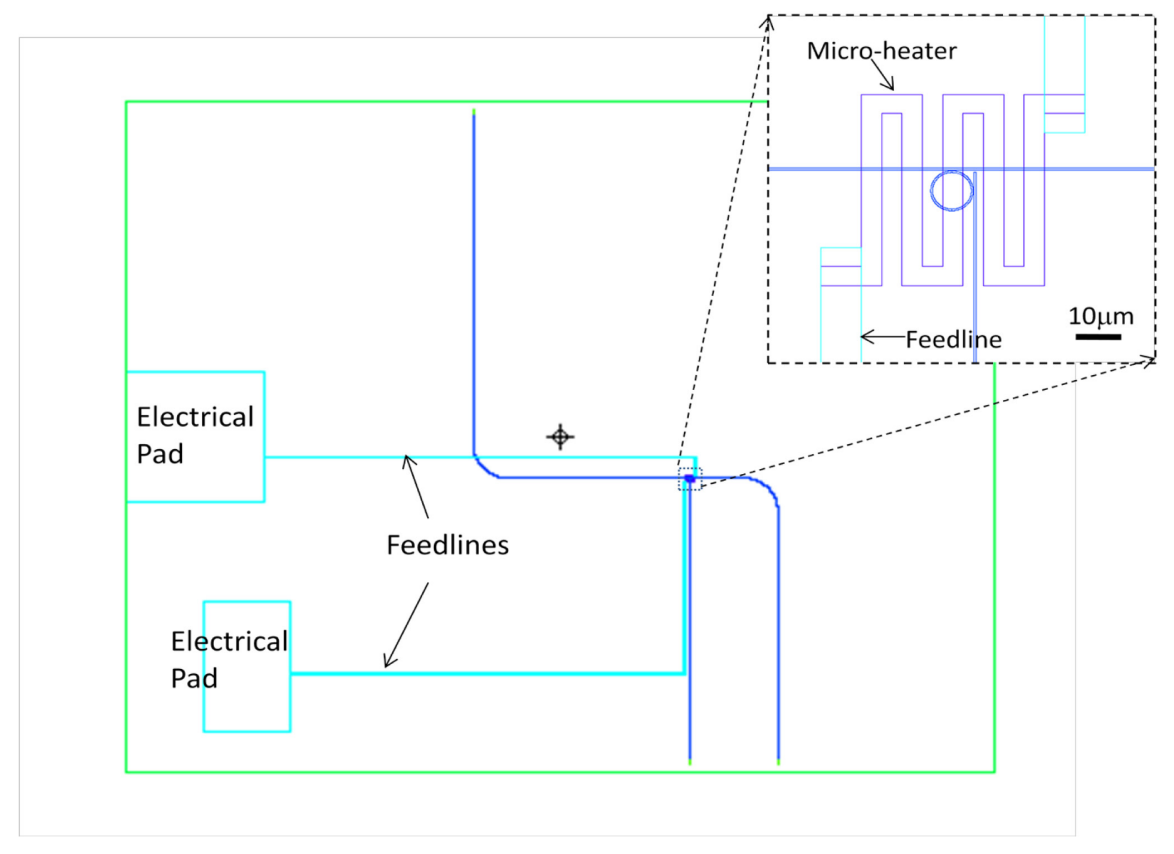

Figure 3.9 Design of micro-heater, feedlines and electrode pads. Inset: magnified picture of the designed micro-heater aligned with the microring

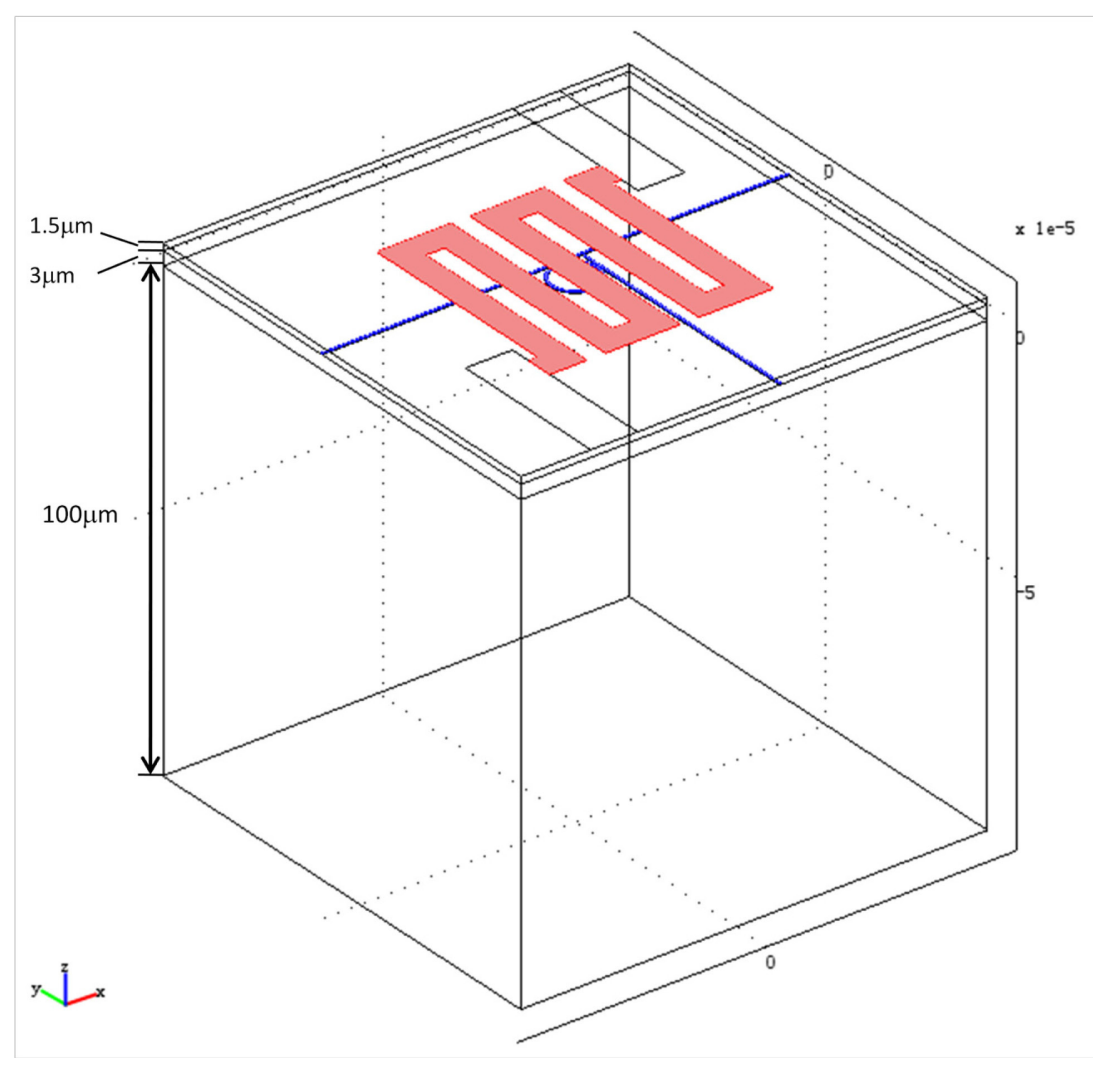

Figure 3.10 Design for the thermal simulation using Comsol Multiphysics 
To estimate the heater performance and to help further optimization, a 3D thermal simulation of the device was carried out using Comsol Multiphysics simulation package, a finite-element analysis software. The 3D simulation design was shown in Figure 3.10 with heater shown in red and the microring and waveguides shown in blue. The key simulation conditions and assumptions are summarized as below:

1. An area of $100 \mu \mathrm{m} \times 100 \mu \mathrm{m}$ and the substrate thickness of $100 \mu \mathrm{m}$ wereconsidered for the simulation although the real thickness of the substrate is $500 \mu \mathrm{m}$. The $3 \mathrm{D}$ simulation dimension is limited by the memory size of the computer. However, the result should be accurate with the reduced substrate thickness considering the $\sim 100$ time larger thermal conductivity of silicon substrate compared to the silicon oxide top layers.

2. Thermal isolation was assumed at the four side walls of the simulation cube because temperature change at those areas is negligible when heating.

3. Perfect heat sink was assumed at the back of the substrate, meaning that the temperature at the bottom was fixed at the room temperature (295K). The device initial temperature was also set to room temperature.

4. The heater and its feedlines are assumed to be thin and highly conductive layers. Thickness of $100 \mathrm{~nm}$ is assigned to the heater and feedlines.

5. The resistivity change of the heater and feedline material with temperature was neglected in this simulation.

6. Joule heating was calculated using shell, conductive media DC model in Comsol with fixed voltage set at the edges of the feedlines. And heat flux due to the DC generated heat was set to the boundaries at the surface of the heater. 
7. Natural air convection was assumed between air and the top surface of the device. The heat transfer coefficient is $\mathrm{h}=5 \mathrm{~W} / \mathrm{m}^{2} \mathrm{~K}[45]$.

First, the steady state simulation was performed with input voltage of $5 \mathrm{~V}$. A total joule power of $40.33 \mathrm{~mW}$ was obtained from the DC model, which corresponds to a total resistance of $620 \Omega$. Figure 3.11 shows the simulated temperature distributions. A significant temperature gradient was observed along the microring. There is an approximately $8.6^{\circ} \mathrm{C}$ temperature difference between the maximum and minimum temperatures on the ring. The temperature gradient is mainly due to the partial heater filament coverage of the microring. Because of the temperature gradient, the resonance shift is then proportional to the average temperature change. The average temperature change of $49.39^{\circ} \mathrm{C}$ was then estimated by integration of the temperature along the center of the microring waveguide. The average temperature change was found proportional to the heating power by repeating the simulation with different input voltage. Thus, the heating coefficient for this heater design was calculated to be $0.81 \mathrm{~mW} /{ }^{\circ} \mathrm{C}$. 


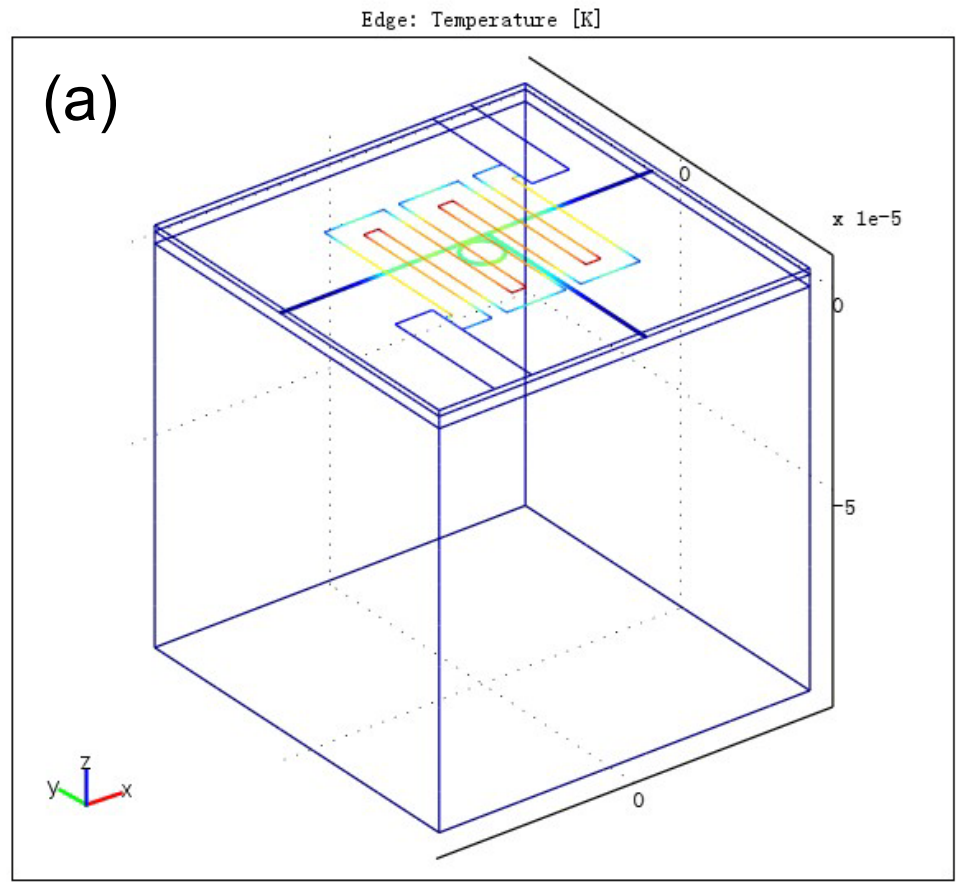

Max: 395.915
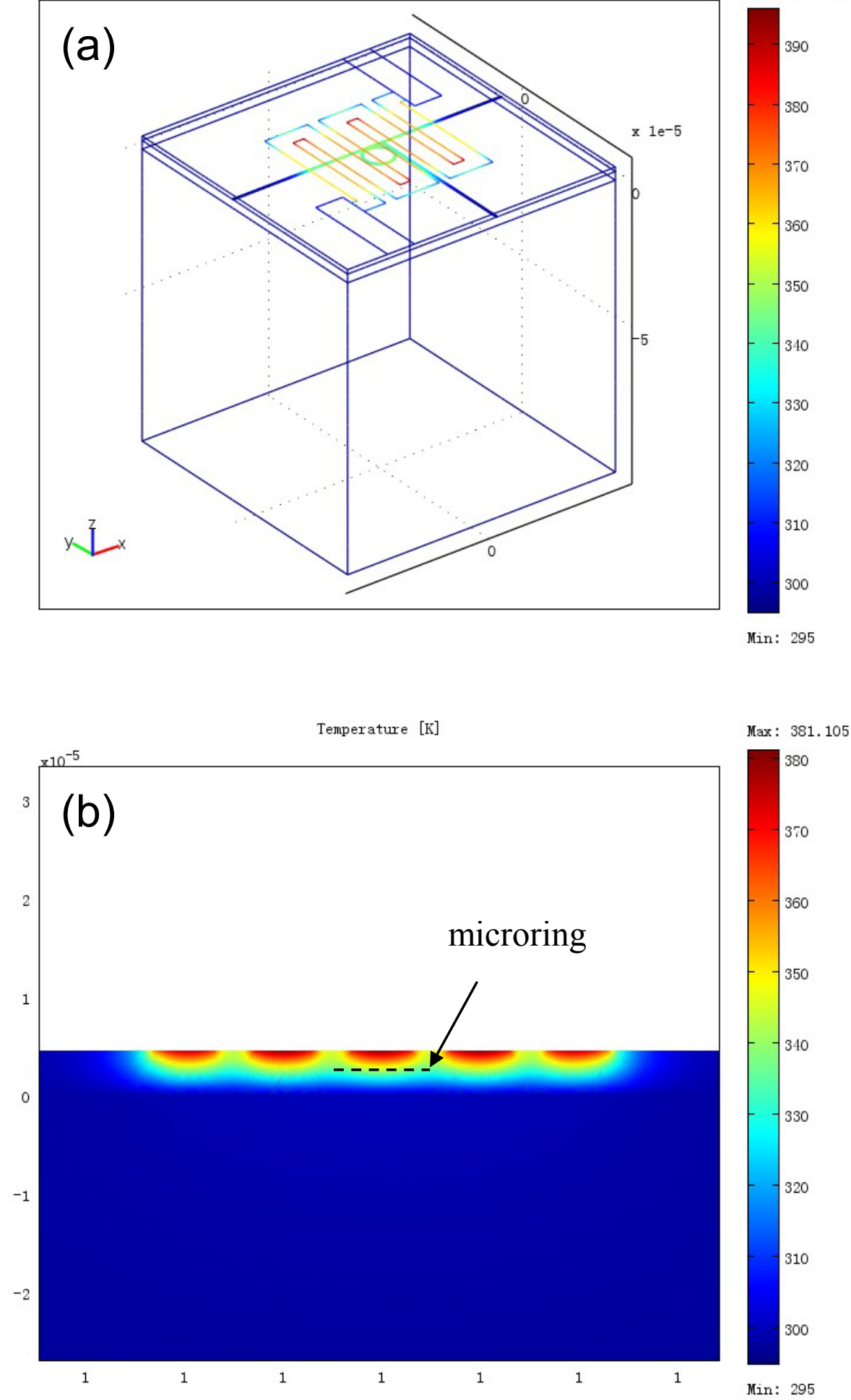


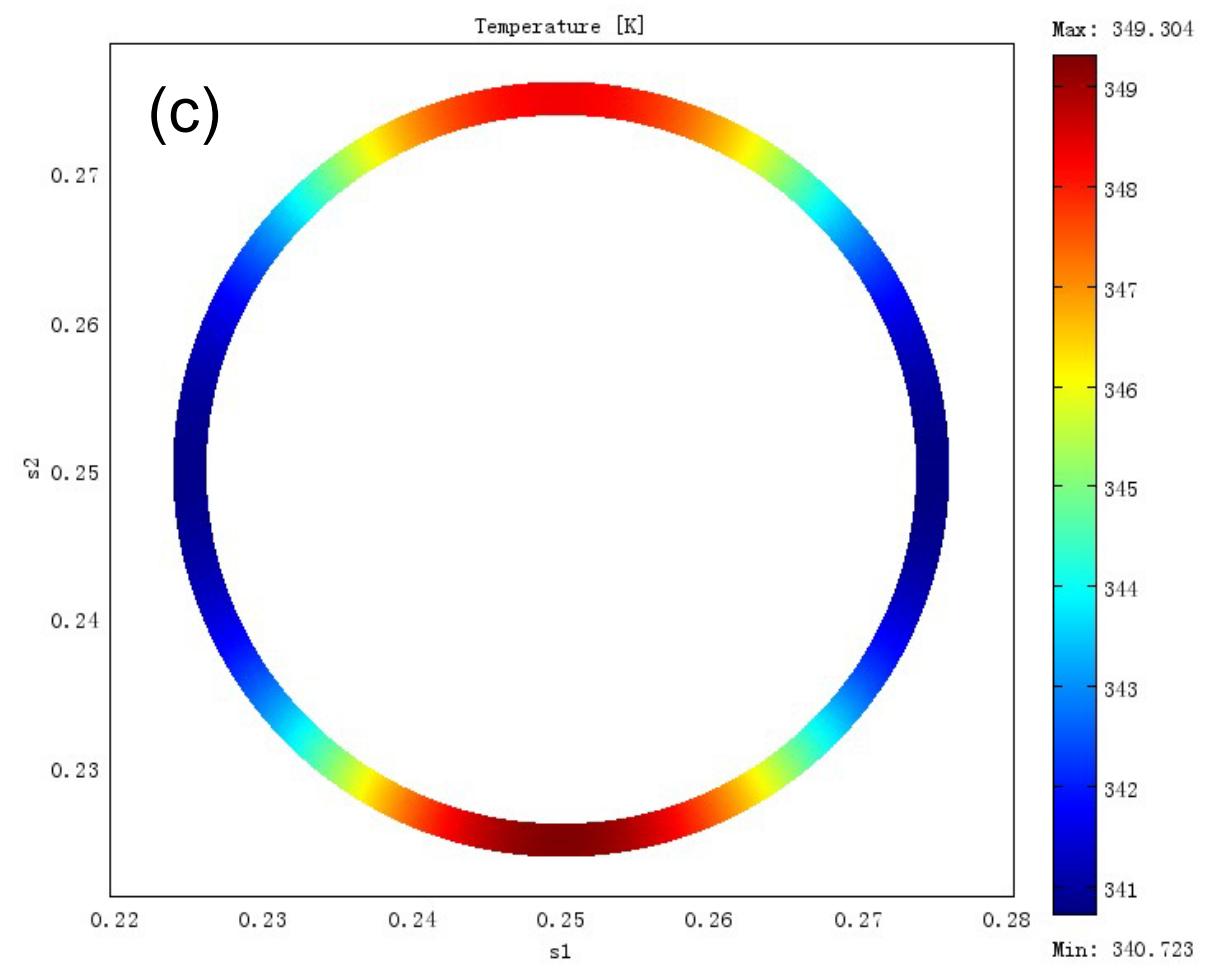

Figure 3.11 Simulated temperature distributions of (a) the whole device (b) x-z plane cross-section at the geometry center of the microring (c) along the microring . All temperatures in Kelvin. The coordinate is shown in (a)

To simulate the time response of the device, a transient simulation was done with time from 0 to $1 \mathrm{~ms}$ with a time step of $1 \mu \mathrm{s}$. The heat was taken off from $\mathrm{t}=0.5 \mathrm{~ms}$ to show the time response at cooling. The time is long enough to allow the whole system reaching thermal equilibrium at both heating cycle and cooling cycle. Figure 3.12(a) shows the simulated temperature change time response at the spots with maximum and minimum temperature change on the ring, along with the average temperature change. Besides the absolute temperature change, we also observed the difference of the time response at different parts of the microring. Figure 3.12(b) shows the time response of the normalized temperature change for first $50 \mu$ s of the heating cycle. The normalized curve shows slightly slower initial temperature change at the lower temperature part of the 
microring. The time response of the average temperature change falls in between the two normalized curves. For the cooling cycle, a similar effect is observed. The differences in time response are due to the asymmetry of the designed micro-heater. For different part of the microring, the contribution to the temperature change of the heat source right above the ring is different. And the closer to the heater source, the faster the time response.
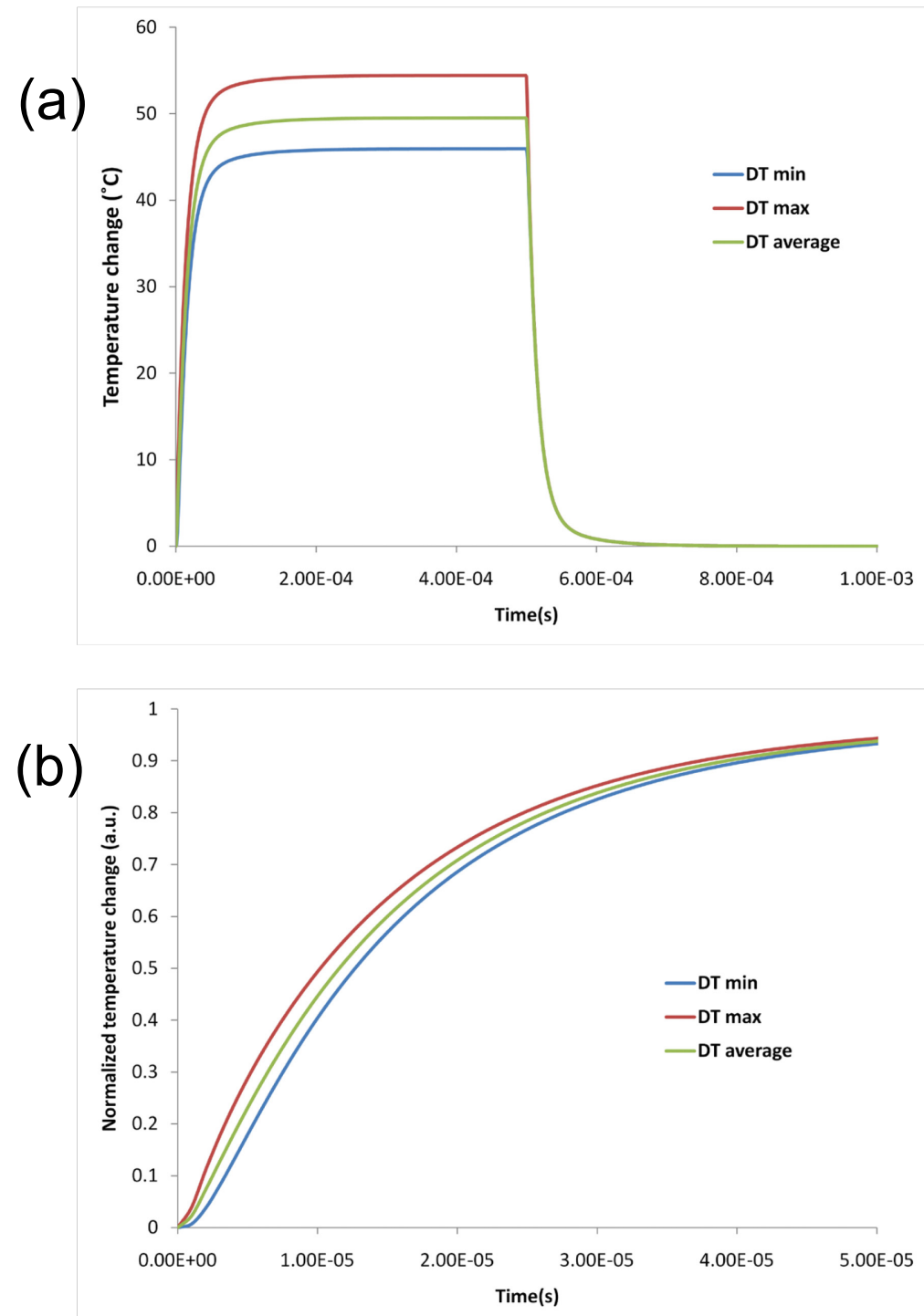

Figure 3.12 (a) Simulated time response of the temperature change of the microring; (b) Normalized simulated time response of the temperature change during the first $50 \mu \mathrm{s}$ of the heating cycle. 
The cross talk due to the heating between two adjacent tunable filters is also a key issue to be considered in the design. To enable fiber array coupling to a chip with multiple filter device, the distance between two adjacent filters was set to $250 \mu \mathrm{m}$, the diameter of a common optical fiber. Here we simulate the temperature change due to a heater with center laterally placed $250 \mu \mathrm{m}$ away from the center of the microring. Since the 3D simulation of such a big dimension is beyond the capability of our computer, we simplify the problem with a 3D cylindrical simulation where axial symmetry was assumed around the center of the microring. The generated heat was assumed to be evenly distributed over an area of a $2500 \mu \mathrm{m}^{2}$ circle (the covered area of the $50 \mu \mathrm{m} \times 50 \mu \mathrm{m}$ S-shape micro-heater) with center right on top of the center of the microring. An input power of $48.6 \mathrm{~mW}$ was assumed which corresponds to $60^{\circ} \mathrm{C}$ change of average ring temperature. The temperature was chosen according to the maximum designed tuning range. The temperature distribution on the axial cross-section is shown in Figure 3.13(a). Substrate thickness of $500 \mu \mathrm{m}$ was assumed in this simulation. Figure 3.13(b) plots the temperature distribution along the ring plane with $\mathrm{x}$-axis showing the distance to the microring center. From the plot, one can see that the temperature drops quickly outside of the heater covered area. The temperature change measured at a distance of $250 \mu \mathrm{m}$ away from the ring center is $\sim 0.2^{\circ} \mathrm{C}$, resulting in a tuning of $0.015 \mathrm{~nm}$ for the TE mode according to the optical simulation, this is much smaller compared to the linewidth of the resonance. Thus the cross talk between two adjacent filters is minimal. 

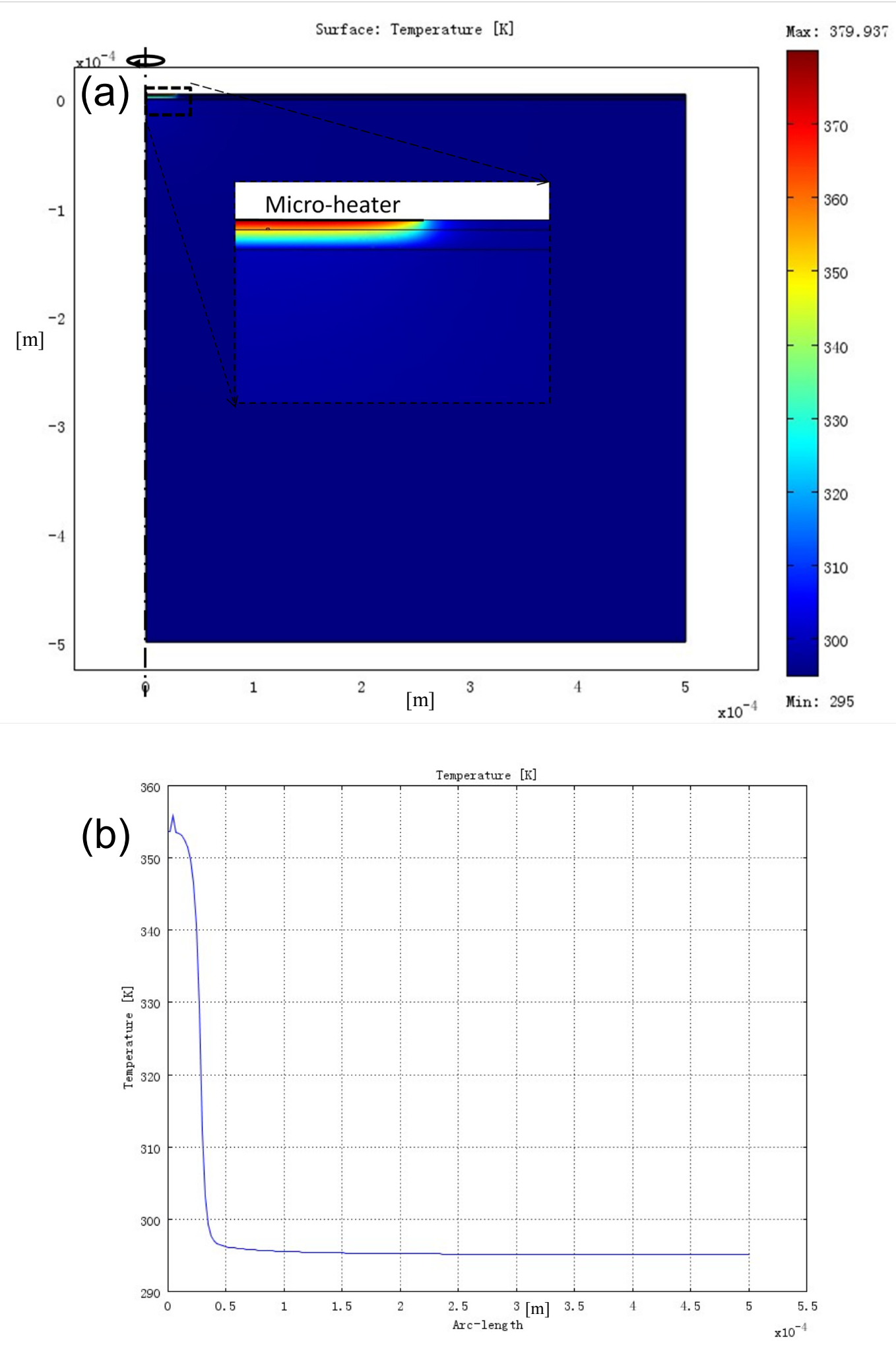

Figure 3.13 Simulated temperature distribution (a) on the cross-section (b) along x-axis across the microring 


\subsubsection{Ring-shape micro-heater design and verification}

From the simulations shown in previous section, we can see that the S-shape heater is far from ideal for this tuning application. Firstly, only a very small portion of the heating power generated contributes to the temperature change of the microring. Secondly, the large and asymmetric dimension of the heater compared to the ring hinders the time response of the filter. The design was limited by the fabrication method as stated previously. Assuming a high-resolution lithography tool (e.g., ebeam lithography) could be used for the micro-heater patterning, an optimized design was proposed as shown in Figure 3.14. In this design, we locate a $5 \mu \mathrm{m}$-radius circular heater with $1 \mu \mathrm{m}$ wide filament (shown in red) right on top of the microring to realize efficient and almost symmetric heating. As the heat generated by feedlines can be negligible, we ignored the feedlines in this simulation.

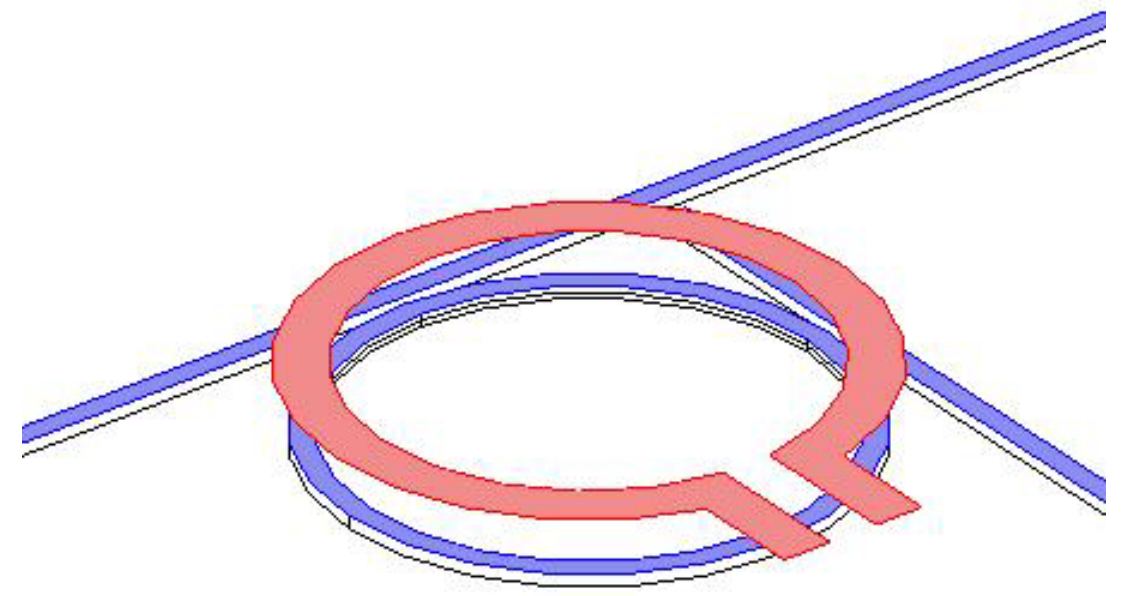

Figure 3.14 Design of ring-shape micro-heater

With an input voltage of $1 \mathrm{~V}$ applied at both ends of the heater, the steady-state simulation results are shown in Figure 3.15, as an analog of Figure 3.11. Approximately 
$1.97 \mathrm{~mW}$ power was generated from the DC Joule heating corresponding to a resistance of $\sim 500 \Omega$. The average temperature change of the microring was $24.99^{\circ} \mathrm{C}$, which is obtained by integrating the temperature change along the microring, The temperature change yields a heating coefficient of $0.079 \mathrm{~mW} /{ }^{\circ} \mathrm{C}$, about $10 \%$ of the S-shape design. That is to say, the same wavelength tuning can be achieved with only $10 \%$ of the heating power using the optimized ring heater. Temperature difference of $\sim 5^{\circ} \mathrm{C}$ between the maximum and minimum temperatures on the ring was observed in the simulation, mainly due to the proximity to the straight waveguides.

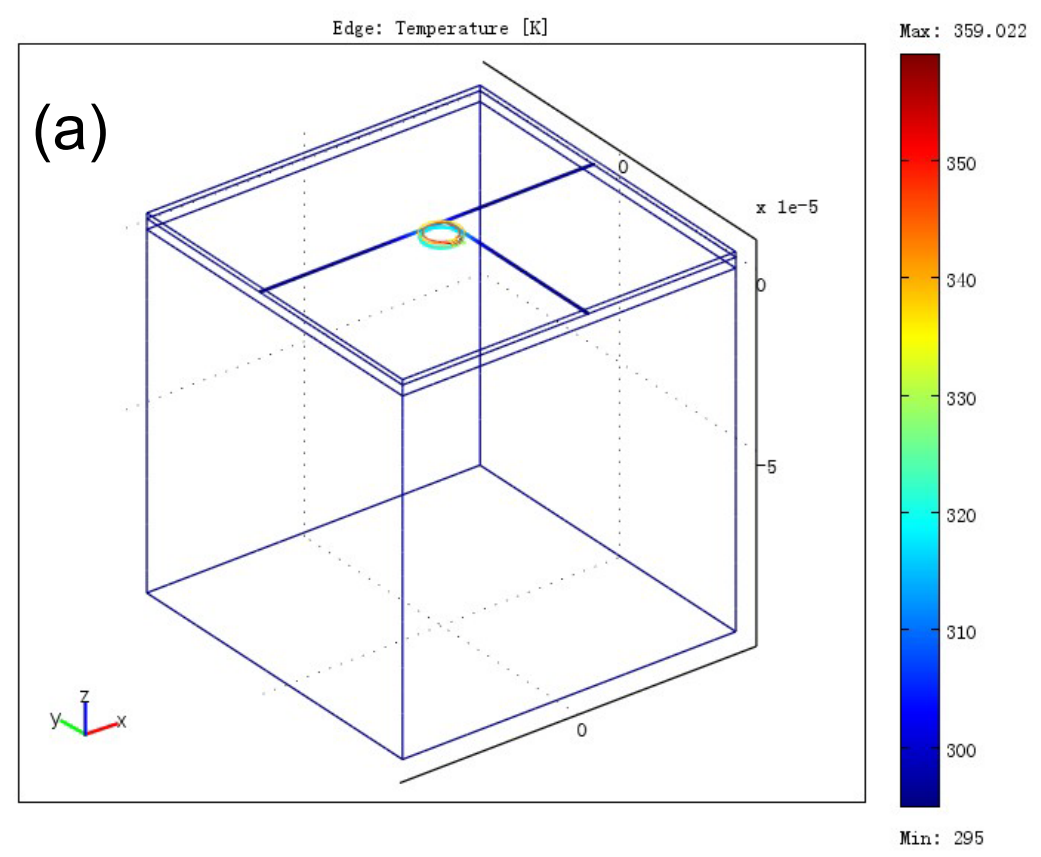



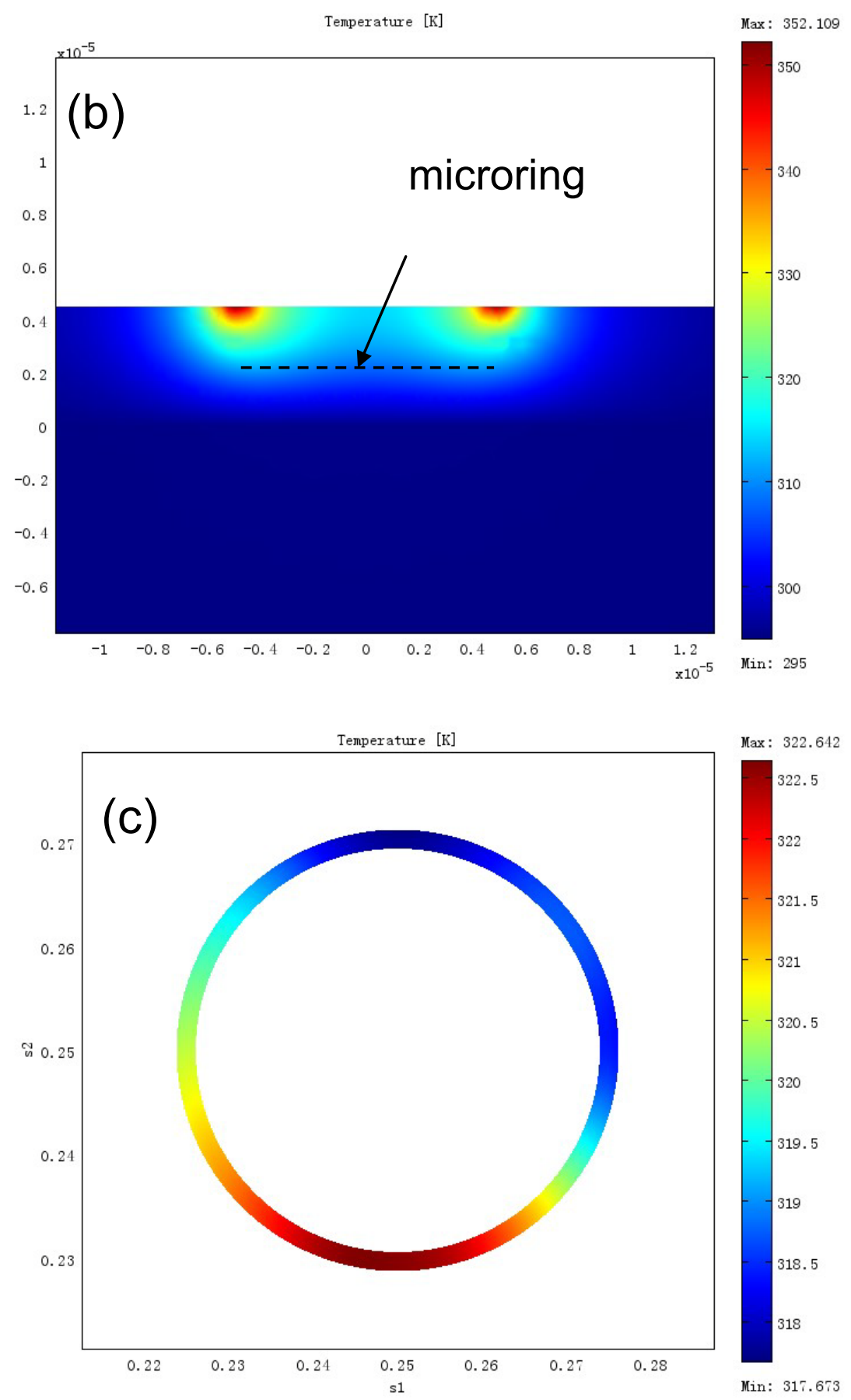

Figure 3.15 Simulated temperature distributions of (a) the whole device (b) x-z plane cross-section at the geometry center of the microring (c) along the microring. All temperatures in Kelvin. The coordinate is shown in (a)

The time response of the optimized heater was simulated also with results shown in Figure 3.16, as an analog of Figure 3.12. The normalized time response of temperature 
change is almost uniform along the microring. Figure 3.17 compares the time responses of average temperature change for both heaters. From the plot, by measuring the time for the average temperature to reach half of its maximum, one can see that the optimized design improves the time response by a factor of two.
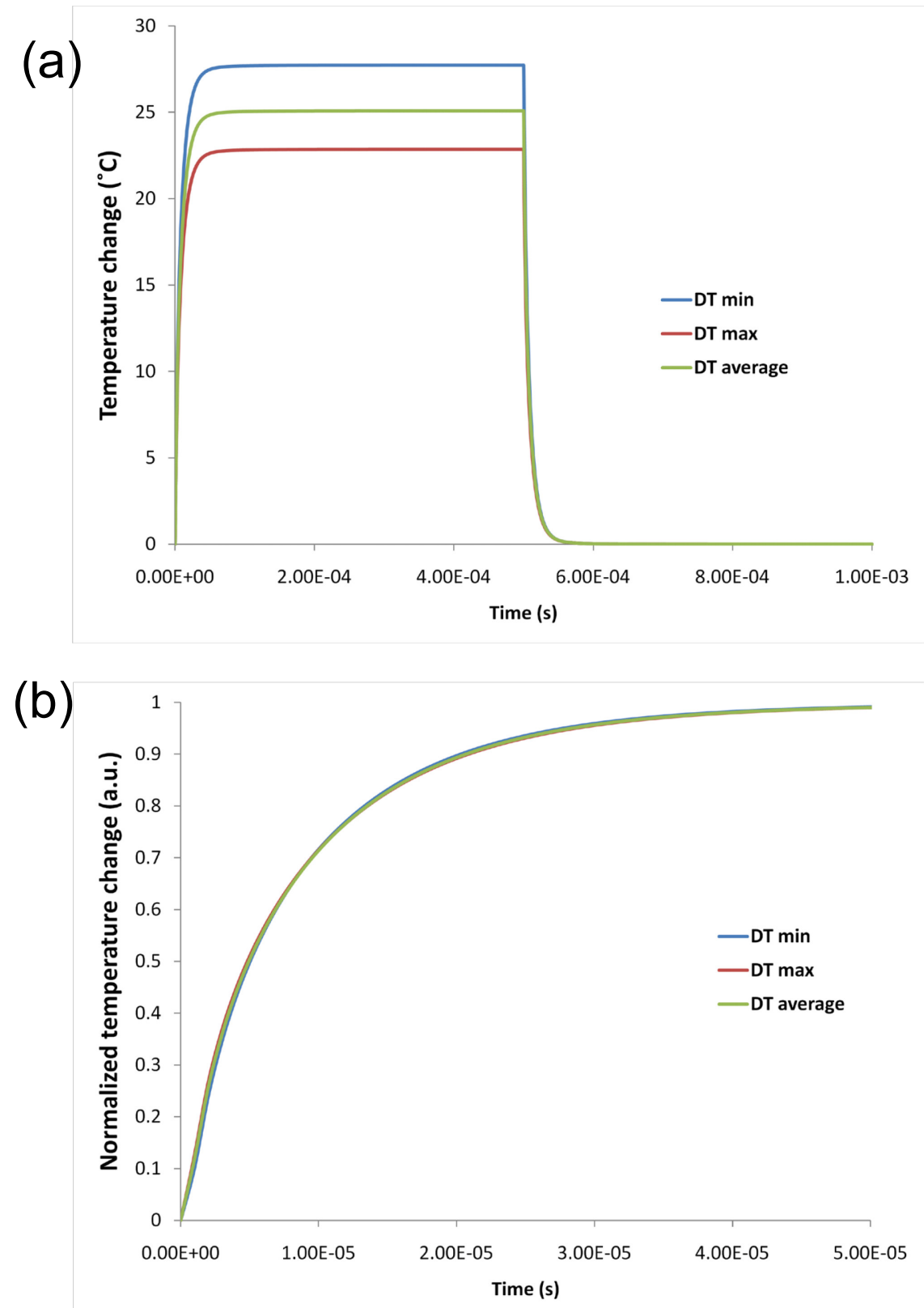

Figure 3.16 (a) Simulated time response of the temperature change of the microring with ring-shape microheater;(b) Normalized simulated time response of the temperature change with ring-shape micro-heater 


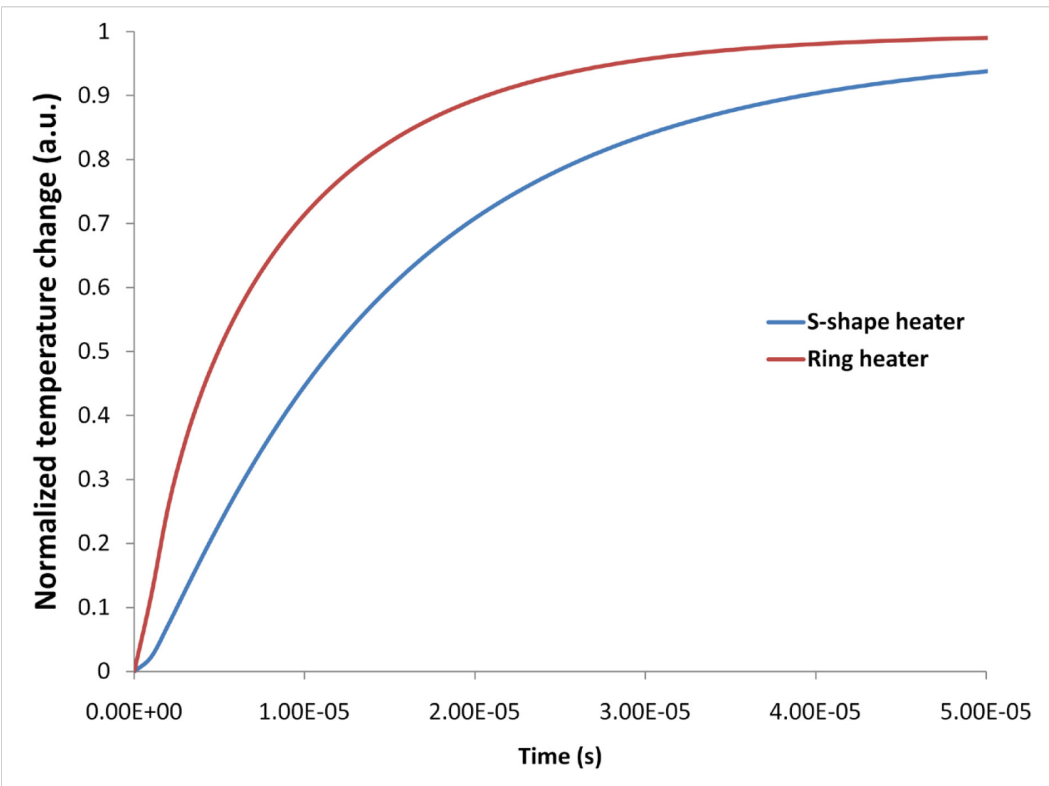

Figure 3.17 Time response of average microring temperature change with the two micro-heater designs

In summary, the optimized design is expected to improve the time response of the heater by a factor of two and at the same time reduce the power consumption by $90 \%$.

\subsection{Thermooptic Tunable Range}

The thermooptic wavelength tuning coefficient of the designed filter at wavelength around $1.55 \mu \mathrm{m}$ was estimated using Equation (2.38): $\frac{\Delta \lambda}{\Delta T}=\frac{\lambda_{0}}{n_{g}} \frac{\partial n_{\text {eff }}}{\partial T}$.The group index $n_{g}$ and effective thermooptic coefficient $\partial n_{e f f} / \partial T$ were obtained by the optical simulations. The group indexes for TE and TM modes used in the calculation were 4.21 and 4.48, which are calculated using Equation (2.20) with the FSR measured from the simulated TE and TM mode spectra in Figure 3.7(a) and (b). To find the effective thermooptic coefficient, a series of BPM simulations were performed to obtain effective index at different temperatures from -20 to $100^{\circ} \mathrm{C}$. A straight waveguide was 
used in the simulations assuming bending effect for the waveguide on microring is negligible. At each temperature, refractive indexes for both silicon and $\mathrm{SiO}_{2}$ are calculated taking into account the thermooptic induced index change. The simulated effective indexes for TE and TM mode were plotted versus temperature in Figure 3.18 (a) and (b). Linear relations were found from the curves with fit slopes of $1.98 \times 10^{-4} /{ }^{\circ} \mathrm{C}$ and $1.73 \times 10^{-4} /{ }^{\circ} \mathrm{C}$ for $\mathrm{TE}$ and $\mathrm{TM}$ mode, respectively. The thermooptic tunability for both modes was found to be $0.075 \mathrm{~nm} /{ }^{\circ} \mathrm{C}$ and $0.063 \mathrm{~nm} /{ }^{\circ} \mathrm{C}$ according to Equation (2.38). Tunability for TM mode is lower compared to that of the TE mode. This is because the optical field for TM mode is less confined in the high thermooptic coefficient area (silicon core) compared to TE mode, which can be observed from Figure 3.1.

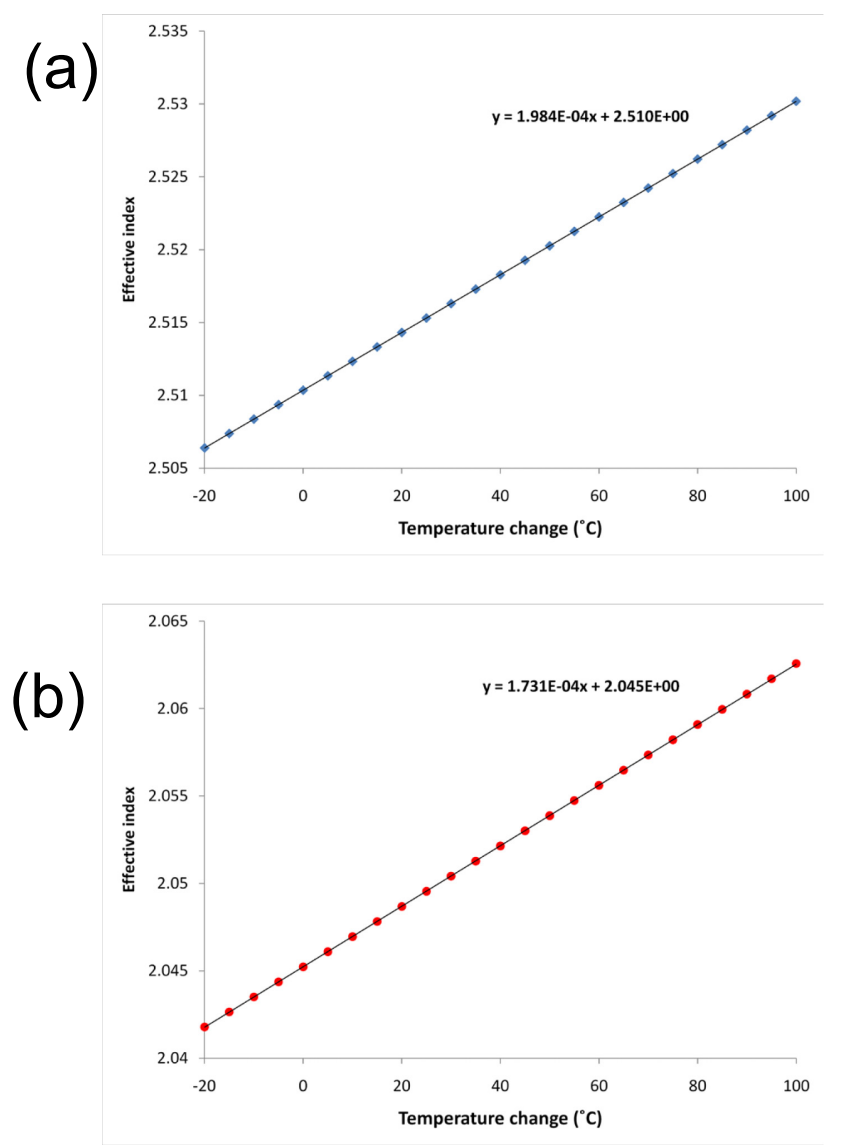

Figure 3.18 Simulated effective index versus temperature for (a) TE mode (b) TM mode 
The tunable range is decided by the maximum microring temperature achievable through heating. It is limited by oxidation, which increases rapidly at high temperatures and electro-migration damage of the heater metal, which happens at high current density and high temperatures. A practical estimation of the maximum heater temperature would be $300^{\circ} \mathrm{C}$ [72] (about $280^{\circ} \mathrm{C}$ temperature change compared to room temperature). The microring temperature is considered to be reduced by approximately a factor of two, i.e., $\sim 140^{\circ} \mathrm{C}$, according to the simulated temperature distribution shown in Figure 3.11(a) (Sshape heater) and Figure 3.15(a) (ring-shape heater). With maximum ring temperature change of $140^{\circ} \mathrm{C}$, wide tunable ranges of $10.5 \mathrm{~nm}$ for TE mode and $8.8 \mathrm{~nm}$ for TM mode were then estimated. 


\section{DEVICE FABRICATION}

\subsection{Fabrication Process Flow}

Using the design described in the previous chapter, we fabricated the thermally tunable microring resonator device. The fabrication is totally compatible with silicon-oninsulator (SOI) technology, which is standard in semiconductor industry. Thus, the device is very promising for photonic integrated circuits.

The fabrication process flow is outlined in Figure 4.1. The fabrication started with a SOITEC SOI wafer with $250 \mathrm{~nm}$ thick Si device layer over a $3 \mu \mathrm{m}$ buried oxide layer. The device layer thickness was chosen according to the designed waveguide dimension. The silicon layer was lightly P-doped (Boron) with nominal resistivity of $13.5-22.5 \Omega \mathrm{cm}$. The waveguide pattern was generated by a LeicaVB6-HR $100 \mathrm{kV}$ ebeam lithography system at $1 \mathrm{nA}$ exposure current with $5 \mathrm{~nm}$ beam step. A negative ebeam resist named hydrogen silsesquioxane (HSQ) was used. The ebeam lithography process and fabrication considerations will be discussed in detail later in this chapter. The resist was spun at 2000 $\mathrm{rpm}$ and baked at $200^{\circ} \mathrm{C}$ for $2 \mathrm{~min}$. A HSQ film of $\sim 120 \mathrm{~nm}$ thickness was generated after the bake. The dose we used to write the waveguide and rings is $1100 \mu \mathrm{C} / \mathrm{cm}^{2}$. The waveguides are finished with nanotapers which were assigned higher doses from $1300 \mu \mathrm{C} / \mathrm{cm}^{2}$ to $2300 \mu \mathrm{C} / \mathrm{cm}^{2}$. Post-exposure bake was carried out at 200 for two minutes. Development was performed in MIF-300 developer for two minutes and rinsed in deionized water. Samples were characterized by SEM after this step. A SEM image of a waveguide- ring coupling region is shown in Figure 4.2(a). The waveguide pattern was

then transferred to silicon by $\mathrm{Cl}_{2}$ reactive ion etching (RIE) process using a PlasmaTherm 
770 ICP etching tool. The RIE process etched the unpatterned area, leaving a vertical sidewall on the edge of the patterned area, which forms the strip waveguide. The remaining HSQ layer on top of the waveguide does not need to be stripped off because the optical property of the HSQ is very close to $\mathrm{SiO}_{2}$, which will be the cladding of the waveguide later. The etched microring resonator SEM picture is shown in Figure 4.2(b). An optional LPCVD $\mathrm{Si}_{3} \mathrm{~N}_{4}$ process was done on the etched device after a MOS clean process required for the LPCVD process. About $30 \mathrm{~nm}$ thick $\mathrm{Si}_{3} \mathrm{~N}_{4}$ layer was deposited after the process, serving as a protective layer for prospective future development of the device that need to remove upper cladding by buffered oxide etch (BOE). Considering the very thin thickness of the silicon nitride layer and the significant lower refractive index of about 2.02 [63] compared to 3.48 of the silicon core, it is not expected to affect the optical property of the device significantly. The upper $\mathrm{SiO}_{2}$ cladding was deposited using PECVD with a measured thickness of $1.4 \mu \mathrm{m}$, close to the designed thickness of $1.5 \mu \mathrm{m}$. The SEM picture was then taken as shown in Figure 4.2(c). 
a) SOI substrate

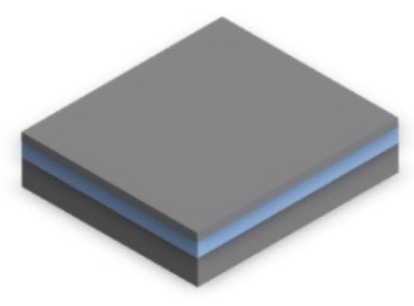

d) RIE etch Silicon

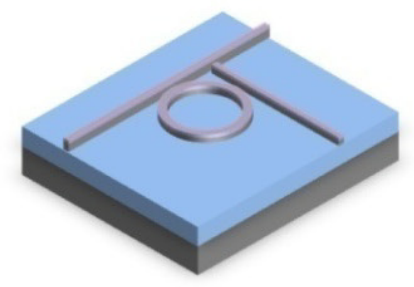

g) Spin on photo resist

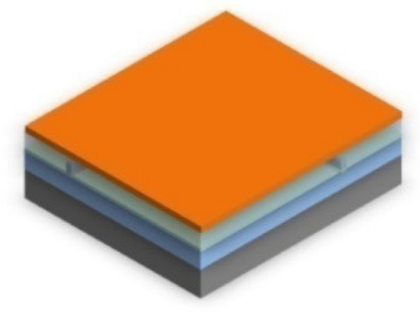

j) Micro-heater liftoff

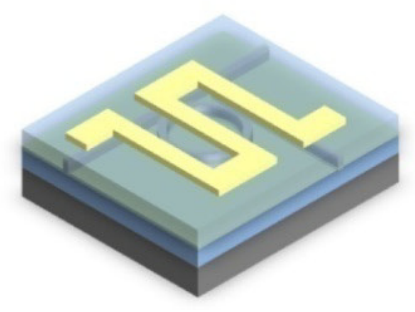

b) Spin-on HSQ

(Ebeam resist)

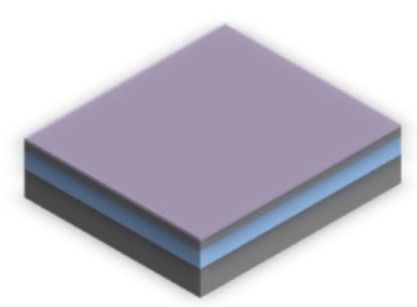

e) LPCVD Si3N4 (optional)

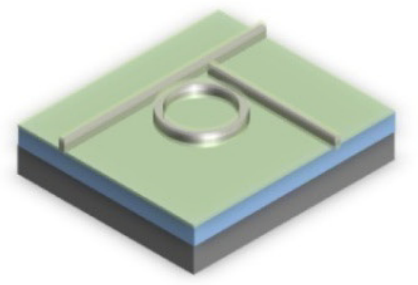

h) Pattern micro-heater using Photolithography

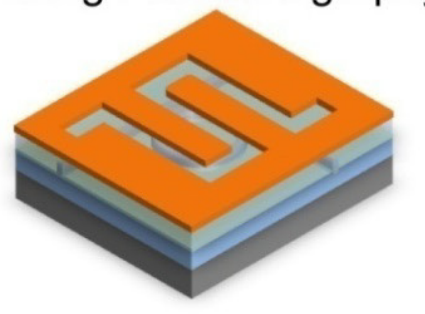

k) Ti/Au feedline liftoff

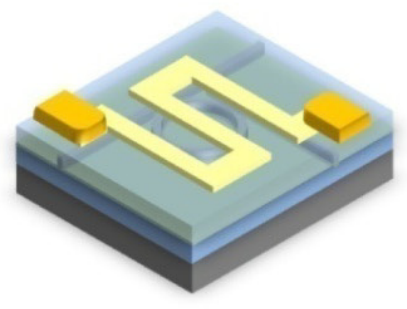

c) Pattern waveguide using ebeam lithography

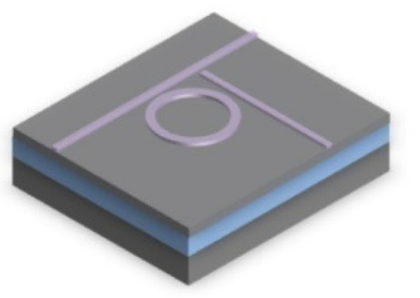

f) PECVD SiO2 cladding

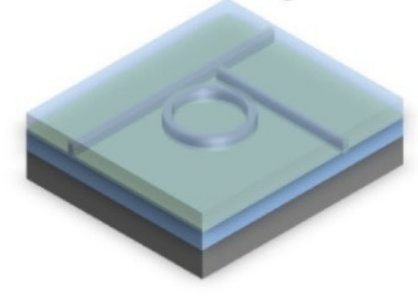

i) $\mathrm{Ni}-\mathrm{Cr}$ evaporation

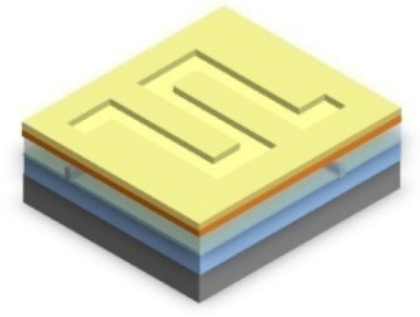

Figure 4.1 Fabrication process flow of the tunable microring resonator device 

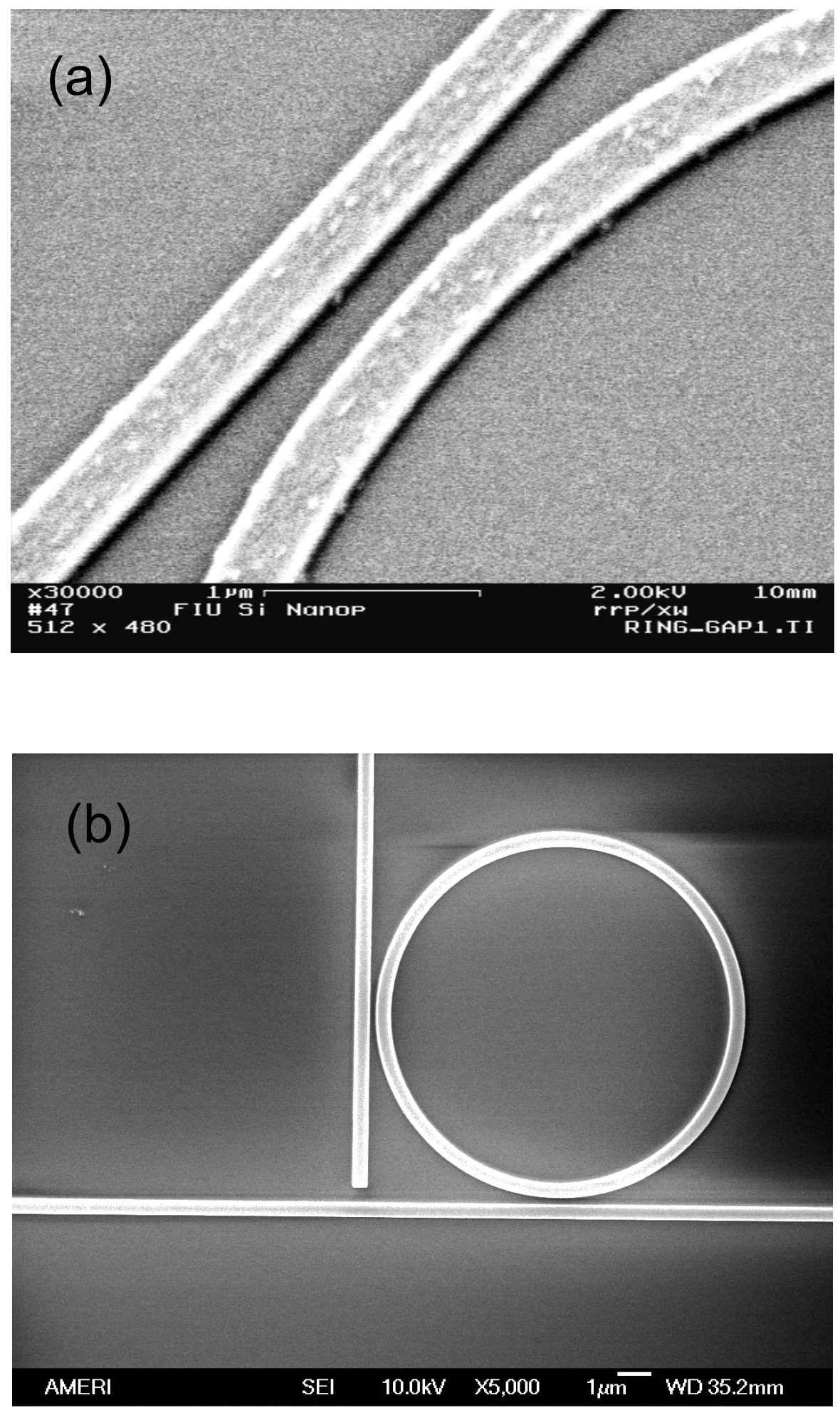


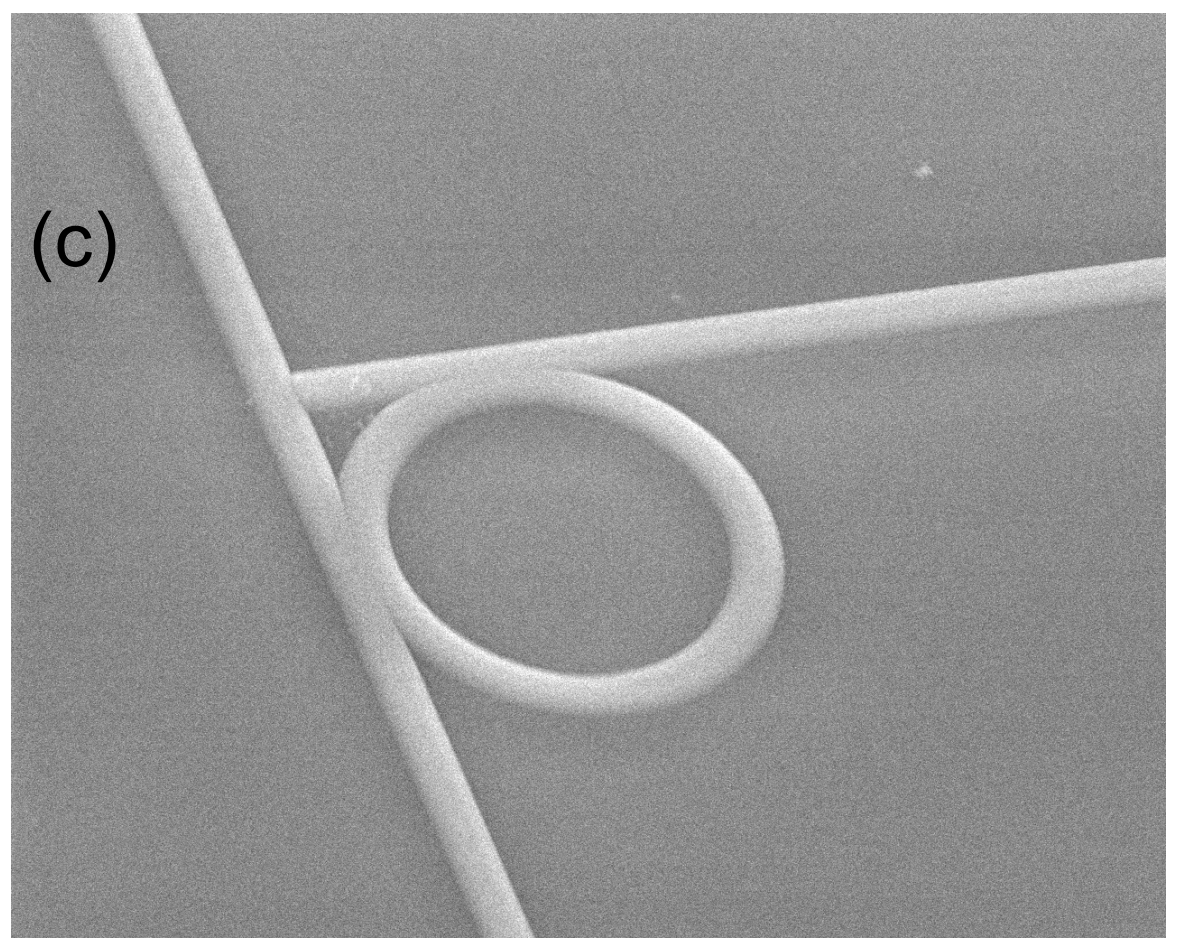

Figure 4.2 SEM pictures of (a) patterned HSQ resist at gap between microring and straight waveguide (b) microring resonator device after silicon etch (c) microring resonator device with $\mathrm{SiO}_{2}$ cladding

The micro-heater and its feedlines were generated separately on the $\mathrm{SiO}_{2}$ cladding using liftoff technique. As the dimension of the micro-heater and the feedlines are in $\mu \mathrm{m}$ size, photolithography was used for pattern generation. In order to form an undercut profile which is critical for the liftoff process, double-layer resist was used. First, a layer of LOR 10A resist was spun on the $\mathrm{SiO}_{2}$ cladding at $4000 \mathrm{rpm}$ and baked at $17 \mathrm{Cl}$ for five minutes. This step results in a LOR resist layer of about $1 \mu \mathrm{m}$ in thickness. The LOR layer thickness is much bigger than the metal thickness to be deposited, making the liftoff process easy. Then, a S1813 photo resist was spun on top of the LOR resist. The spin speed is $4000 \mathrm{rpm}$ and post bake was at $115^{\circ} \mathrm{C}$ for one minute. The thickness of the $\mathrm{S} 1813$ resist was estimated to be $1.3 \mu \mathrm{m}$. Photolithography was done using a HTG contact aligner with wavelength of $365-405 \mathrm{~nm}$ broadband illumination. Intensity of the 
illumination was measured as $\sim 10 \mathrm{~mW} / \mathrm{cm}^{2}$ at $365 \mathrm{~nm}$ and $\sim 20 \mathrm{~mW} / \mathrm{cm}^{2}$ at $405 \mathrm{~nm}$. The heater pattern was aligned to the waveguide pattern using alignment marks designed on both patterns. The sample was developed using MIF300 developer after a 6-second exposure. A 100nm-thick film of Ni-Cr was then deposited using a CVC 4500 evaporator with a deposition rate of 3-5 $\AA / \mathrm{sec}$ in order to form a uniform metal layer. The liftoff was performed using LOR remover. The Ti/Au feedlines were deposited using similar liftoff process. However, the film was deposited by sputtering using an AJA ATC1600 sputtering machine. A thin layer of $\operatorname{Ti}(\sim 5 \mathrm{~nm})$ was sputtered before the Au layer in order to promote the adhesion between $\mathrm{Au}$ and $\mathrm{SiO}_{2}$. The $\mathrm{Au}$ layer was measured to be about $120 \mathrm{~nm}$. The feedline pattern was then aligned to the heater pattern. A top-view microscopic picture of a fabricated device is shown in Figure 4.3.

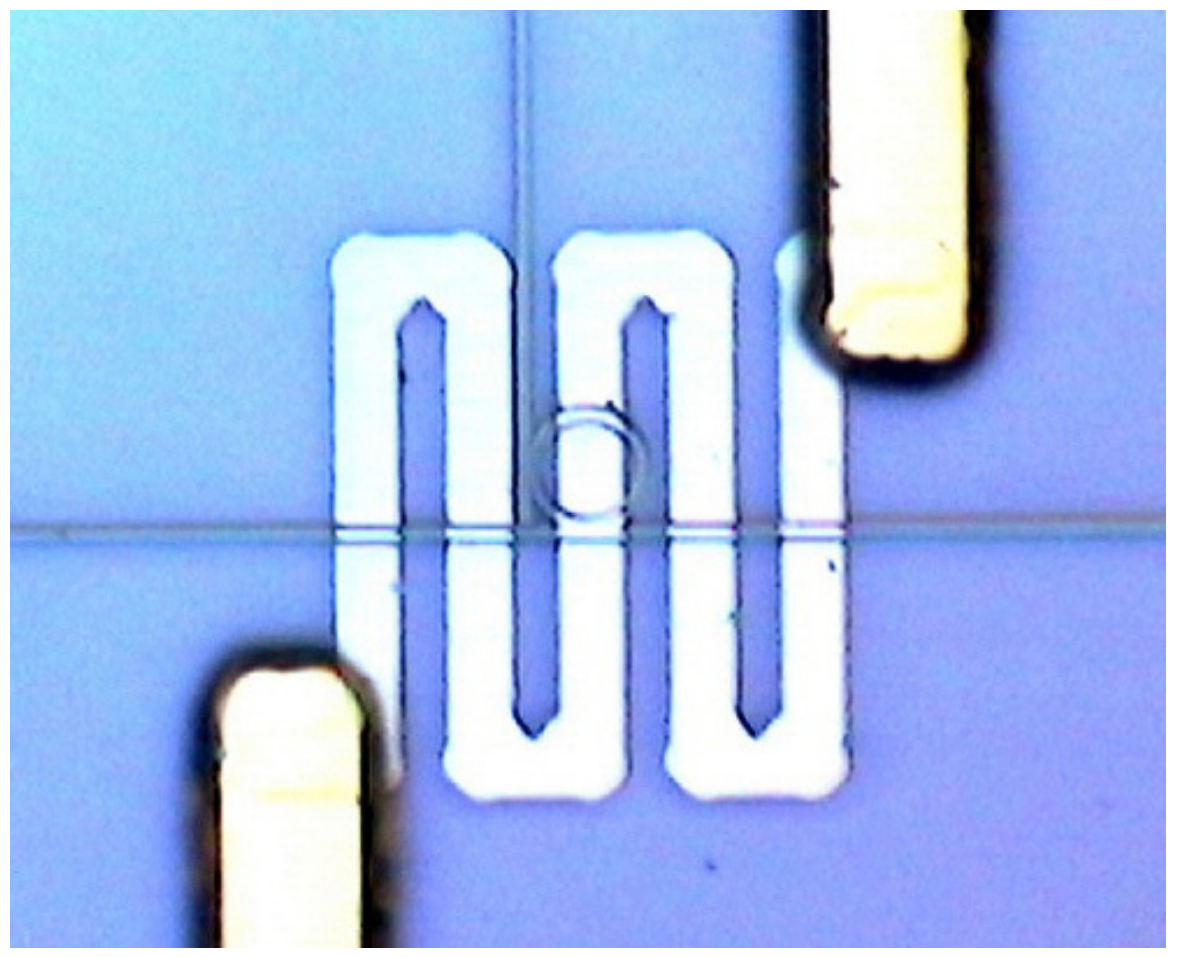

Figure 4.3. Top-view microscopic image of micro-heater on top of Si based microring resonator 


\subsection{Electron-beam Lithography}

Electron beam lithography (often called "ebeam lithography") is a technique of scanning a beam of electrons across a surface coated with resist and selectively removing either exposed or non-exposed area of the resist depending on the resist type. Compared to photolithography, it can create smaller structures in the resist because it beats the diffraction limit of light. It is capable of making features in the nanometer regime. On the other hand, the key limitation of ebeam lithography is throughput. A very long exposure time is often needed for a common sample. The technique has found wide usage in photomask-making, low-volume production of semiconductor components, and research and development.

Since patterning of our designed device based on 450nm-width waveguide with nanometer precision is beyond the capability of the conventional photolithography tools, we decided to use ebeam lithography for the waveguide patterning. The Leica VB6-HR system for patterning the optical layer of the device is an advanced ebeam lithography system capable of reproducibly achieving feature sizes less than $30 \mathrm{~nm}$. The system has a precision stage with stage positioning monitored by a laser interferometer with $\lambda / 1024=$ $0.6 \mathrm{~nm}$ precision. It makes possible exposing pattern with area larger than the size of the electron beam writing field (usually a square with side length a few hundred of micrometer) by stitching (tiling writing fields exactly against each other) and pattern overlay (aligning a pattern to a previously made one). The VB6 is also equipped with a real-time laser height sensor for dynamic field size corrections and dynamic focus/astigmatism corrections. 
XR-1541 HSQ 6\% was used as the ebeam resist. Unlike the most popular ebeam risist PMMA, the HSQ resist is a negative resist. The choice of negative resist is made according to the nature of our pattern, in which, the total area of waveguide to be exposed took only less than $1 \%$ of the total sample area. As the exposure time is proportional to the exposed area, we only expose the waveguide region in order to reduce the exposure time. Thus a negative resist is chosen (the exposed area stays).

The VB6 system can align different patterns. Like in photolithography, alignment marks are always needed on the pre-existing pattern. An electron microscopic system was used to "see" the alignment marks by detecting the backscattered electrons from the substrate. The VB6 can establish a coordinate system based on alignment marks detected on the wafer and precisely place a pattern with respect to preexisting patterns. Alignment marks are recommended to be designed far from the device area because the mark detecting process will expose the area around it.

\subsection{Sample Preparation and Packaging}

The fabricated samples need further processing before they are ready to be characterized. Integrated photonic circuits enables optical devices with multiple functions integrated in a very small area of substrate, usually with dimension of centimeters or millimeters and sometimes even micrometers. The small footprint enables us to put tens or even hundreds of devices on a single wafer, which greatly increases the throughput. However, preparation and packaging need to be done before one can use or test the device. Nowadays, it is one of the major challenges in integrated photonics. 
The way we test the device is by coupling light in and out via tapered fiber. This configuration requires that the input and output waveguides end at the edge of the chip and the facet need to have a good flatness to avoid big losses due to light scattering. As mentioned previously, nanotapers [44] were used at the end of the waveguide to increase coupling efficiency by tapering down the width of the waveguide from $450 \mathrm{~nm}$ to $120 \mathrm{~nm}$. Figure 4.4 shows a microscopic picture of a fabricated nanotaper.

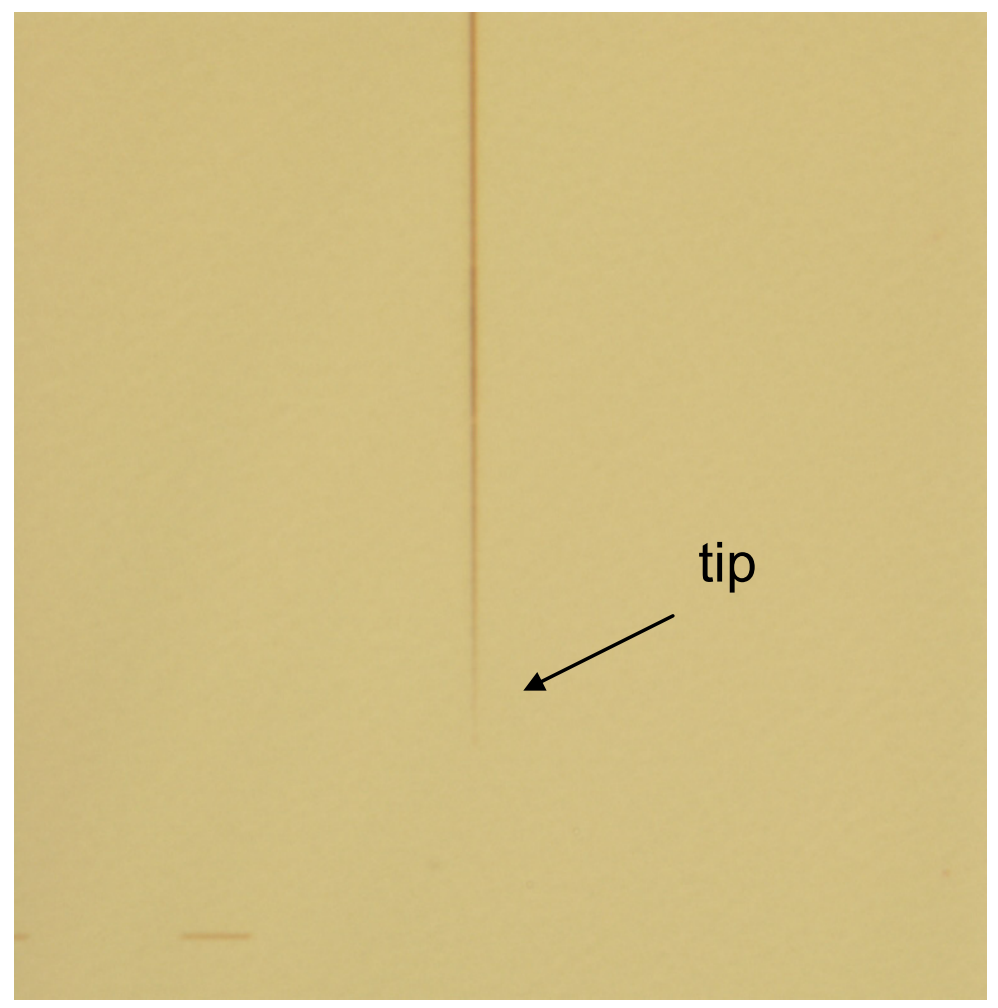

Figure 4.4 Microscopic picture of a fabricated nanotaper

\subsubsection{Dicing}

To prepare the sample for optical characterization, the fabricated substrate was diced into small samples first using a K\&S 7100 dicing saw. The sample was glued on a film held firmly on the system. The dicing saw uses a vision system to detect marks on the sample and establish a coordinate system for the dicing pattern similar to the 
alignment system for lithography. A rotating blade then makes cuts along $\mathrm{X}$ and $\mathrm{Y}$ direction at positions defined by the dicing pattern. The edges after dicing are with roughness of about $50 \mu \mathrm{m}$ measured by microscopic inspection. Our samples are cut $150 \mu \mathrm{m}$ away from the end of the nanotapers, allowing enough room to avoid the nanotaper from damaging by the blade.

\subsubsection{Polishing}

Like most of the optical components, ultra-flat and clean optical interfaces are needed to avoid high light-scattering loss. Polishing is a common approach to prepare the optical interfaces. For our device, aside from the requirement for the facets, precise control of the positions and orientations of the interface (edge of the chips) is critical because the nanotaper tips need to be located at the edges of the chip and perpendicular to the waveguide in order to couple light in and out of the waveguide-based device efficiently. This is a challenge for polishing. The samples were polished manually using a rotating plate equipped with diamond lapping films. To manipulate the samples to be polished, the samples were glued on a holder by crystal bond. A lapping film with the larger diamond grit size removes materials faster, however, it will yield a rougher surface. In order to achieve good facet quality and efficiency, a procedure was developed using a multi-step polishing process. Samples were polished first with a rough lapping film (with large diamond grit size), followed by finer and finer films to remove the rough edge left by the last step. The finest film we use was with diamond grid size of $0.05 \mu \mathrm{m}$, which left a very shiny facet. To have the polished facets end at the tip of the nanotapers with precision of a few tens of micrometers, the removal thickness of each step need to 
be planned. We usually set the removal thickness of each step as three times of the diamond grid size for the last step to make sure the rougher surface from the last step was completely removed. Frequent inspection under microscope is crucial in monitoring the orientation and distance to the target plane. Over-polishing is unacceptable as it will damage the nanotaper. In order to facilitate the polishing process, polishing marks and guidelines were designed and patterned together with the waveguide. A sample polishing pattern is shown in Figure 4.5.

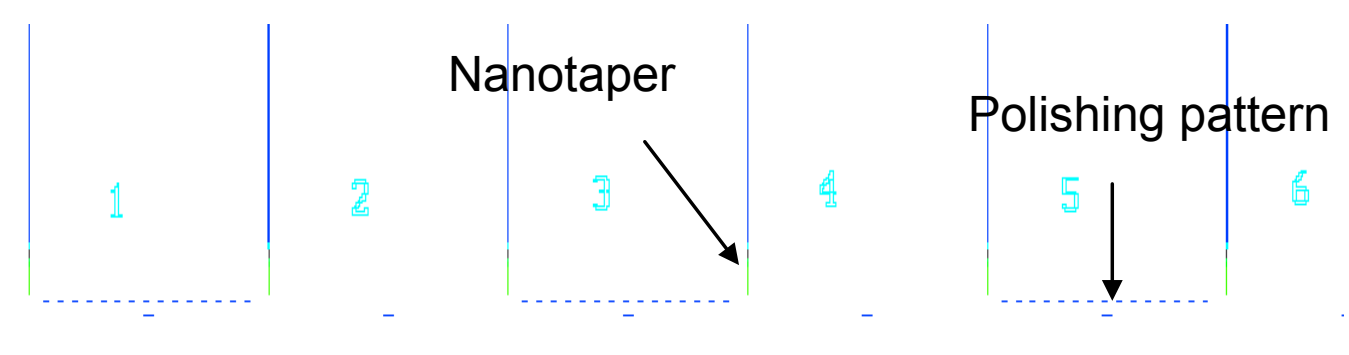

Figure 4.5 Design of a polishing pattern

\subsubsection{Wire bonding}

For an application discussed in Chap.VI, while many wavelength-tunable filters need to work simultaneously and independently, connecting multiple conventional electrical probes to the millimeter size sample is impractical. Wire bonding is a technology developed and widely used in micro-electronics to solve this problem. With this technology, a very thin metal wire with thickness of tens of micrometer or even smaller can be used to make electrical connections between electrode pads. 
To simultaneously connect multiple microheaters, a special mount as the bonding platform was designed with a printed circuit board (PCB) containing the interface circuits for collecting micro-heaters to the electrical source. A polished sample was fixed on the holder. Thermal silver epoxy was used to bond fine stripped magnet wires with a diameter of $45 \mu \mathrm{m}$ to the PCB and the electrode pads on the sample. A microscopic picture of an electrode pad was shown in Figure 4.6. The bonded device with mount will be shown later in this work in Figure 6.2. The wire bonding was performed by our research partner H.Y.Ng at the University of Miami.

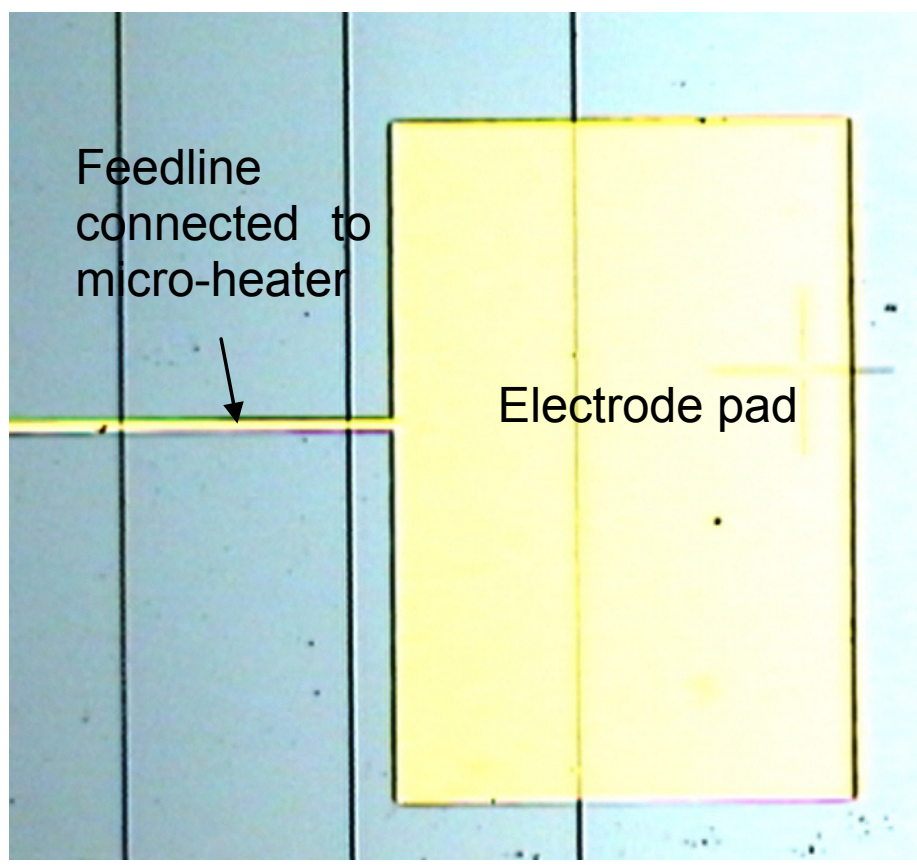

Figure 4.6 Microscopic picture of electrode pad connected to a micro-heater feedline. The dark lines are silicon waveguide which is under the metal micro-heater and feedline/pad layer separated by the upper cladding of $1.4 \mu \mathrm{m}$. 


\section{DEVICE CHARACTERIZATION}

\subsection{Characterization Setup}

The performance of the fabricated tunable microring resonator device was tested using our optical characterization setup. The block diagram of the setup is shown in Figure 5.1. To characterize the optical property of the device, light needs to be launched into the input waveguide and collected from the output waveguide. For the input part, we use a polarization-maintaining tapered fiber to couple light from the light source to the input waveguide ended with a nanotaper [44] as described before. As for the output, either a tapered fiber or an objective lens could be used in different applications. The tradeoff between them will be discussed later in this chapter. Two Thorlab NanoMAX manual XYZ stages with piezo were used to align the input and output tapered fiber/objective lens to the input and output waveguide on the sample. The fine alignment was done using the internal piezos of the stage, which can be adjusted without touch with $20 \mathrm{~nm}$ resolution for all 3 axes. A long working distance 20x objective lens was mounted on top of the sample movable in all 3 axes for rough alignment monitoring. Either a RGB camera or an IR camera can be used for imaging with help of the objective. The long working distance (over $20 \mathrm{~mm}$ ) between the objective lens and the sample enables direct electrical probing without blockage by the lens. The whole setup was mounted on an optical table with vibration isolation and covered by an enclosure to avoid heat exchange of the sample with the ambient environment due to the air flow. 


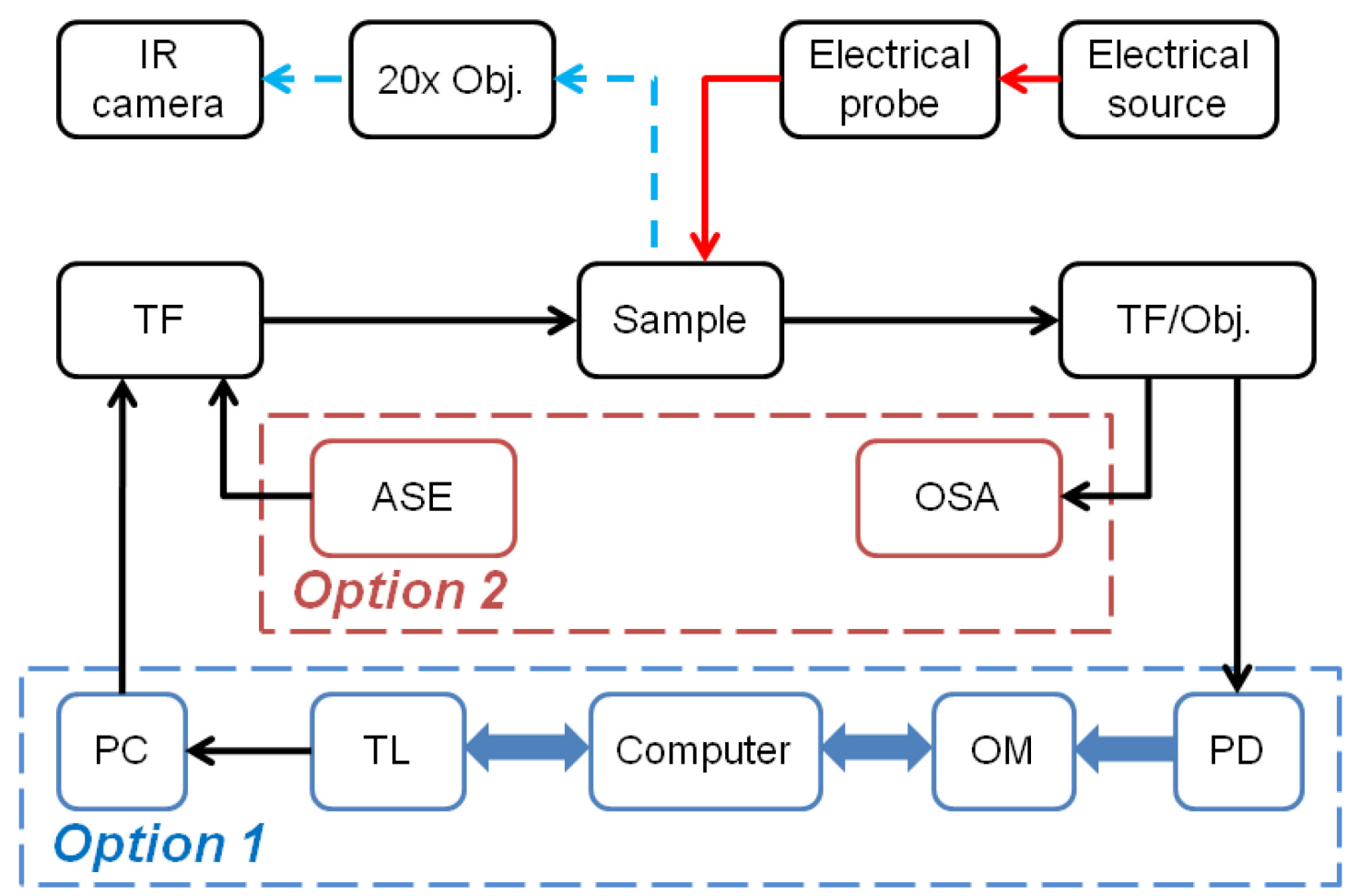

Figure 5.1 Experimental setup block diagram for device characterization. Obj--objective lens, TF -tapered fiber, ASE- amplified spontaneous emission source, OSA-optical spectrum analyzer, PC-polarization controller, TL-wavelength tunable laser, OM-optical meter, PD-photodiode.

Photographs of the setup are shown in Figure 5.2. It includes the input and output stage, the top-view system with objective lens and a RGB camera, the sample mount for testing, all in an enclosure. A close-up view shows the input and output tapered fibers and the electrical probes for micro-heater current injection. All instruments are mounted on the shelf over the optical table to avoid vibration.

The transmitted power can be collected using a tapered fiber or an objective lens. Either of them can be aligned to the output waveguide using the XYZ stage mounted with different fixtures. The objective lens makes possible imaging of the output facet of the sample by camera, which gives the information of the power distribution at the output instead of the total output power. However, the tapered fiber makes the alignment easier 
given the micron-sized tip and provides convenient connection to other instrument by the FC connector at the other end of the fiber.
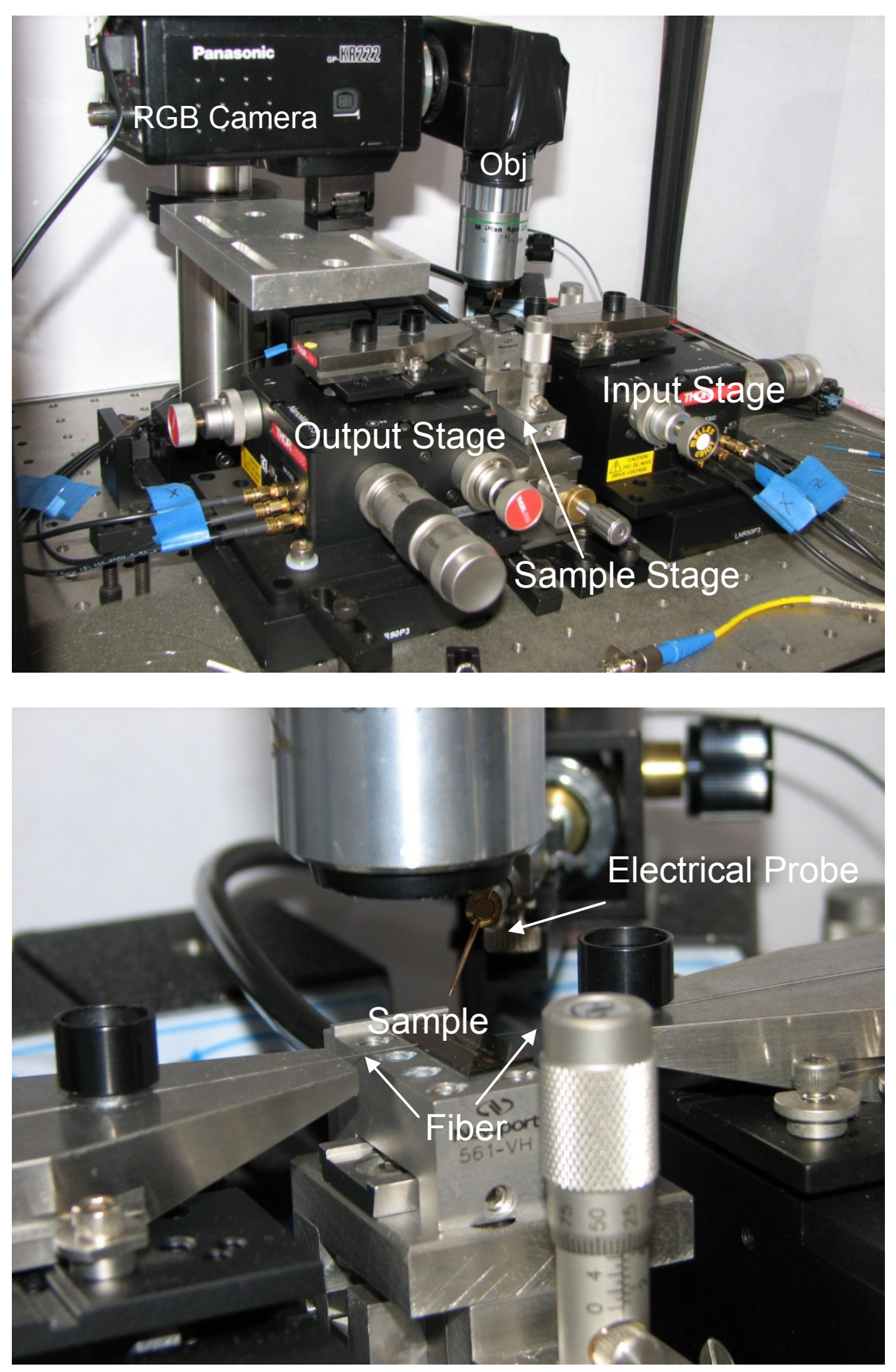

Figure 5.2 Pictures of the characterization setup 
The spectrum response of the device under test was often of interest. With our testing setup, it can be obtained using either a tunable laser (ANDO AQ4321D) or a broadband amplified spontaneous emission (ASE) source (ANDO AQ4310) shown as Option 1 and Option 2 in Figure 5.1, respectively. For Option 2, the output from the device was connected to an optical spectrum analyzer (OSA) (ANDO AQ6317B). Both light sources cover the target working wavelength of our device of around $1.55 \mu \mathrm{m}$, the standard wavelength band used in fiber communication. Option 2 is usually used for quick measurements with low resolution. For high-resolution characterization, Option 1 was configured. The tunable laser is a polarized light source with output wavelength confined within $1 \mathrm{pm}$. The output wavelength can be tuned in range of $1520-1620 \mathrm{~nm}$ with $1 \mathrm{pm}$ resolution. A polarization controller was connected to control the input polarization state of the sample. In this configuration, the transmitted light was detected by a calibrated photo-detector (Newport 818-IR) and the optical power was read by a Newport 1835-C optical meter. While maintaining the output power of the tunable laser, the transmitted optical spectrum response of the device under test was measured by sweeping the output wavelength of the tunable laser and recording the detected transmitted power for each wavelength. Both tunable laser and optical meter were controlled by a computer through GPIB ports. A LabView program was written to control the tunable laser and the optical meter while recording data. 


\subsection{Optical Spectral Response Measurements and Analysis}

The optical properties of our fabricated tunable microring resonators were characterized without heater current injection first. At room temperature of $2 \mathbb{C}$, drop port spectra were measured with input polarization set to TE (E-field parallel to device plane) and TM (E-field perpendicular to device plane) using the tunable laser configuration in Figure 5.1 (Option 1). Figure 5.3 plots the drop port transmitted TE and TM spectra of one microring in wavelength range $1520 \mathrm{~nm}-1620 \mathrm{~nm}$. Each spectrum was normalized to its maximum power. With the data, the values of resonance wavelengths, FSR and FWHM for TE and TM modes were extracted and listed in Table 5.1 and Table 5.2, respectively.

Table 5.1 Summary of TE mode parameters

\begin{tabular}{|c|c|c|c|c|c|}
\hline Resonance (nm) & 1537.46 & 1555.31 & 1573.70 & 1592.43 & 1611.59 \\
\hline FSR (nm) & & 17.85 & 18.39 & 18.73 & 19.16 \\
\hline FWHM (nm) & 0.10 & 0.20 & 0.14 & 0.17 & 0.18 \\
\hline $\mathrm{Ng}$ & & 4.26 & 4.24 & 4.26 & 4.26 \\
\hline $\mathrm{Q}$ & 15374.60 & 7776.55 & 11240.71 & 9367.24 & 8953.28 \\
\hline
\end{tabular}

Table 5.2 TM Summary of TM mode parameters

\begin{tabular}{|c|c|c|c|c|c|}
\hline Resonance (nm) & 1533.81 & 1550.32 & 1568.00 & 1586.84 & 1605.16 \\
\hline FSR (nm) & & 16.51 & 17.68 & 18.84 & 18.32 \\
\hline FWHM (nm) & 0.71 & 0.81 & 1.26 & 1.24 & 1.98 \\
\hline Ng & & 4.58 & 4.38 & 4.20 & 4.43 \\
\hline Q & 2160.30 & 1913.98 & 1244.44 & 1279.71 & 810.69 \\
\hline
\end{tabular}




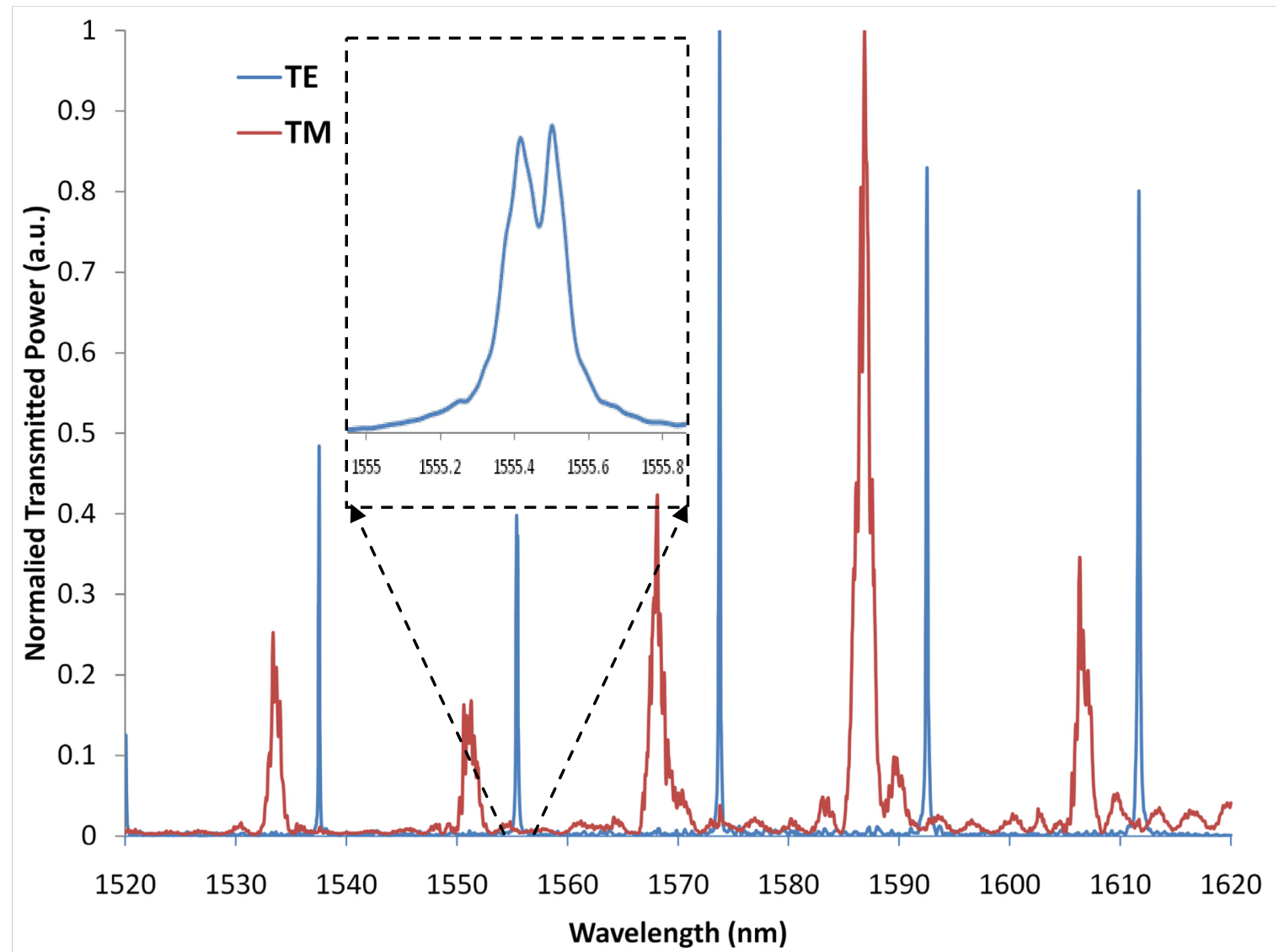

Figure 5.3 Measured drop port transmission spectra of a fabricated tunable microring resonator at room temperature of $22^{\circ} \mathrm{C}$ with TE and TM input polarization. Inset shows the resonance spectrum of peak around $1555 \mathrm{~nm}$ with higher resolution.

Measurements were repeated for the other eight microring resonators with identical design on the same sample. The group index and quality factor for all nine measured microring resonators were calculated from the extracted parameters using Equation (2.20) and (2.30), respectively. Figure 5.4 (a) and (b) plot the measured group index and simulated group index using FDTD and BPM for TE and TM modes, respectively. The FDTD group index was extracted from Figure 3.7. And the BPM one was calculated by $n_{g}=n_{e f f}-\lambda\left(\frac{d n_{e f f}}{d \lambda}\right)[64]$. The measured group indexes are in agreement with the simulation results. The extracted Qs for TE and TM modes are ploted in Figure 5.5 comparing them with the simulated Qs. The simulated Qs are calculated 
from the coupling efficiency shown in Figure 3.4 using Equation (2.30) and (2.28). One can observe that the measured Qs are in agreement with the simulation as well, indicating a low loss in the microring. In Figure 5.5 (a), a much lower Q at $\sim 1555 \mathrm{~nm}$ is due to its double resonance shown in the inset of Figure 5.3. The double-peak is a signature of contra-directional coupling in the ring resonator. Roughness, surface scattering defects and shape non-idealities can couple the two counter-propagating modes [46]. The higher deviations for measured group indexes and Qs of the TM mode shown in Figure 5.4 (b) and 5.5 (b) are due to the larger measurement errors for resonance wavelength and FWHM because of the broad linewidth of TM resonance.

The optical properties of the microring resonator were compared to other similar devices reported [78-80], showing excellent agreement. Table 5.3 summarizes the results from the literature. All these devices were fabricated on SOI and have the same microring radius of $5 \mu \mathrm{m}$. All measurements are taken with TE polarization input. The waveguide dimension are slightly different, however, and the width/height ratio are all around two. The gap between microring and the straight waveguide ranges from $200 \mathrm{~nm}$ to $450 \mathrm{~nm}$. The measured FSR are about $18 \mathrm{~nm}$ except in Reference [80] where wider microring waveguide are used. The resonance FWHMs are all in the range of $0.1-0.2 \mathrm{~nm}$ except the one with large gap in [80]. In that device, the $\mathrm{Q}$ is higher due to the much smaller coupling coefficient with the larger gap. 

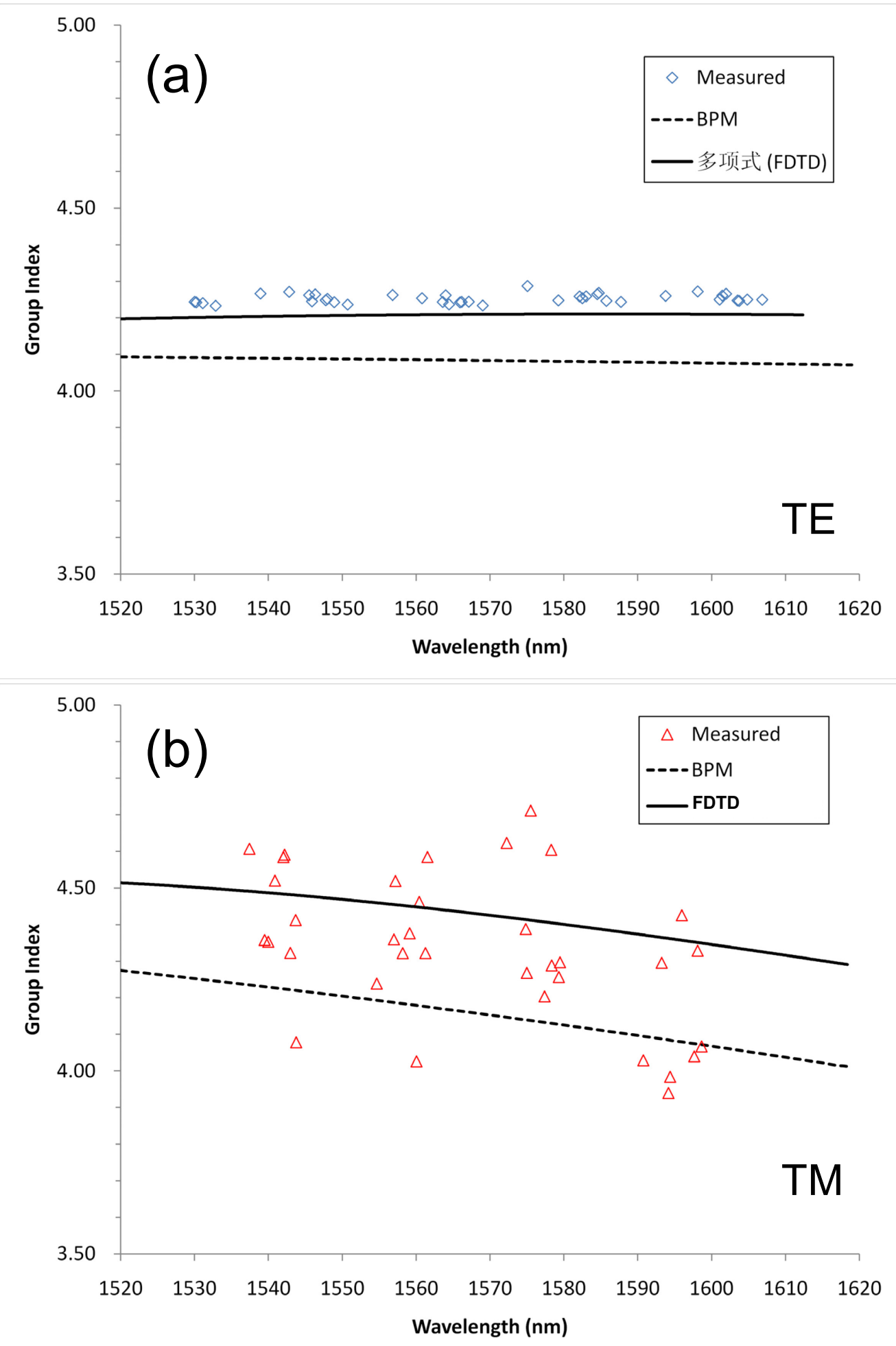

Figure 5.4 Measured group indices (dots) for (a) TE and (b) TM polarization compared with simulated calculations based on FDTD (solid), beam-propagation (dashed). 

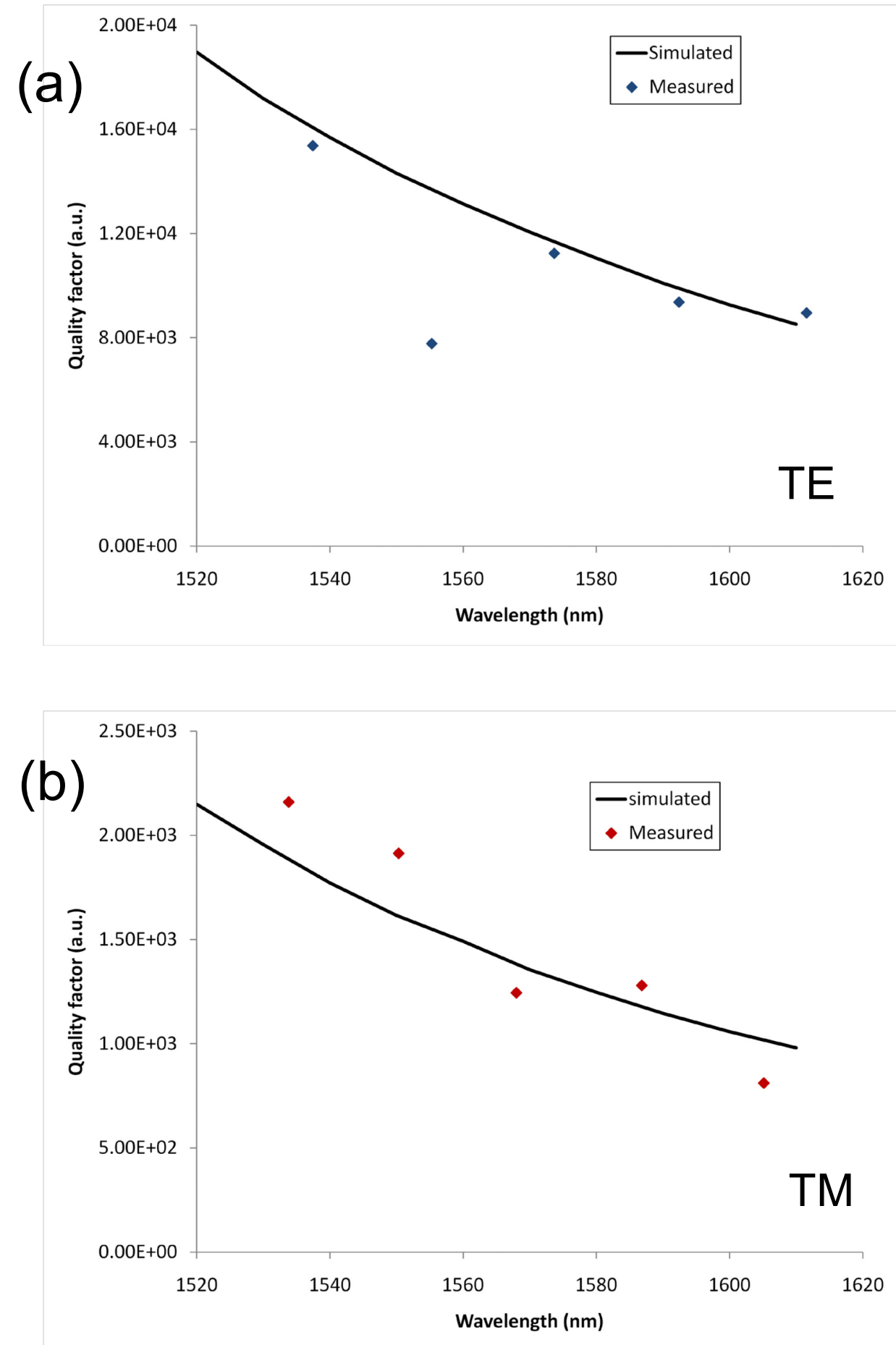

Figure 5.5 Measured quality factor (dots) for (a) TE and (b) TM polarization and compared with simulated (solid). 
Table 5.3 Comparison of optical properties measured in this work and in other literatures

\begin{tabular}{|c|c|c|c|c|c|c|c|}
\hline Reference & $\begin{array}{c}\text { Waveguide } \\
\text { Height } \\
(\mathrm{nm})\end{array}$ & $\begin{array}{c}\text { Waveguide } \\
\text { Width } \\
(\mathrm{nm})\end{array}$ & $\begin{array}{c}\text { Ring } \\
\text { Radius } \\
(\mu \mathrm{m})\end{array}$ & $\begin{array}{c}\text { Microring- } \\
\text { waveguide } \\
\text { Gap (nm) }\end{array}$ & $\begin{array}{c}\text { Launched } \\
\text { Polarization }\end{array}$ & $\begin{array}{c}\text { FSR } \\
(\mathrm{nm})\end{array}$ & $\begin{array}{c}\text { FWHM } \\
(\mathrm{nm})\end{array}$ \\
\hline $\begin{array}{c}\text { This } \\
\text { work }\end{array}$ & 250 & 450 & 5 & 200 & TE & 18.4 & $0.1-0.2$ \\
\hline$[80]$ & 250 & 500 & 5 & 300 & TE & 16 & 0.11 \\
\hline$[78]$ & 250 & 450 & 5 & 250 & TE & 18.1 & 0.11 \\
\hline 79$]$ & 200 & 400 & 5 & 350 & TE & 17.9 & 0.12 \\
\hline
\end{tabular}

\subsection{Wavelength Tuning by Controlling Substrate Temperature}

As discussed previously, tuning of the filter pass-band wavelength was done by changing the resonance wavelength of the microring resonator. The resonance wavelength tuning is realized by thermal tuning the refractive index of the waveguide material, making use of silicon's large thermooptic coefficient. To test the thermal tuning property of the silicon-based microring resonator, drop port transmitted spectra of the same microring resonator were measured at different equilibrium temperatures (from $12^{\circ} \mathrm{C}$ to $60^{\circ} \mathrm{C}$ ) to observe peak shift. The temperature of the chip was controlled by a peltier thermoelectric module and monitored by a thermocouple attached on the sample surface in this experiment. Figure 5.6 shows the normalized transmitted power spectra taken at different sample temperature with TE input polarization. All curves were normalized to their maxima and only a segment of the spectrum from 1570 to $1580 \mathrm{~nm}$ 
was shown in the figure to demonstrate the shifts of a resonance peak with TE input polarization.

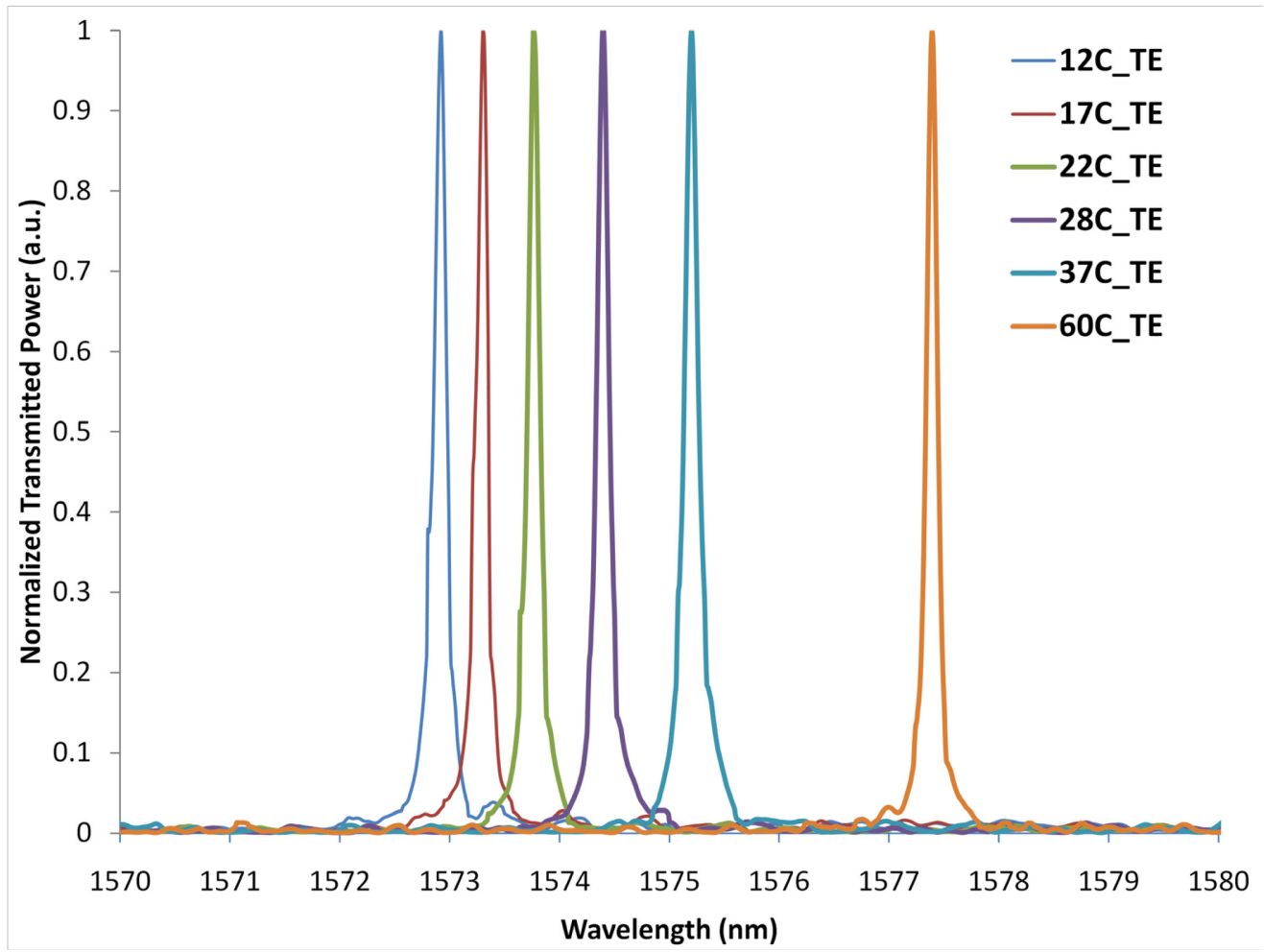

Figure 5.6 Normalized drop port transmitted power spectra taken at different sample temperature with TE input polarization. Legends shows the sample temperature when spectrum was measured.

From the spectra, we can observe that the resonance peak shifts red when the sample is heated up and shifts blue when cooling. The resonance shift compared with the resonance at room temperature $\left(22^{\circ} \mathrm{C}\right)$ versus temperature change for all five $\mathrm{TE}$ resonance peaks within the laser tunable range was plotted in Figure 5.7. The shift of different resonance peaks was found to be the same when temperature change is the same. A clear linear relation was observed between the resonance shift and temperature change with a slope of $0.095 \mathrm{~nm} /{ }^{\circ} \mathrm{C}$. Similar wavelength tuning coefficient was reported previously with similar silicon-based microring resonators [43, 81]. For TM resonances, linear resonance shifts were also observed versus temperature changes. The slope was 
determined to be $0.076 \mathrm{~nm} /{ }^{\circ} \mathrm{C}$. The measured thermooptic tunability for the TE and TM mode were found to be slightly larger compared to the simulated ones of $0.075 \mathrm{~nm} /{ }^{\circ} \mathrm{C}$ and $0.063 \mathrm{~nm} /{ }^{\circ} \mathrm{C}$. The reason for the larger value is still not clear. A possible explanation could be the bending effect or the effect due to the deposited $\mathrm{Si}_{3} \mathrm{~N}_{4}$ layer, which were not considered in the simulation.

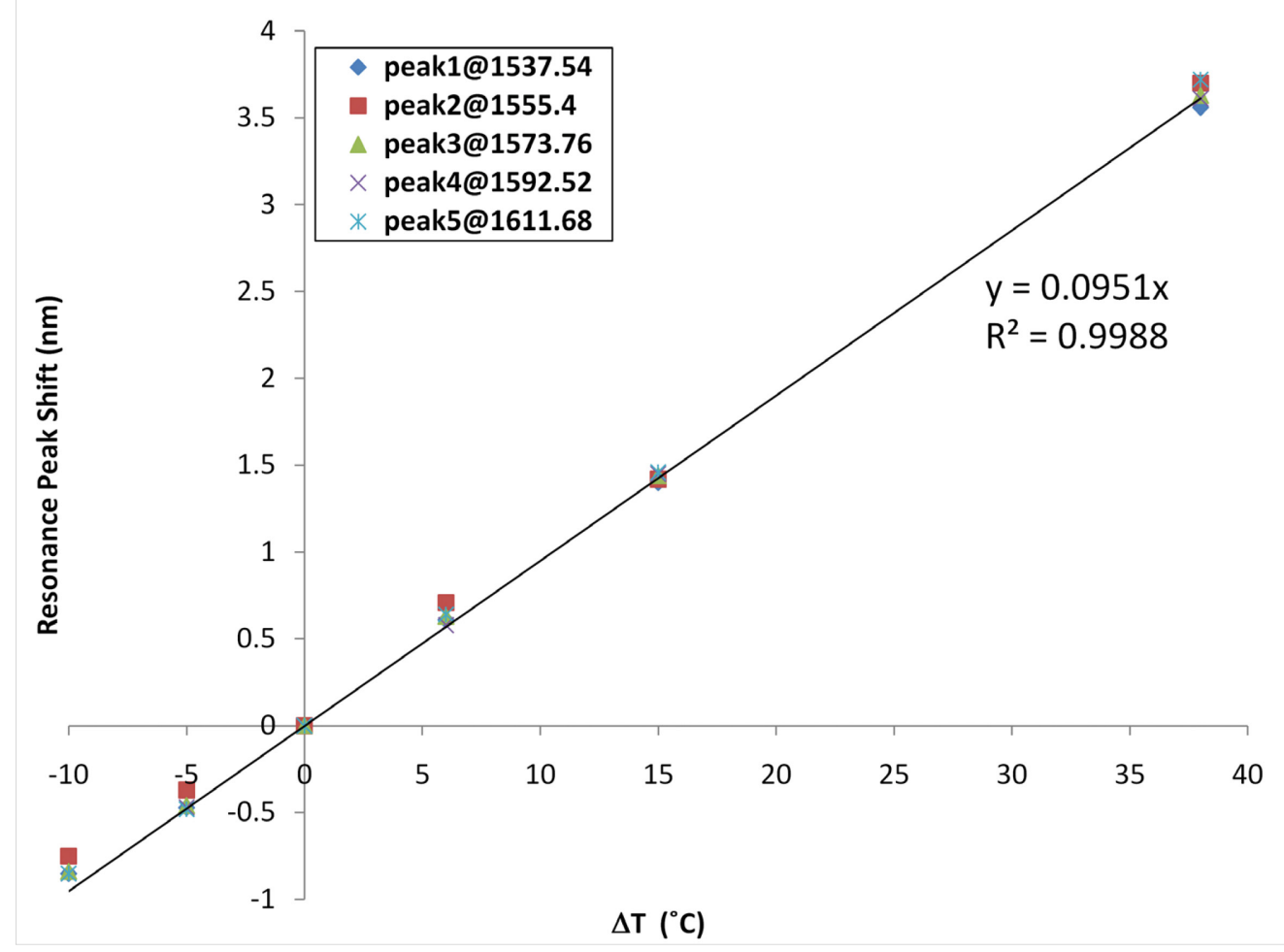

Figure 5.7 Measured resonance shift versus temperature change of five TE resonance peaks when tuning the sample temperature.

\subsection{Wavelength Tuning by Micro-heater}

The resonance wavelength tuning of the same microring resonator by microheater on top of it was then tested. Constant current was applied to the heater using a pair of electric probes. While keeping the bottom of the sample at room temperature, the 
spectra was taken a minute after the current was applied to make sure the device reached thermal equilibrium. As the microing resonator only got heated up with current applied, only red shift was observed. The TE resonance spectra with various current applied to the heater is shown in Figure 5.8.

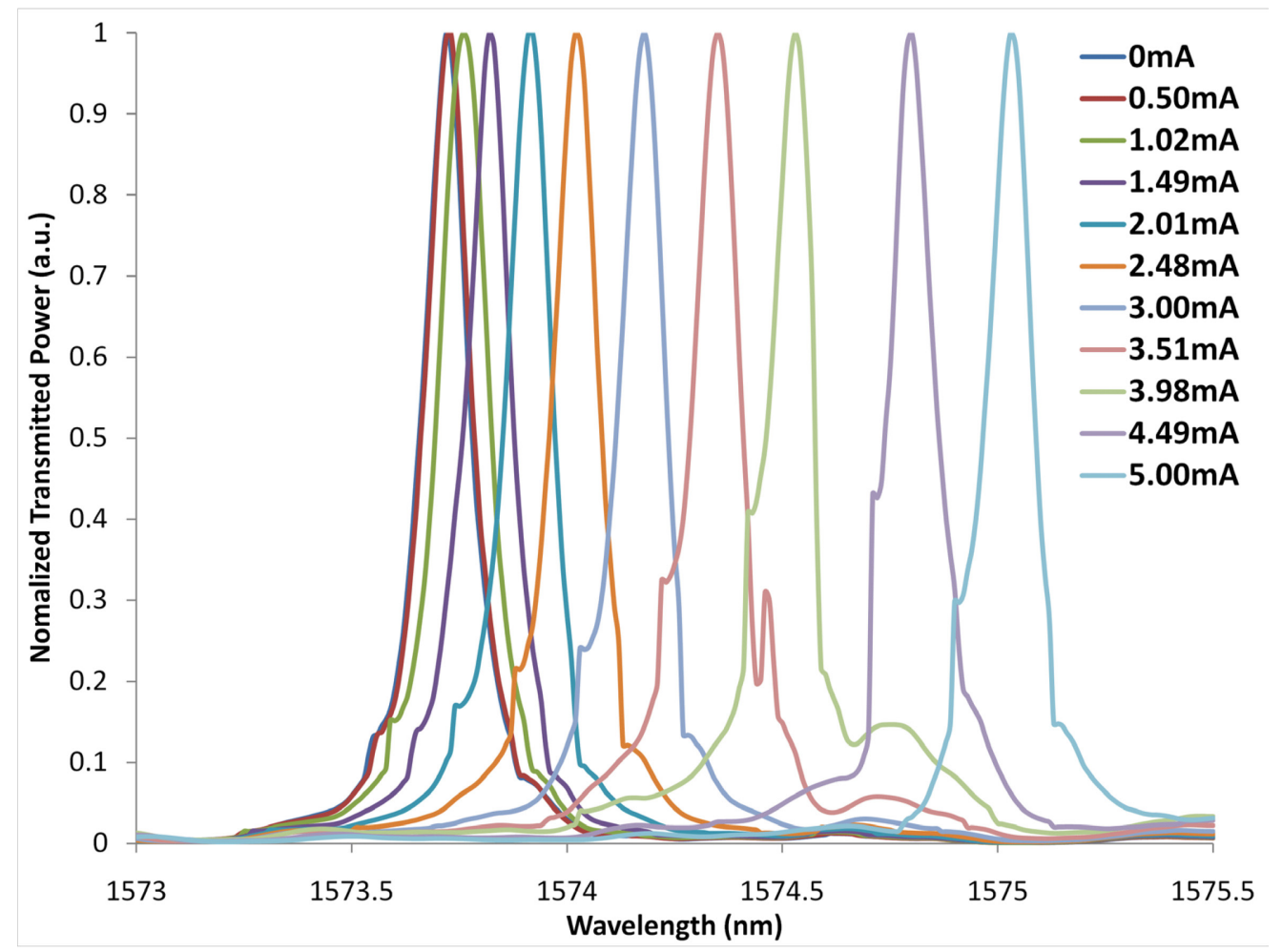

Figure 5.8 Normalized drop port TE transmitted power spectra taken at various heating currents. Legend shows the constant currents applied to the micro-heater when the spectrum was measured.

The resonance shift versus the micro-heater current is plotted in Figure 5.9(a). As expected, the resonance shift was proportional to the power applied to the micro-heater, i.e., the square of micro-heater current as demonstrated in Figure 5.9(b), assuming the resistance change of the micro-heater over temperature is negligible. The slope was determined to be $0.0522 \mathrm{~nm} / \mathrm{mA}^{2}$. Using the simulated micro-heater resistance of $620 \Omega$ and the thermooptic tunability of $0.095 \mathrm{~nm} /{ }^{\circ} \mathrm{C}$, the heating coefficient was calculated to be $1.13 \mathrm{~mW} /{ }^{\circ} \mathrm{C}$, about $40 \%$ higher when compared to the simulated one of $0.81 \mathrm{~mW} /{ }^{\circ} \mathrm{C}$. It is 
possibly because of imperfect heat sink or the larger convective heat transfer caused by the air flow in the environment.
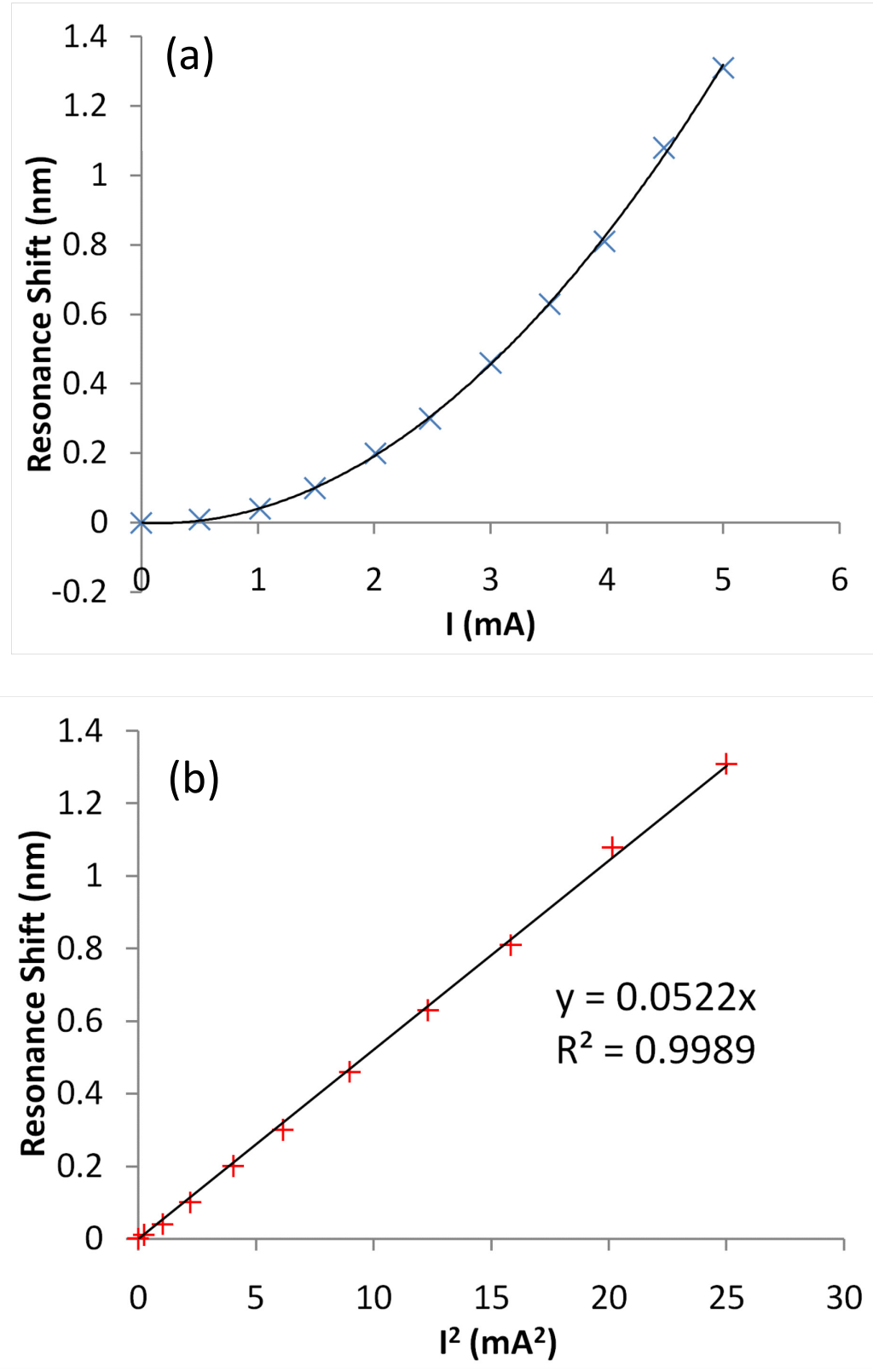

Figure 5.9 Resonance shift versus (a) micro-heater current applied (b) current square. 


\subsection{Time Response of Micro-heater Tuning}

Time response is a key measurement of the performance of a tunable filter. To characterize the time response of the filter, we study the time response of the following typical switching process: A signal with wavelength $\lambda_{t}$ (target resonance wavelength for the filter) is transmitted from the input port of the filter while the filter is in "idle" state with a resonance at $\lambda_{0}$, and with no current applied to the heater. Starting at the moment $\mathrm{t}=0$, a constant current is applied to the heater. The temperature of the microring device increases until it reaches the thermal equilibrium; while the resonance wavelength increases along with the temperature and finally tunes to and stays at $\lambda_{\text {t. }}$ With the resonance tuned to $\lambda_{t}$, the input signal was switched from the through port to the drop port. The thermal switching of the microring filter between $\lambda_{0}$ and $\lambda_{t}$ is characterized by four time constants, with each time constant corresponding to a different tuning or detuning process. During heating cycle, we observe detuning from $\lambda_{0}$, and tuning to $\lambda_{t}$; while during the cooling cycle, the detuning from $\lambda_{\mathrm{t}}$, and tuning to $\lambda_{0}$.

To test the switching time constants, an experiment was setup as described below. While keeping the backside of the chip always at room temperature, the tunable laser wavelength was first set to a predefined $\lambda_{t}$, which is larger than $\lambda_{0}$. The micro-heater was connected to a HP8116A function generator, which is capable of generating waveforms with frequency up to $50 \mathrm{MHz}$ and voltage up to $16 \mathrm{~V}$ peak to peak. The voltage amplitude of the square wave was set to the value that tunes the resonance to $\lambda_{\mathrm{t}}$. A $100 \mathrm{~Hz}$ square wave voltage with $10 \%$ "on" duty cycle was applied to the micro-heater. The small "on" duty cycle time was set to avoid heat building up with high power heating. The period 
was significantly longer than the switching time, which allows equilibrium to be reached during both "heater on" periods and "heater off" (cooling) periods. A Newport 818-IR photodetector was used at the output. The detector has a rising time of $\sim 1 \mu \mathrm{s}$. The Newport $1835 \mathrm{C}$ optical meter connected to the photodetector can output a voltage waveform proportional to the detected power. The voltage waveform and the voltage applied to the heater were simultaneously monitored by an HP 54645D oscilloscope which is able to detect voltage variation up to $400 \mathrm{MHz}$. Using the oscilloscope, the power transients of tuning process to $\lambda_{t}$ and the detuning process from $\lambda_{t}$ were detected at the rising and falling edges of the heater voltage and recorded. By setting the laser wavelength to $\lambda_{0}$ while keeping the driving voltage of the micro-heater, the detuning process from $\lambda_{0}$ and tuning process to $\lambda_{0}$ were detected in the same manner.

Measurements were taken tuning the resonance peak of $\lambda_{0}=1573.76 \mathrm{~nm}$ and setting $\Delta \lambda=\lambda_{\mathrm{t}}-\lambda_{0}$ to multiples of $0.2 \mathrm{~nm}$, namely $0.2,0.4,0.8,1.6,3.2$ and $6.4 \mathrm{~nm}$. Figure 5.10 (a)-(d) shows the normalized output power for the four different processes. For all curves, the heater driving voltages turns on or off at $\mathrm{t}=0$. Note that the plots showing the tuning processes have a much longer time scale compare to the detuning one. That is because the tuning process is usually much slower as compared to the detuning process. 

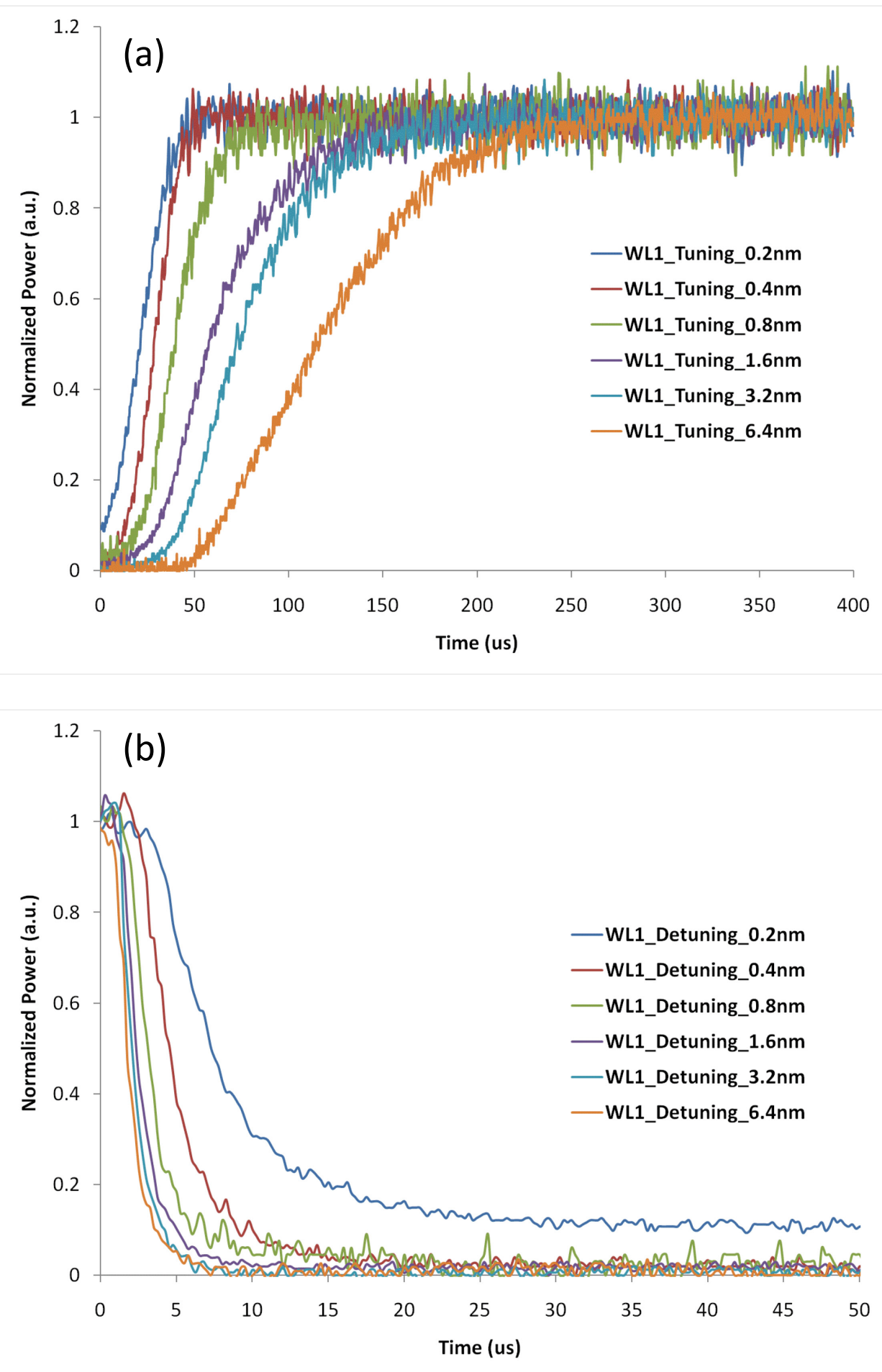

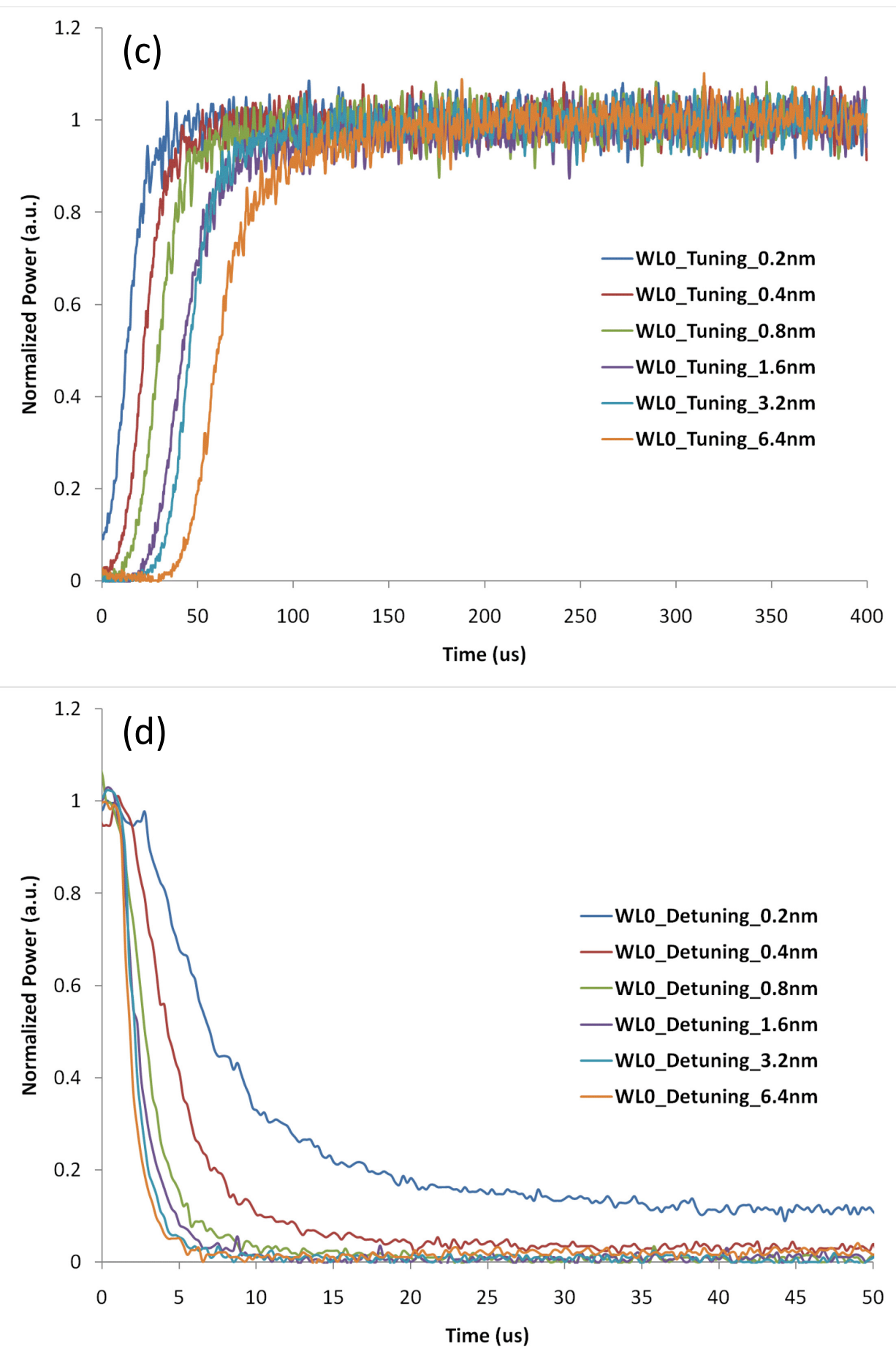

Figure 5.10 Measured output power transients for processes of (a) tuning to $\lambda_{t}(b)$ detuning from $\lambda_{t}$ (c) tuning to $\lambda_{0}(\mathrm{~d})$ detuning from $\lambda_{0}$, when tuning the resonance with wavelength $\lambda_{0}=1573.76 \mathrm{~nm}$. Different curves in each plot shows the measurements with different wavelength shift $\left(\Delta \lambda=\lambda_{t}-\lambda_{0}\right)$ of $0.2,0.4,0.8$, 1.6, 3.2 and $6.4 \mathrm{~nm}$ Each curve was normalized related to its maximum. 
The tuning time response for the same resonance at $1573.76 \mathrm{~nm}$ was simulated using the time response of the average microring temperature change modeled in Chapter III. Given the resonance wavelengths at the beginning and the end of the heating and cooling process, the simulated resonance wavelength time responses during the processes were calculated. Using a Lorentzian lineshape for the resonance peak fitted with the measured FWHM of $0.13 \mathrm{~nm}$ for the $1573.76 \mathrm{~nm}$ peak, the simulated tuning time responses for the four processes as measured and demonstrated in Figure 5.10 were calculated and shown in Figure 5.11. By comparing the measured responses with simulated responses, we can find close agreement between them. Measurements are taken and simulations are repeated for two other peaks to gather more data. Figure 5.12 summarized the measured and simulated time response by plotting the rise/fall delay time as a function of $\Delta \lambda$ for three resonance peaks at $1573.76 \mathrm{~nm}, 1592.52 \mathrm{~nm}$ and $1611.68 \mathrm{~nm}$ at room temperature. The rise/fall delay time $\left(\tau_{\mathrm{dr}} / \tau_{\mathrm{df}}\right)$ for the tuning/detuning process is defined as the time between the heating power on/off time and the photodetector voltage signal rising/falling to $50 \%$ of the maximum power. Measured FWHM of the resonance peaks was used for calculation of the rise/fall delay time responses. The peaks with FWHM measurement are shown in the insets of Figure 5.12. 

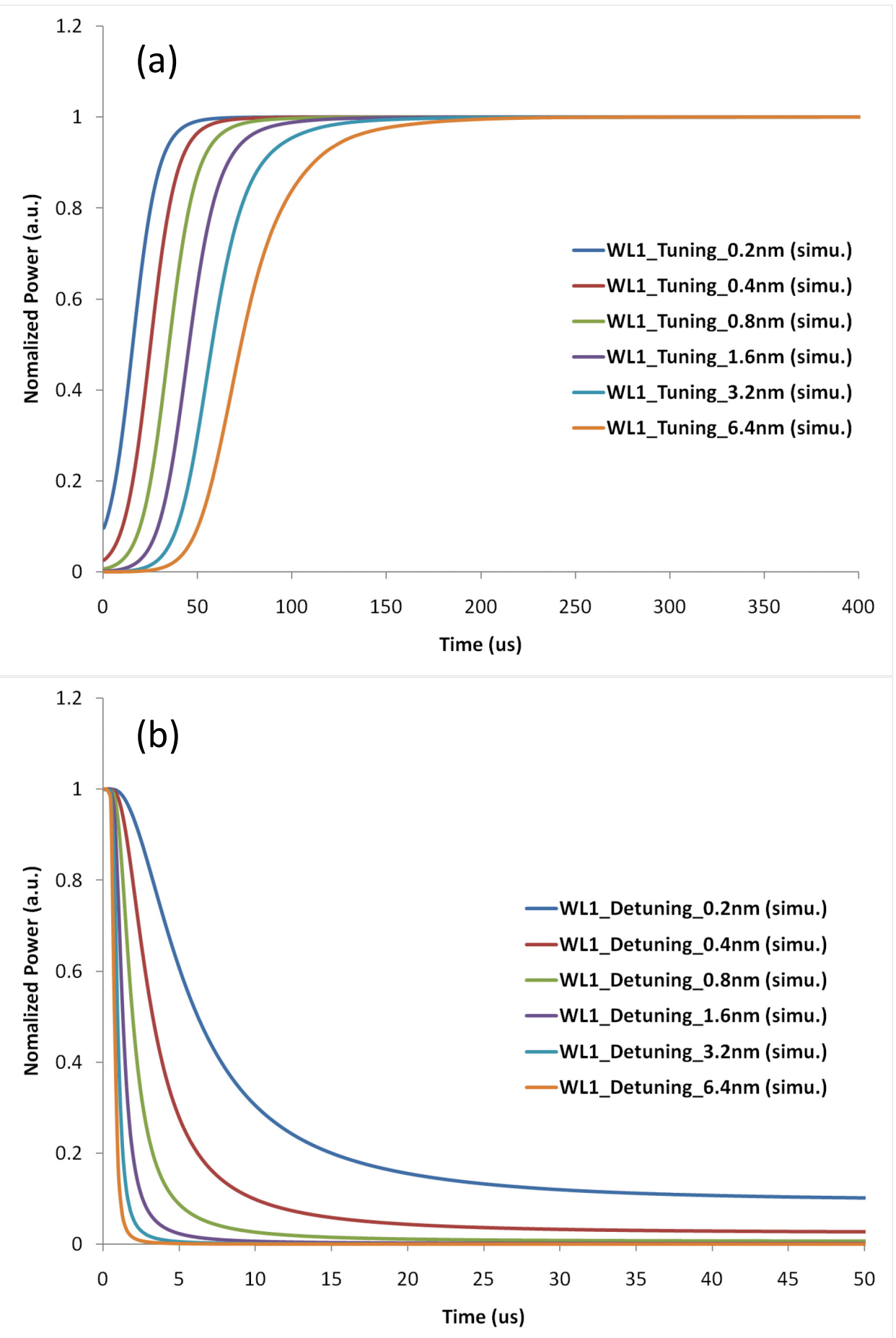

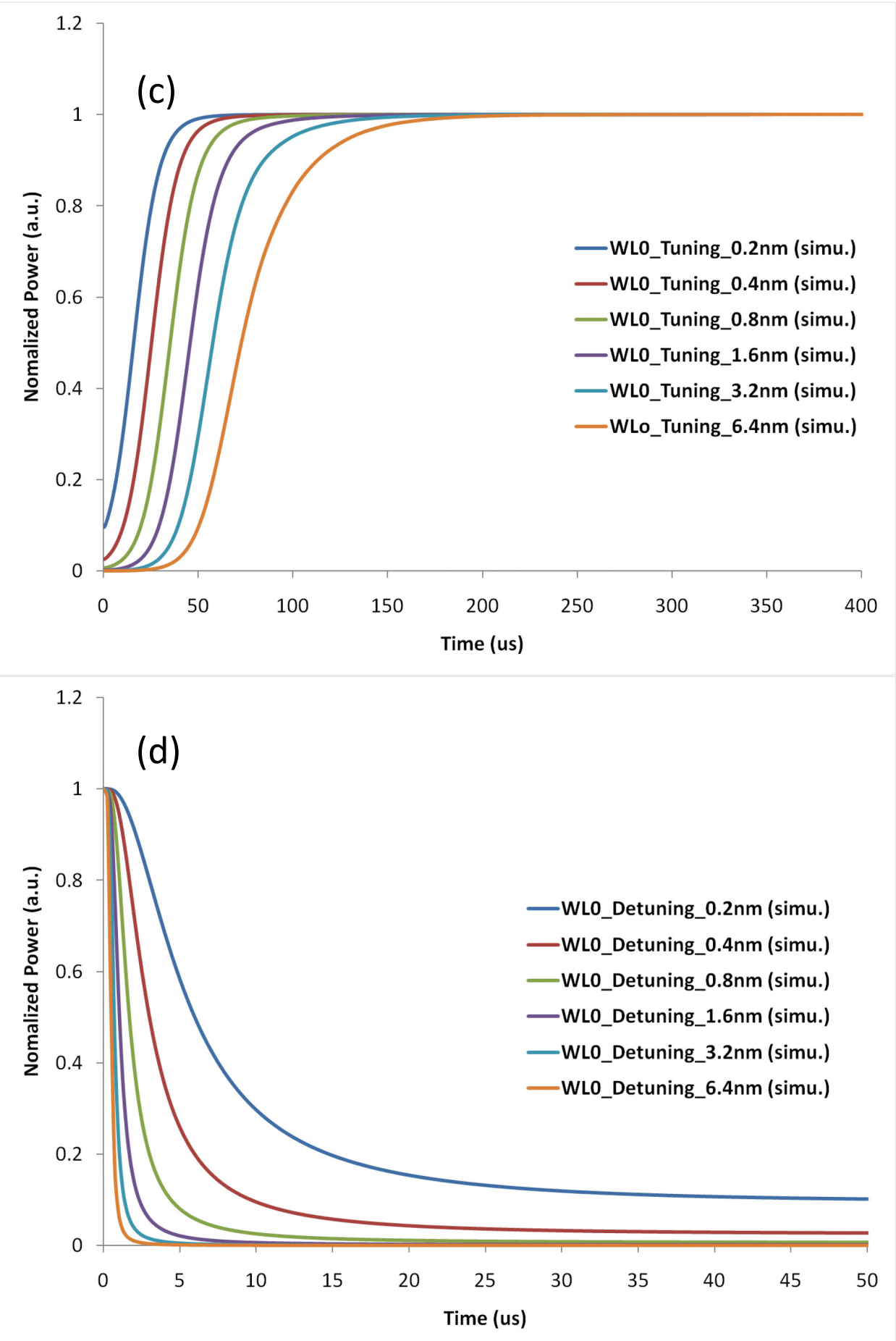

Figure 5.11 Simulated output power transients for processes of (a) tuning to $\lambda_{t}(b)$ detuning from $\lambda_{t}$ (c) tuning to $\lambda_{0}(\mathrm{~d})$ detuning from $\lambda_{0}$, when tuning the resonance with wavelength $\lambda_{0}=1573.76 \mathrm{~nm}$. Different curves in each plot shows the measurements with different wavelength shift $\left(\Delta \lambda=\lambda_{t}-\lambda_{0}\right)$ of $0.2,0.4,0.8$, 1.6, 3.2 and $6.4 \mathrm{~nm}$ Each curve was normalized related to its maximum. 


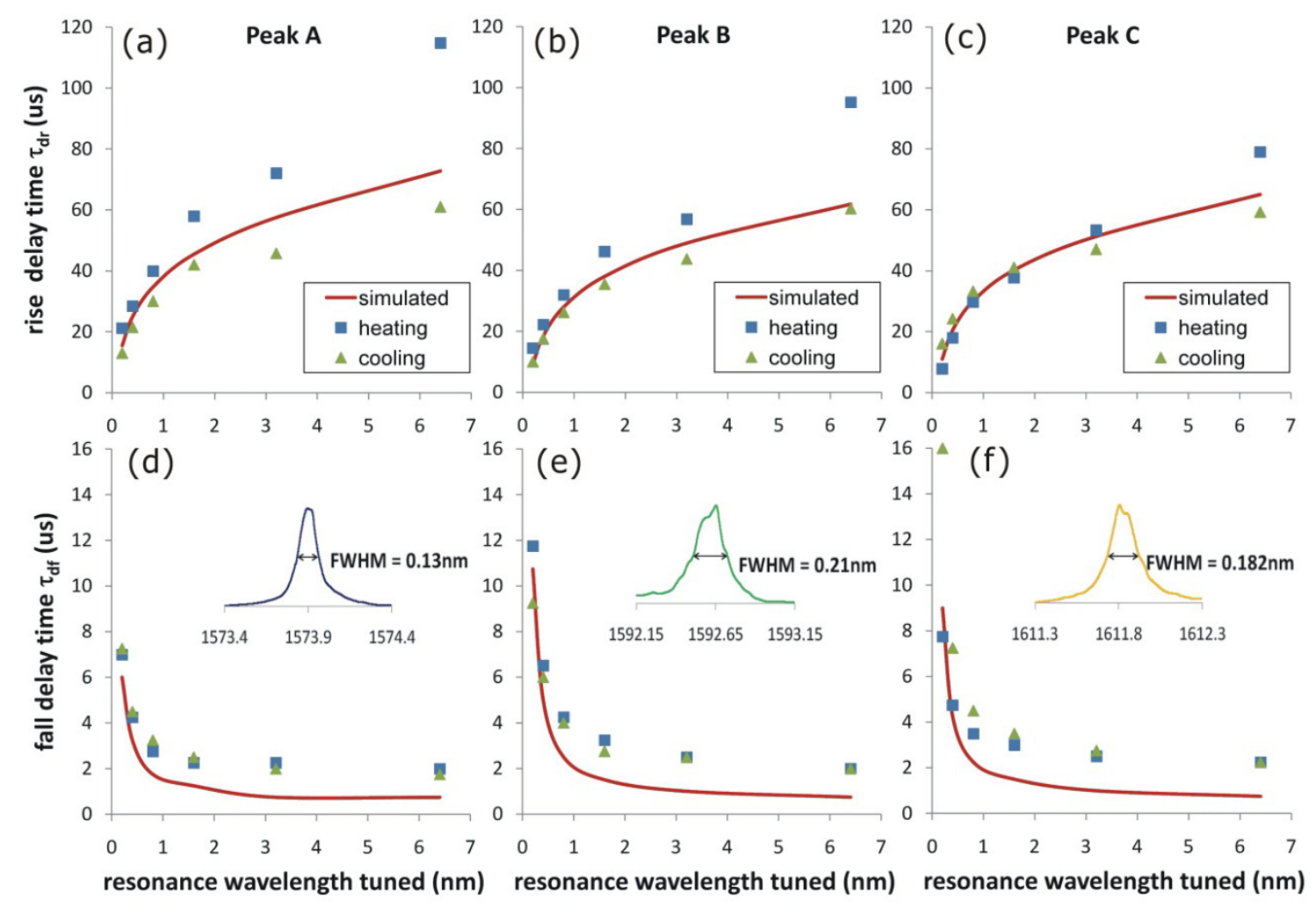

Figure 5.12 Plots of tuning rise delay times of room-temperature resonance peaks at (a) $1573.76 \mathrm{~nm}$ (Peak A), (b) $1592.65 \mathrm{~nm}$ (Peak B) and (c) $1611.80 \mathrm{~nm}$ (Peak C) as well as plots of fall delay times of the peaks (d), (e) and (f), respectively. The curves are simulated results and the dots are measured values. The insets are the corresponding peak spectra. Note the different time scale between rise and fall plots.

As shown in Figure 5.12, the rise/fall times are in close agreement with the simulated values. For different resonance peaks, the simulated switching times are different due to the slightly different FWHM, in spite of the same temperature time response. The fall times (the detuning process) are typically much shorter than the rise (tuning) times, due to the initial rapid increase of the temperature as seen in Figure 3.13(a). On the other hand, the quasi-exponential nature of the temperature tuning to the final value, either through cooling or heating, leads to a slow natural log trend for higher resonance shifts. The cooling and heating rise-delay times are not identical due to the asymmetry of the measured lineshape, as seen in the insets of Figure 5.10. Furthermore, a 
stronger deviation from the simulated values can be seen in the high resonance shift region, when tuning by heating as seen in Figure 5.12(a-c). Parasitic feedline heating, imperfect heat sinking, convection or perhaps strain-induced anisotropy effects could also be the cause, and deserve further exploration. The measured fall times are in agreement with the simulation, being higher than the simulation due to the $\sim 1 \mu$ s photodetector rise/fall time.

Silicon based thermooptic switches usually have $\mu$ s or sub- $\mu$ s response and $\mathrm{mW}$ power consumption. Comparing the performance of different switches is difficult because 1) they are based on different structures 2) the speed and power consumption depend on the distance between the heat source and light circuit. Table 5.4 attempts to summarize the time response and power consumption of different thermooptic switches based on silicon. The Mach-Zehnder interferometer is a popular platform for thermooptic switch $[35,82,84]$ due to its high speed. The microring resonator $[37,85]$ and Fabry-Perot cavity [83] can be used as wavelength-selective switches which add an important function. Our device is slower compared to the other devices listed, due to the far distance between heater and optical layer and the need for wide wavelength tuning range. The power consumption for our device is relatively low for switching applications with no need for large wavelength tuning. With the ring-shape heater, an improvement in performance is expected. 


\subsection{Summary}

In this chapter, we experimentally characterized the thermally wavelength tunable microring resonator based filter. The compact $10 \mu \mathrm{m}$-diameter microring resonator has a $\mathrm{Q}$ of around 10,000. The wide tunable range $(>6.4 \mathrm{~nm})$ and large free spectrum range $(\sim 18 \mathrm{~nm})$ of the switch element enables wavelength reconfigurable multi-channel matrix switching by wavelength multiplexing and demultiplexing. Average rise delay time of $14 \mu$ s with low switching power of $3.15 \mathrm{~mW}$ has been achieved with $0.2 \mathrm{~nm}$ wavelength tuning and $78 \mu \mathrm{s}, 104 \mathrm{~mW}$ for $6.4 \mathrm{~nm}$ tuning. Fall delay times are usually less than $10 \mu \mathrm{s}$. Thermal simulations show agreement within $15 \%$ error with the measurements up to $3.2 \mathrm{~nm}$ tuning. The compact size of the switch shows its potential as an active element in photonic integrated circuits.

Table 5.4 Comparison of performances of silicon-based thermooptic switch

\begin{tabular}{|c|c|c|c|c|}
\hline Reference & Platform & $\begin{array}{l}\text { Rise Time } \\
(\mu \mathrm{s})\end{array}$ & $\begin{array}{l}\text { Power } \\
(\mathrm{mW})\end{array}$ & Note \\
\hline This work & $\begin{array}{l}\text { Microring } \\
\text { Resonator }\end{array}$ & $\begin{array}{l}14 \\
78\end{array}$ & $\begin{array}{l}3.15 \\
104\end{array}$ & $\begin{array}{c}0.2 \mathrm{~nm} \text { tuning } \\
6.4 \mathrm{~nm} \text { tuning } \\
1.4 \mu \mathrm{m} \text { from heater }\end{array}$ \\
\hline$[85]$ & $\begin{array}{l}\text { Microring } \\
\text { Resonator }\end{array}$ & 5 & $\begin{array}{l}17 \\
57\end{array}$ & $\begin{array}{c}0.05 \mathrm{~nm} \text { tuning } \\
0.25 \mathrm{~nm} \text { tuning } \\
0.3 \mu \mathrm{m} \text { from heater }\end{array}$ \\
\hline$[35]$ & Mach-Zehnder & $<3.5$ & 50 & $1 \mu \mathrm{m}$ from heater \\
\hline$[84]$ & Mach-Zehnder & $<1$ & 250 & $1 \mu \mathrm{m}$ from heater \\
\hline$[82]$ & Mach-Zehnder & 0.6 & $<0.1$ & Self-heating, multi-stage \\
\hline$[83]$ & Fabry-Perot & 1.9 & 10 & $1 \mu \mathrm{m}$ from heater \\
\hline
\end{tabular}




\section{APPLICATIONS}

The wavelength-tunable filter was designed as ROADM for DWDM optical communication systems. In this chapter, two applications are demonstrated towards this objective with the designed wavelength-tunable filter: 1) an on-chip $1 \times 4$ and $4 \times 4$ switch and 2) on-chip wavelength control of fiber laser.

The former work was to demonstrate high port count DWDM system with scalable ROADM structures using the wavelength-tunable filter. It was a collaboration work with New Span Opto-technology, Inc and the Photonics and Microdevice Lab in the University of Miami. In this work, the $1 \mathrm{x} 4$ and $4 \mathrm{x} 4$ switch samples were designed and fabricated by the author. And the characterization was performed by our partners.

The later work was towards the integration of lasers on silicon photonic chips. The realization of this application helps to evaluate the feasibility of reconfigurable multiwavelength laser generation by the wavelength-tunable filter. Reconfigurable multiwavelength lasera are also a subject of interest for DWDM optical communication system.

\subsection{1x4 and 4x4 Reconfigurable Photonic Switch}

Many wavelength channels can be selected through local thermal tuning of resonators for reconfigurable switching and wavelength-division multiplexing and demultiplexing. The tunable filter investigated previously in this work has an FSR of $18 \mathrm{~nm}$ and FWHM in the range 0.1-0.2 nm. Given the narrow linewidths of the microring resonators, $0.2 \mathrm{~nm}$ or $25-\mathrm{GHz}$ channel spacing is appropriate for our switch device. Also, a wide-range wavelength tuning of at least $6.4 \mathrm{~nm}$ was demonstrated which enables 32 
side by side channels within the tunable range. With the compact size of the filter $(50 \mu \mathrm{m}$ $\mathrm{x} 50 \mu \mathrm{m}), 1 \mathrm{xN}$ or $\mathrm{NxN}$ reconfigurable photonic switch can be realized on a millimeter size chip by integrating multiple filters.

To demonstrate the idea of $1 \mathrm{xN}$ and $\mathrm{NxN}$ reconfigurable photonic switch, we designed, fabricated and configured a $1 \times 4$ [47] and a $4 \times 4$ [48] switch. The switch was designed by using multiple identical input and output microrings on a common bus waveguide, with a micro-heater located on each of the rings. The layout diagram of the $1 \times 4$ and $4 \times 4$ reconfigurable photonic switch was shown in Figure 6.1(a) and (b). For the $1 \times 4$ switch, a multiplexed signal which combines the four input channel signals is sent to the input waveguide. These four signals are then selectively routed to the corresponding output ports by tuning the resonances of the microring resonators to the corresponding channels. And for the $4 \mathrm{x} 4$ configuration, the four input signals are sent to the four input waveguides. The input microring resonators multiplexed the four input channel signals to the bus waveguide. A routing path from input end to output end is formed when an input microring and an output microring are tuned to a matched resonance wavelength. For $4 \times 4$ configuration, we designate input and output sides for easier reference. The device can operate bi-directionally. 
(a)
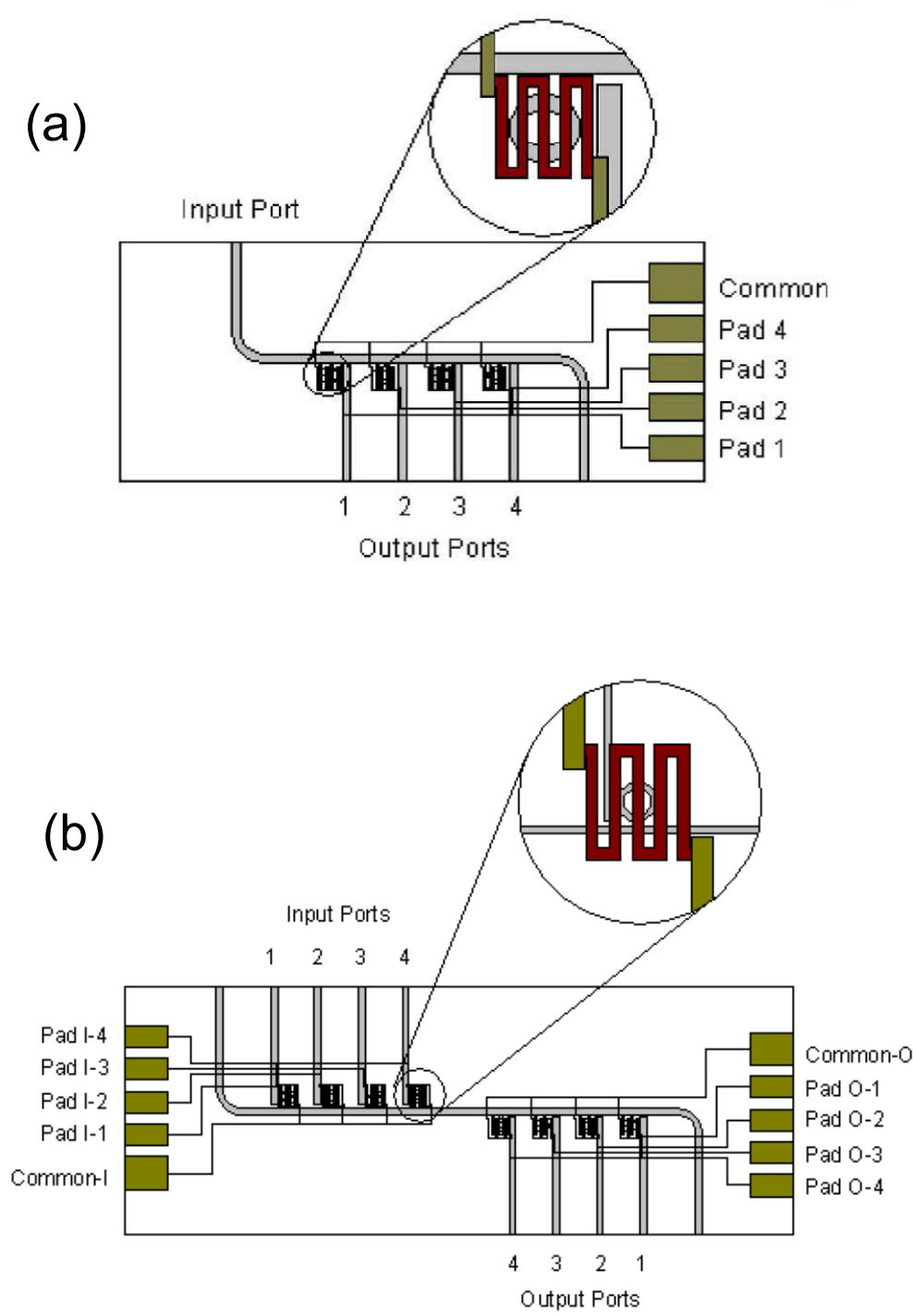

Figure 6.1 Layout diagrams of the (a) $1 \times 4$ and (b) $4 \times 4$ wavelength reconfigurable photonic switches based on thermally tunable microring resonators on SOI substrate.

The fabrication process for the $1 \times 4$ and $4 \times 4$ reconfigurable switch is identical to the single thermally tunable filter/switch as described previously in this work. Only the designs of them are different. The setup used to characterize the devices are slightly different to the one described in Chapter V, the details of the setup can be found in reference [47]. To implement local thermooptic tuning with micro-heaters, a printed circuit board (PCB) was fabricated with electric wires bonded to the electrode pads on the 
switch device. Thermal silver epoxy was used to bond fine stripped magnet wires with a diameter of $45 \mu \mathrm{m}$ to the PCB and the electrode pads on the device. Figure 6.2 shows a photo of the TEC-controlled $4 \mathrm{x} 4$ switch device and the PCB assembly.

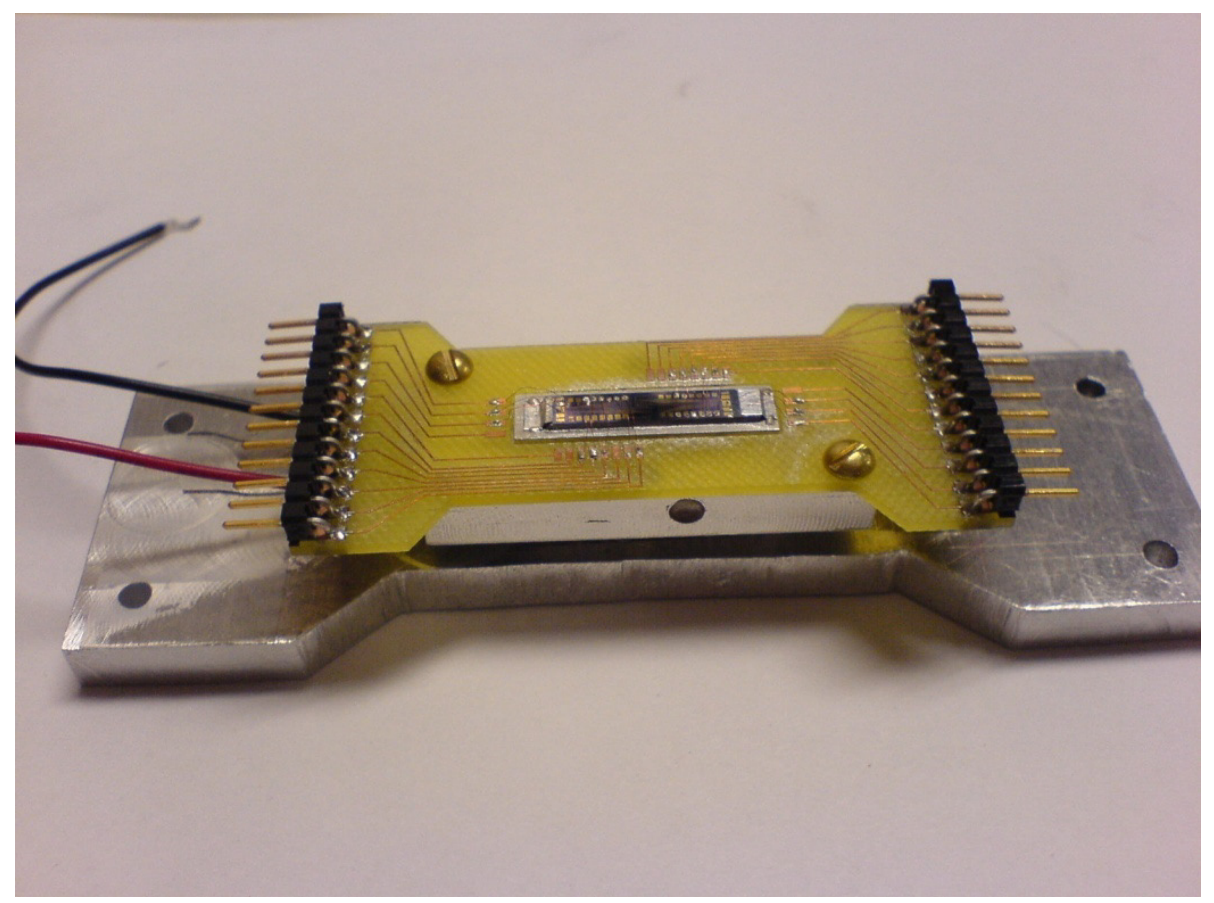

Figure 6.2 Picture of the TEC temperature-regulated 4x 4 switch device and PCB assembly.

For the $1 \times 4$ configuration, four ITU grids $\left(\lambda_{1}, \lambda_{2}, \lambda_{3}\right.$, and $\left.\lambda_{4}\right)$ of $1562.43,1562.83$, 1563.23, and $1563.63 \mathrm{~nm}$ were chosen as the output channels (50-GHz adjacent channel spacing) of the switch device, so that these channels are well separated from each other with minimal crosstalk. For a $1 \times 4$ wavelength reconfigurable switch, there are a total of $4 !=24$ switching combinations, with Figure 6.3 showing one switching state of the device, where input signals $\lambda_{1}, \lambda_{2}, \lambda_{3}$, and $\lambda_{4}$ are routed to output ports $4,3,1$, and 2 , respectively. It was found that a heating current of approximately $5 \mathrm{~mA}$ was needed to tune a resonance across four wavelength channels of $1.2 \mathrm{~nm}$. 


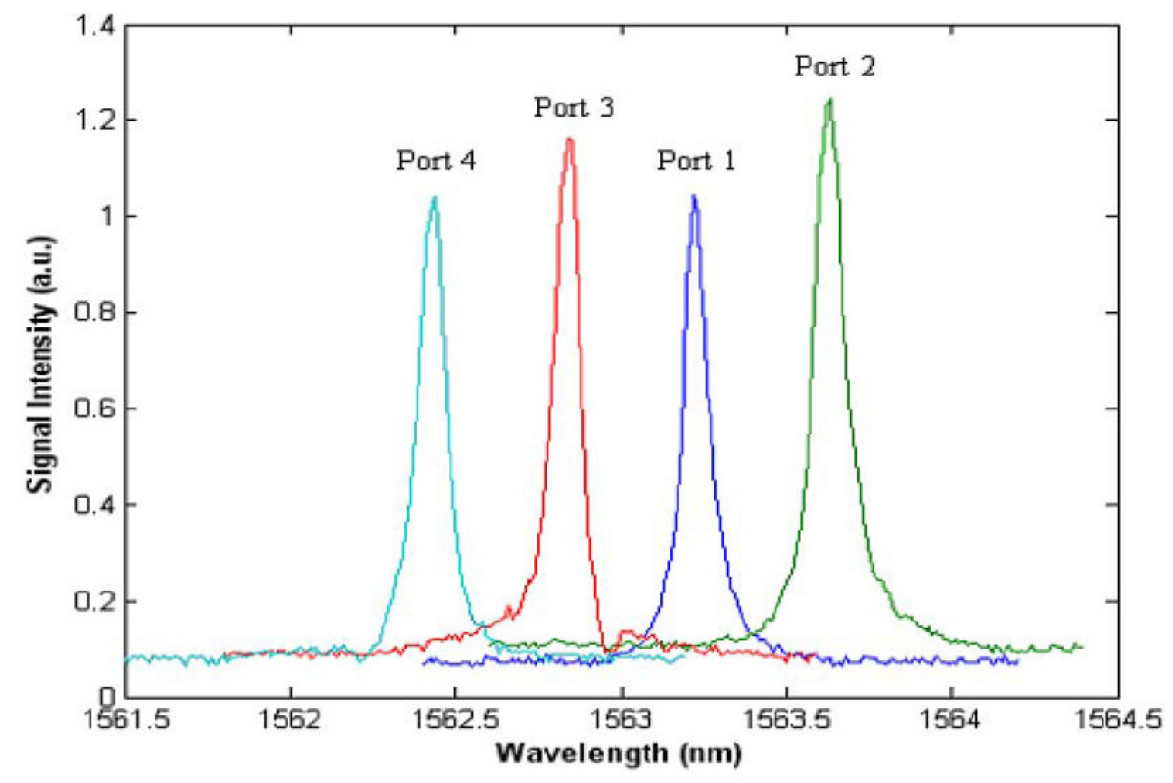

Figure 6.3 Output spectrum of the device showing wavelength routing of $1562.43,1562.83,1563.23$, and $1563.63 \mathrm{~nm}$ to output ports $4,3,1$, and 2 , respectively

For the $4 \times 4$ configuration, the resonant wavelength peaks of the eight microring resonators and the resistances of their corresponding micro-heaters are presented in Table 6.1. For this $4 \times 4$ photonic switch, the following four ITU grids were chosen as the wavelength channels: $\lambda_{1}=1562.23 \mathrm{~nm}, \lambda_{2}=1562.63 \mathrm{~nm}, \lambda_{3}=1563.03 \mathrm{~nm}$ and $\lambda_{4}=$ $1563.43 \mathrm{~nm}$. As a result, there are total $4 ! \times 4 !=576$ switching configurations. Figure 6.4 shows one switching configuration with input 1 and output 4 , input 2 and output 2 , input 3 and output 3 , and input 4 and output 1 microring pairs, tuned to $\lambda_{1}, \lambda_{2}, \lambda_{3}$, and $\lambda_{4}$, respectively. Given the different resonant wavelength peaks (at a fixed temperature) of the microring resonators and the different resistances of the micro-heaters, as listed in Table 1, the electric heating currents needed to tune the microring resonators to the chosen channel wavelengths have to be determined individually. To determine the heating current to tune a microring resonator to $\lambda_{1}$, for instance, the laser source was fixed at $\lambda_{1}$, and then the heating current was gradually increased to the corresponding micro- 
heater until the microring resonator reached resonance at $\lambda_{1}$. Table 6.2 summarizes the heating currents required to tune the microring resonators to the corresponding wavelength channels, with the values in bold determined for the switching configuration described in Figure 6.4.

Table 6.1 Resonant wavelength peaks of the microring resonators and the resistances of their corresponding micro-heaters

\begin{tabular}{|c|c|c|c|c|c|c|c|c|}
\hline & Input 1 & Input 2 & Input 3 & Input 4 & $\begin{array}{c}\text { Output } \\
1\end{array}$ & $\begin{array}{c}\text { Output } \\
2\end{array}$ & $\begin{array}{c}\text { Output } \\
3\end{array}$ & $\begin{array}{c}\text { Output } \\
4\end{array}$ \\
\hline $\begin{array}{c}\text { Resonant } \\
\text { Wavelength } \\
(\mathrm{nm})\end{array}$ & 1561.29 & 1561.51 & 1561.11 & 1561.71 & 1561.99 & 1561.48 & 1561.30 & 1561.60 \\
\hline $\begin{array}{c}\text { Resistance } \\
(\Omega)\end{array}$ & 963 & 1029 & 1045 & 970 & 782 & 607 & 868 & 784 \\
\hline
\end{tabular}

Table 6.2 Heating currents determined to tune the microring resonators to the corresponding wavelength channels with the current set marked in bold for the switching configuration shown in Figure 6.4

\begin{tabular}{|c|c|c|c|c|}
\hline & $\begin{array}{c}\lambda_{1}(1562.23 \\
\mathrm{nm})\end{array}$ & $\begin{array}{c}\lambda_{2}(1562.63 \\
\mathrm{nm})\end{array}$ & $\begin{array}{c}\lambda_{3}(1563.03 \\
\mathrm{nm})\end{array}$ & $\begin{array}{c}\lambda_{4}(1563.43 \\
\mathrm{nm})\end{array}$ \\
\hline Input Port 1 (I-MR 1) & $\mathbf{4 . 4 0} \mathbf{~ m A}$ & $5.21 \mathrm{~mA}$ & $6.00 \mathrm{~mA}$ & $6.59 \mathrm{~mA}$ \\
\hline Input Port 2 (I-MR 2) & $4.14 \mathrm{~mA}$ & $\mathbf{5 . 0 9} \mathbf{~ m A}$ & $5.83 \mathrm{~mA}$ & $6.48 \mathrm{~mA}$ \\
\hline Input Port 3 (I-MR 3) & $4.27 \mathrm{~mA}$ & $5.05 \mathrm{~mA}$ & $\mathbf{5 . 7 4} \mathbf{~ m A}$ & $6.30 \mathrm{~mA}$ \\
\hline Input Port 4 (I-MR 4) & $3.12 \mathrm{~mA}$ & $4.28 \mathrm{~mA}$ & $5.13 \mathrm{~mA}$ & $\mathbf{5 . 8 7} \mathbf{~ m A}$ \\
\hline Output Port 1 (O-MR 1) & $2.25 \mathrm{~mA}$ & $3.73 \mathrm{~mA}$ & $4.71 \mathrm{~mA}$ & $\mathbf{5 . 5 8} \mathbf{~ m A}$ \\
\hline Output Port 2 (O-MR 2) & $3.75 \mathrm{~mA}$ & $\mathbf{4 . 7 2} \mathbf{~ m A}$ & $5.57 \mathrm{~mA}$ & $6.19 \mathrm{~mA}$ \\
\hline Output Port 3 (O-MR 3) & $4.47 \mathrm{~mA}$ & $5.38 \mathrm{~mA}$ & $\mathbf{6 . 1 8} \mathbf{~ m A}$ & $6.83 \mathrm{~mA}$ \\
\hline Output Port 4 (O-MR 4) & $\mathbf{3 . 7 6} \mathbf{~ m A}$ & $4.75 \mathrm{~mA}$ & $5.56 \mathrm{~mA}$ & $6.36 \mathrm{~mA}$ \\
\hline
\end{tabular}




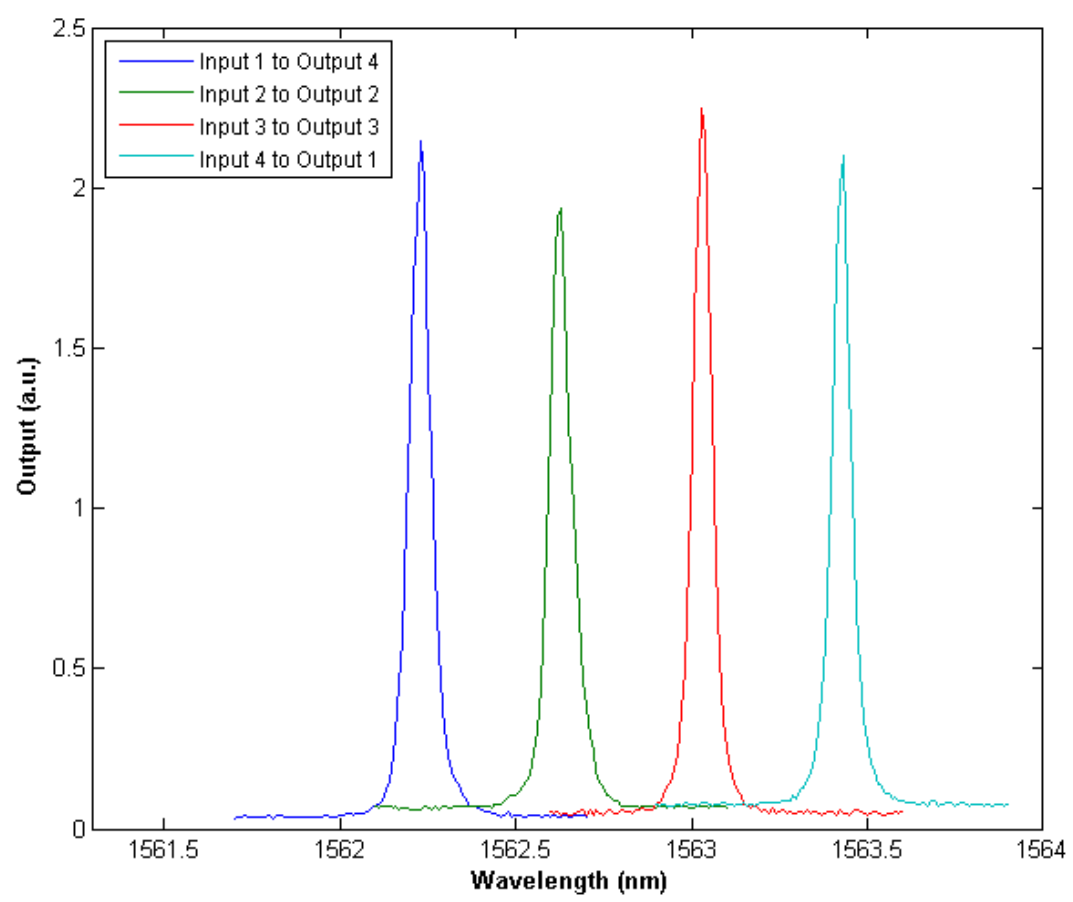

Figure 6.4 One switching configuration of the $4 \times 4$ photonic switch with $\lambda_{1}, \lambda_{2}, \lambda_{3}$, and $\lambda_{4}$ routed from input ports $(1,2,3$, and 4$)$ to output ports $(4,2,3$ and 1$)$, respectively.

Thermal cross-talk between adjacent devices was considered at the design stage. To facilitate standard fiber array connection, adjacent waveguide ports on both input and output sides are spaced at $250 \mu \mathrm{m}$. Simulation using the model described in Chapter III was performed to estimate the temperature change of a microring device due to heating of a heater $250 \mu \mathrm{m}$ away with result shown in Figure 3.13(b). Minimal crosstalk was predicted from the simulation.

The tuning thermal cross-talk between adjacent micro-heaters was evaluated experimentally also. We monitored the peak wavelength shift of one microring while heating up the micro-heater of an adjacent one. There was no peak wavelength shift observed even when a high heating current of $7 \mathrm{~mA}$ was provided to the adjacent microheater, as seen in Figure 6.5. It should be noted that $7 \mathrm{~mA}$ heating current is sufficient to 
tune a microring by approximately $2.5 \mathrm{~nm}$ if supplied directly to the corresponding micro-heater. Therefore, the fabricated $1 \times 4$ and $4 \times 4$ photonic switch has no observable thermal cross-talk.

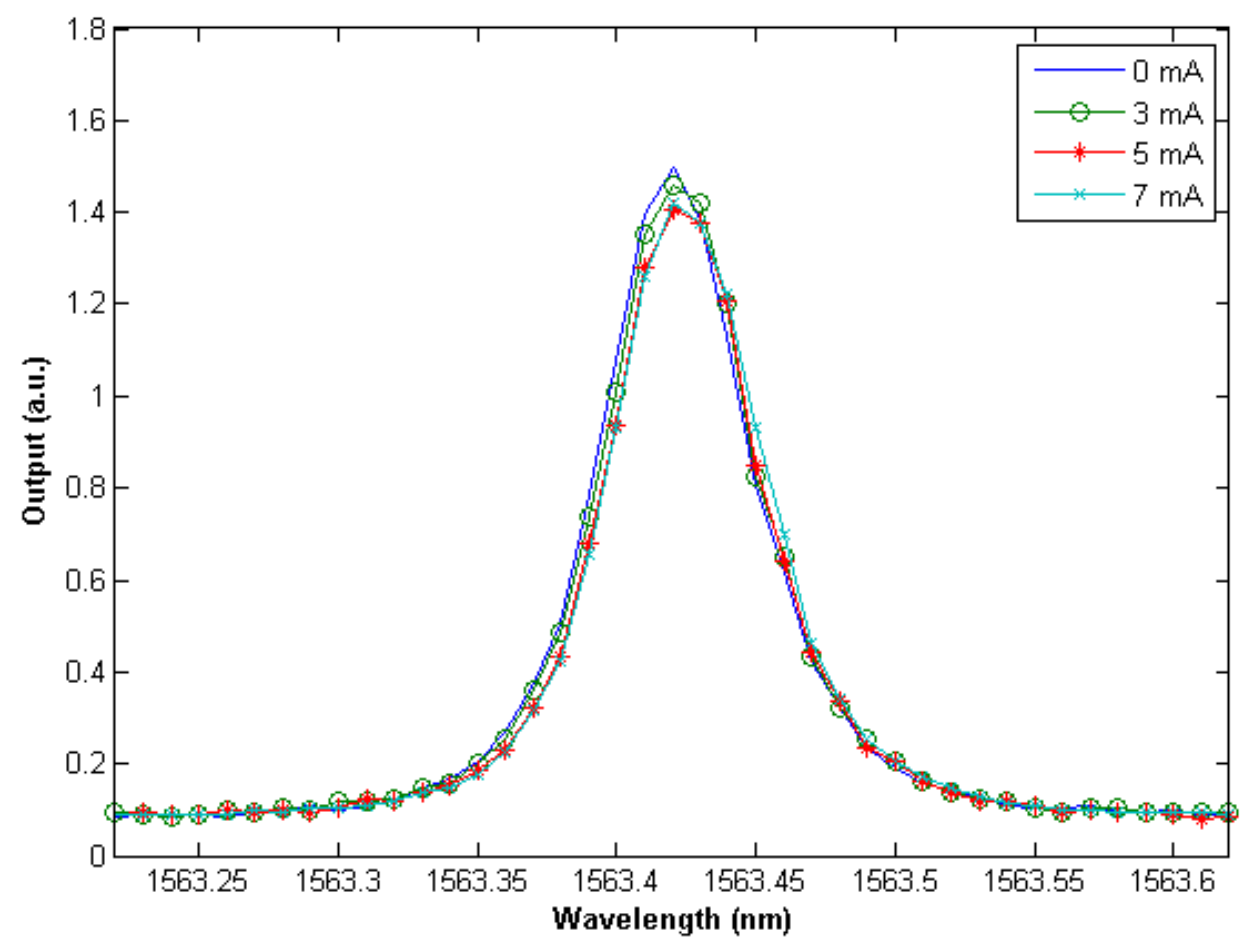

Figure 6.5 Transmission spectrum of a microring resonator with various heating currents sent to an adjacent micro-heater.

In summary, $1 \times 4$ and $4 \times 4$ reconfigurable photonic switches were successfully demonstrated based on the thermally tunable microring resonator with FWHM of 0.1$0.2 \mathrm{~nm}$, tunable range larger than $6.4 \mathrm{~nm}$ and time response in the order of $10 \mu \mathrm{s}$. Independent thermooptic tuning of microring resonators was achieved with highly localized micro-heaters fabricated on the same substrate, yielding no cross-talk between adjacent microring resonators. The results suggest the feasibility of high port-count 
photonic circuitry with dynamic wavelength reconfiguration capability for the development of large-scale integrated photonics.

\subsection{On-chip Wavelength Control of Optical Fiber Laser}

One of the biggest challenges for silicon integrated photonics is the light generation on-chip. Recently, generation of light on silicon using an external optical pump was demonstrated by taking advantage of the strong Raman emission in silicon [49, 50]. This approach requires tuning of the external optical pump wavelength to control emission wavelength.

Using the thermally tunable filter, an alternative approach that integrates on-chip silicon photonic tuning and control of lasing wavelength was demonstrated using an external Erbium-doped fiber amplifier as a gain source. Repeatable and predictable tuning of the filter wavelength is achieved. The use of an Er+-doped fiber with heterogeneously broadened gain enables the generation of multiple arbitrary wavelengths by a single chip with each wavelength independently tuned. With a small footprint, dozens, if not hundreds of such devices can be integrated on a single Si-photonic chip with a single external EDFA, leading to individual wavelength control of DWDM channels. This work was published in [65]

A schematic of the tunable laser experiment is shown in Figure 6.6. The two major components of the tunable laser are the Erbium-doped fiber (EDF) and the siliconbased tunable filter. A piece of EDF serves as the gain media which is pumped by a $980 \mathrm{~nm}$ laser diode. The amplified light from the EDF is coupled to the input of the 
silicon-based intracavity wavelength-selective filter by a tapered fiber, which is followed by an isolator and a polarization controller. Light from the output of the filter is collected by another tapered fiber and coupled back to the input of the EDF. The tap port of a $1 \times 2$ coupler with a nominal splitting ratio of 1:99 as the laser output was used.

The overall fiber-chip-fiber insertion loss was measured to be $\sim 15 \mathrm{~dB}$, higher than previously reported $10.4 \mathrm{~dB}$ of a very similar structure in ref. [42], mainly due to observed imperfections of the fabricated waveguides and nanotapers. Round-trip losses include additional contributions from the polarization controller and the $1 \times 2$ coupler which are measured to be $0.6 \mathrm{~dB}$ and $1.0 \mathrm{~dB}$, respectively. The gain obtained from EDF at $\lambda=1.55 \mu \mathrm{m}$, which exceeds $20 \mathrm{~dB}$ as shown in Figure 6.9 (inset) overcomes the losses stated above; therefore lasing was expected in this band with the proposed silicon microring filter.

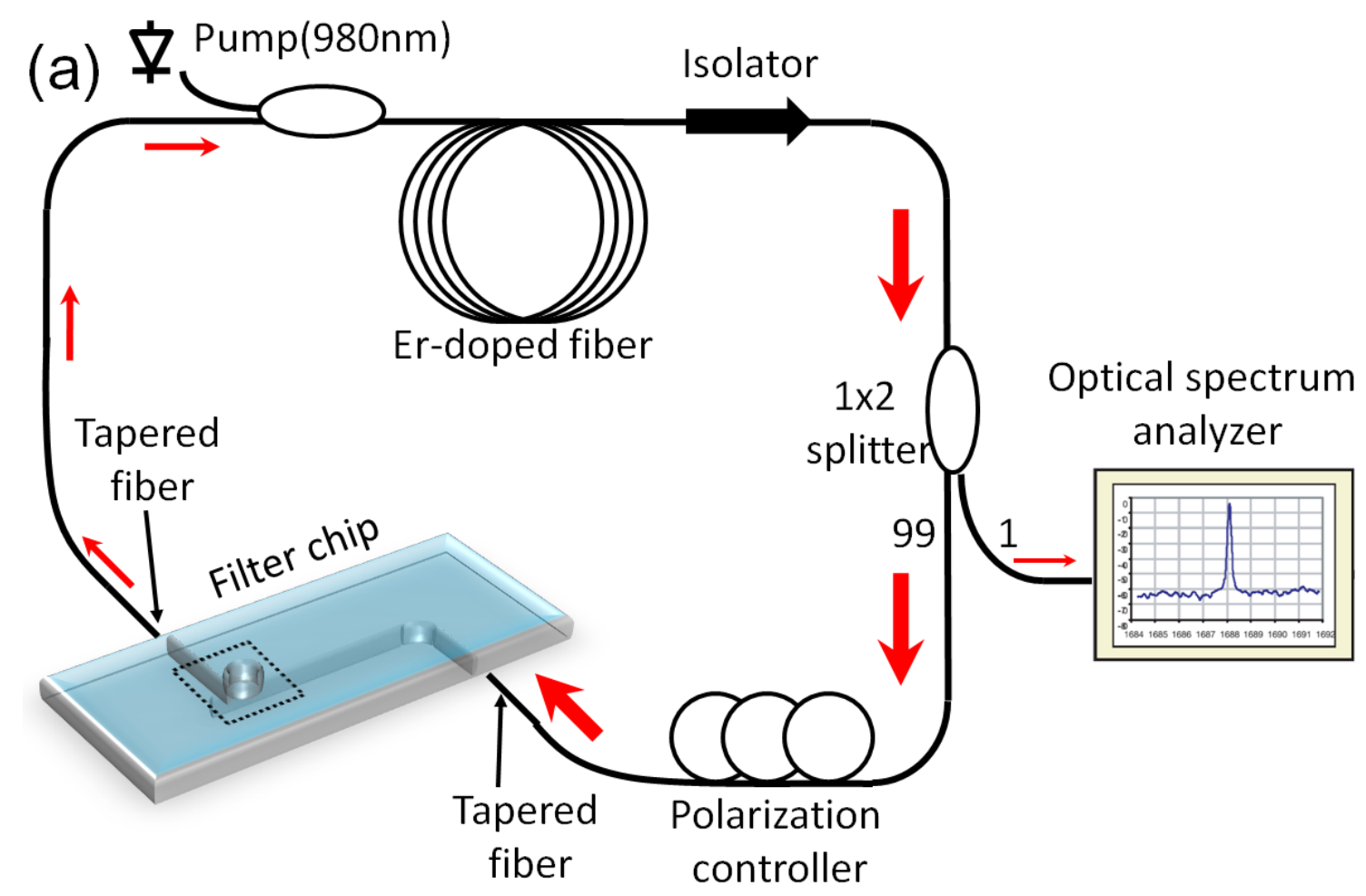




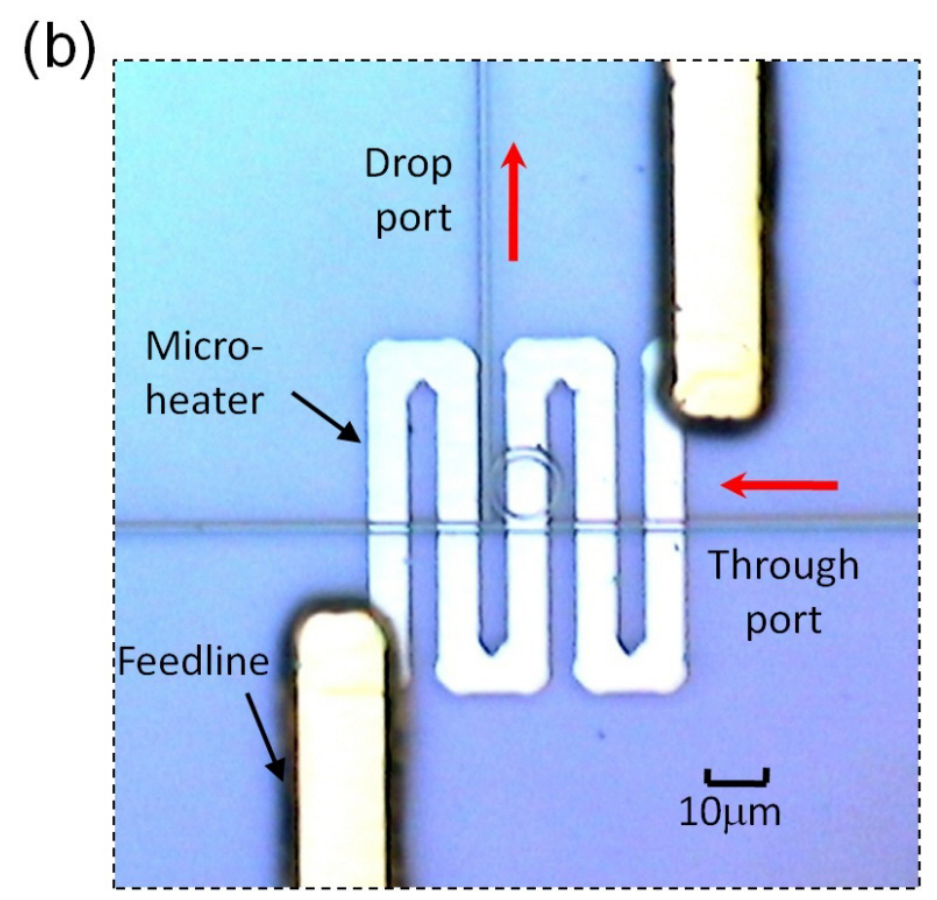

Figure 6.6 (a) Experimental setup of the tunable laser (b) top view microscopic picture of fabricated switch element

The birefringent nature of the microring resonator leads to separated quasi-TE resonances and quasi-TM resonances observed within a FSR of the filter [51]. Thus, the filter transmission spectrum is sensitive to input polarization. Our device is optimized at quasi-TE resonances with a much narrower linewidth $(\mathrm{FWHM}=0.1-0.2 \mathrm{~nm})$ compared to quasi- $\mathrm{TM}$ ones $(\mathrm{FWHM}=1-2 \mathrm{~nm})$. Figure 6.7(a) shows the transmission spectrum around $1.55 \mu \mathrm{m}$ of the filter that was taken using the broadband unpolarized ASE source when the chip was at room temperature $\left(24^{\circ} \mathrm{C}\right)$. The quasi-TE and quasi-TM resonance wavelengths were measured to be $1555.56 \mathrm{~nm}$ and $1550.93 \mathrm{~nm}$ respectively. Similar peak transmissions and extinction ratios were achieved for both quasi-TE and quasi-TM resonances. The extinction ratios were measured to be over $10 \mathrm{~dB}$. The double resonance characteristic of the TE peak has been attributed to sidewall back scattering on the microring [46]. By tuning the input polarization state using the polarization controller, the 
polarization-dependent loss can be controlled to switch on/off the TE or TM lasing modes. Figure $6.7(\mathrm{~b}, \mathrm{c})$ shows lasing spectra at $1555.57 \pm 0.01 \mathrm{~nm}$ or $1550.90 \pm 0.1 \mathrm{~nm}$ corresponding to the quasi-TE or quasi-TM filter resonances, respectively. Simultaneous operation at two-wavelengths is demonstrated by adjusting the input polarization at $45^{\circ}$ to the chip normal with lasing observed at both TE and TM wavelengths (Figure 6.7(d)). Significantly narrower FWHMs of $0.02 \mathrm{~nm}$ were observed in both lasing modes, approaching the resolution limit of the optical spectrum analyzer $(0.01 \mathrm{~nm})$. The lasing wavelength of the TM mode was observed to shift $\pm 0.1 \mathrm{~nm}$ around $1550.90 \mathrm{~nm}$ due to the broad peak linewidth of the quasi-TM resonance of the filter. However, for the TE mode, the lasing wavelength shift was $\pm 0.01 \mathrm{~nm}$, which is significantly reduced, compared to TM lasing mode and approaches the wavelength resolution of the OSA. An interesting phenomenon was observed when comparing the top-view infrared images captured of the microring filter at corresponding lasing modes as shown in the insets of Figure 6.7(b-d). An infrared camera was used to monitor and capture the top view image with exactly the same camera settings. From the images, greater scattering around the ring area was observed when lasing at the TE mode compared to the TM mode although slightly higher lasing output was measured at the TM mode. This is due to the increased off-plane scattering of TE mode with electric field parallel to the surface of the sample. The speckle pattern observed around the filter area is due to reflections from the unpolished bottom surface of the chip. The TM mode preferentially scatters along the plane of the sample, hence the reduced intensity collected by the top viewing camera. Other filter resonances within EDF gain bandwidth are found at $1537.45 \mathrm{~nm}$ (quasi-TE) and $1533.81 \mathrm{~nm}$ (quasi-TM), however, lasing was never observed on those wavelengths 
even with highest EDF pumping current provided. This is because the corresponding gain at those wavelengths are at least $6 \mathrm{~dB}$ lower than at $1550 \mathrm{~nm}$ band for our EDF and thus below the lasing threshold.

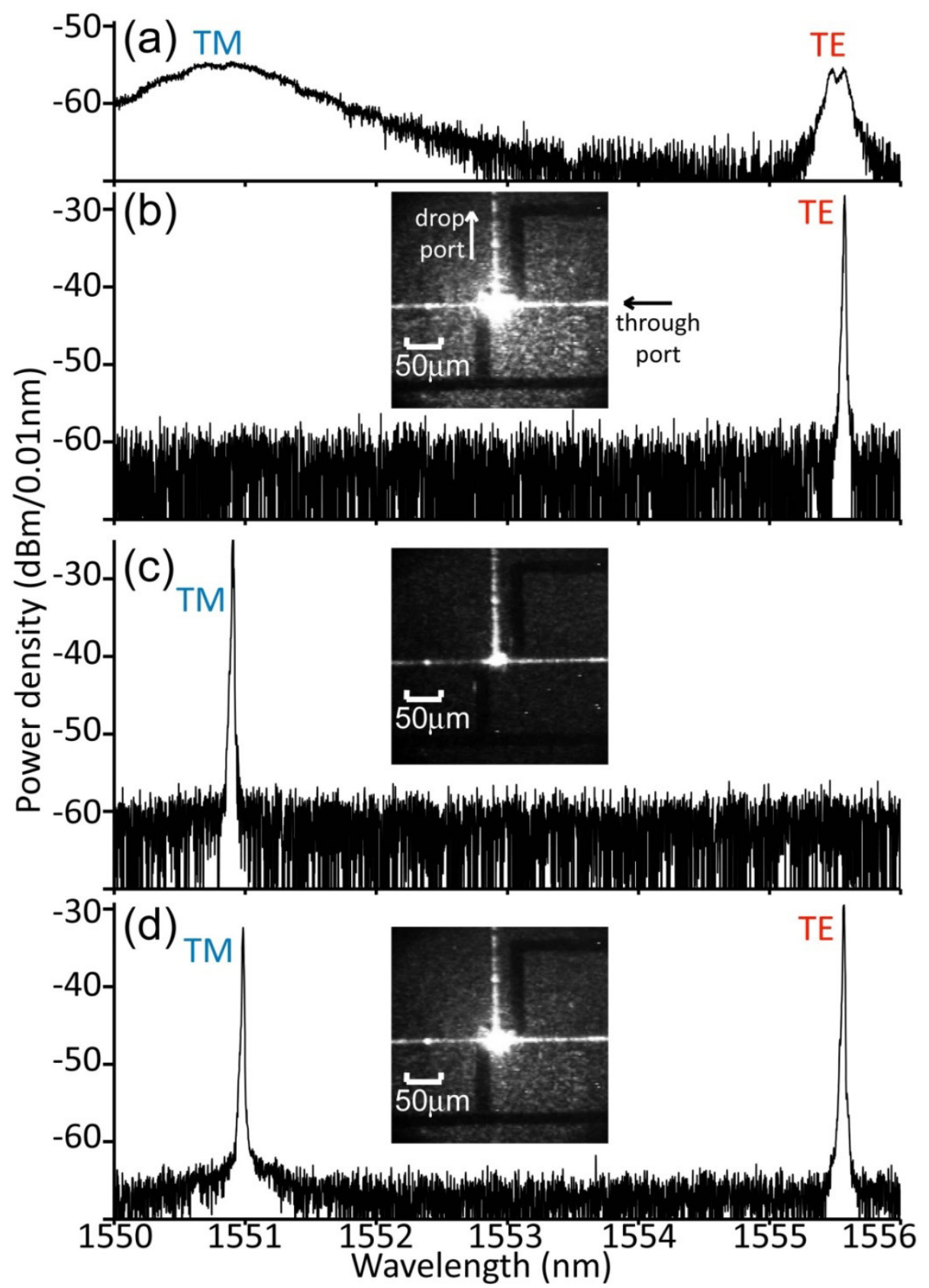

Figure 6.7 (a) Transmission spectrum of the filter using unpolarized ASE source. Lasing spectra of: (b) TE mode; (c) TM mode; and (d) simultaneous lasing of both TE and TM modes. Insets show corresponding top view infrared images of the ring area during lasing. 


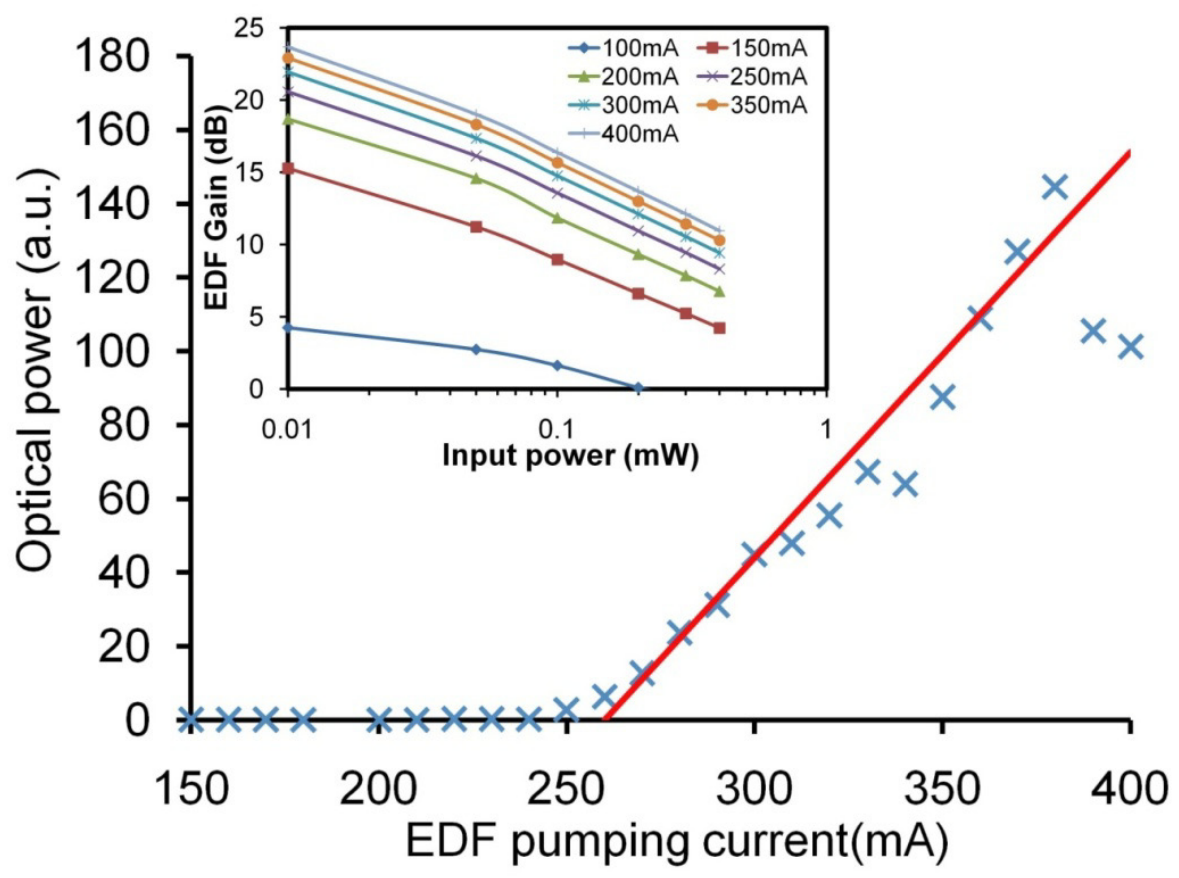

Figure 6.8 Laser output power versus the EDF pumping bias current. Inset shows the EDF gain at $1550 \mathrm{~nm}$ for different input power at pumping current from $100 \mathrm{~mA}$ to $400 \mathrm{~mA}$

Figure 6.8 plots the laser output power versus the EDF pump bias current with the tuning filter chip maintained at room temperature. In this experiment, the input polarization state was adjusted by a manual polarization controller that was set to $\mathrm{TE}$ mode at the highest pumping current. The pump current was decreased to below threshold and an ANDO AQ6317B optical spectrum analyzer (OSA) was used to record the emission spectra. The peak position and power were noted at each pump current. From the plot we can determine a lasing threshold of $\sim 260 \mathrm{~mA}$. We estimate the power in the loop after the EDF to be $\sim 0.3 \mathrm{~mW}$ with pumping current of $400 \mathrm{~mA}$. The small value is due to the high losses in our current silicon tunable filter chip. It is important to note that losses in silicon photonics have been shown to be as low as $3.6 \pm 0.1 \mathrm{~dB} / \mathrm{cm}[52]$. The 
output power was observed to fluctuate significantly for pumping currents above 300 mA, however, lasers built with same EDF and FBG grating filters showed a stable output. This effect is attributed to fluctuations losses in the fiber-to-chip coupling.

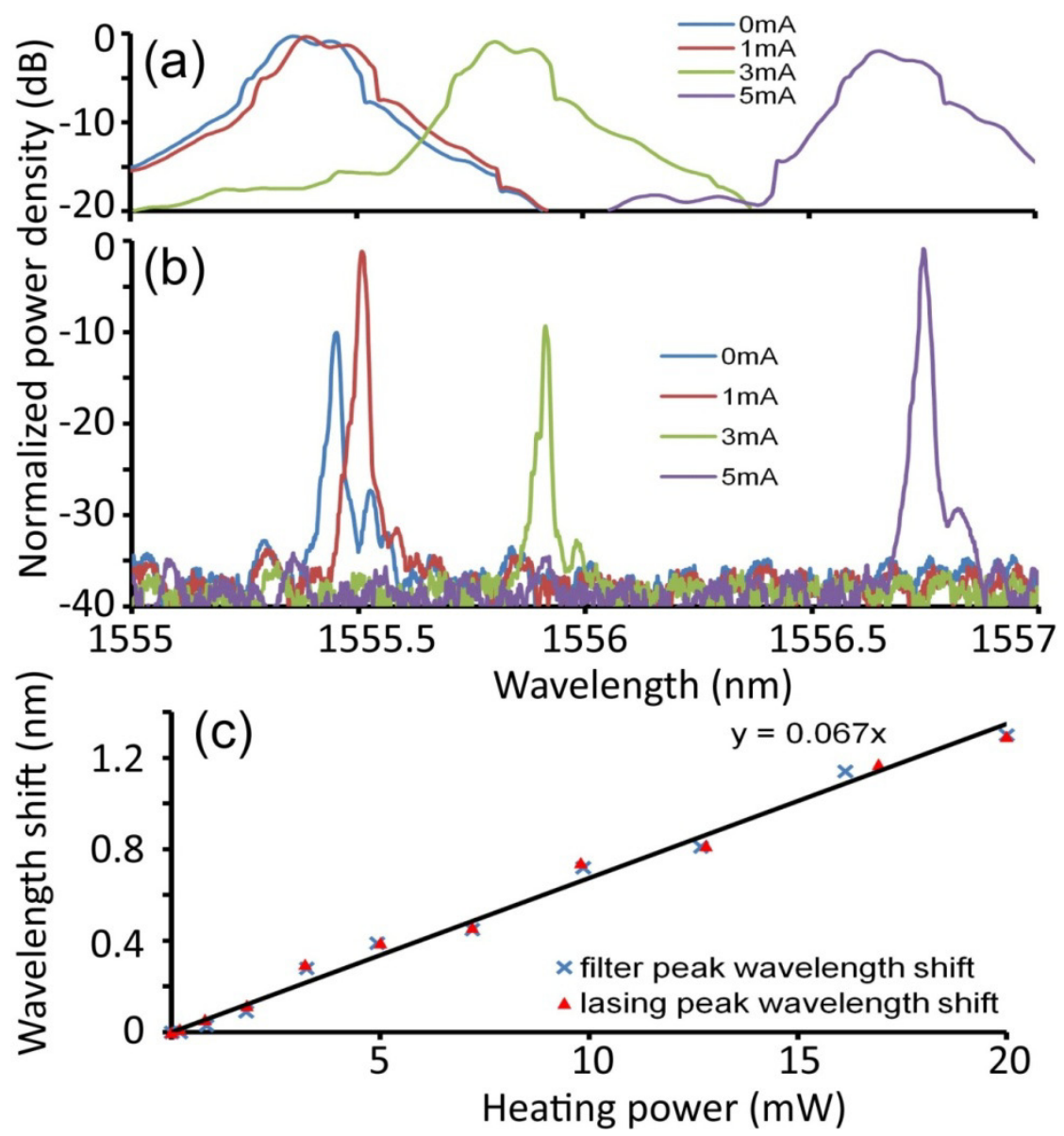

Figure 6.9 (a) Filter spectra and (b) lasing spectra at applied heating current of $0,1,3$ and $5 \mathrm{~mA}$ (c) filter and lasing peak wavelength shift versus applied heating power.

Tuning of the lasing wavelength was achieved by adjusting the current into the micro-heater to tune the silicon microring resonance. Previously we demonstrated pulsed operation of the silicon microring tunable filter up to $6.4 \mathrm{~nm}$ [37]. However, continuous operation is restricted to $5 \mathrm{~mA}$ at which point the probe contacts are not reliable. Figure 6.9(a,b) show the filter transmission spectra and the laser emission spectra taken at the 
same microheater currents of $0,1,3$ and $5 \mathrm{~mA}$ after thermal equilibrium had been reached. Both spectra were found to red-shift with constant current heating as previously demonstrated [43]. The peak shift was found to be proportional to the electrical heating power as seen in Figure 6.10(c) which shows wavelength shifts versus applied heating power with a measured slope of $\sim 0.067 \mathrm{~nm} / \mathrm{mW}$. These results agree with the thermooptic effect as demonstrated in [37]. Lasing wavelengths were found to closely match the peak transmission wavelengths of the filter and following the shift. A tunable range of over $1.3 \mathrm{~nm}$ has been demonstrated with low heating power of $20 \mathrm{~mW}$ although much wider tunable range is achievable.

In summary, we have demonstrated a new application of silicon photonics for the control of on-chip wavelengths through the use of an external gain source. Tuning of 1.3 $\mathrm{nm}$ was demonstrated with $20 \mathrm{~mW}$ electrical power, with wider tuning ranges of at least up to $6.4 \mathrm{~nm}$ possible with larger currents. Simultaneous lasing at two different wavelengths was achieved using the natural birefringence of the devices. The use of parallel ring configurations will enable simultaneous lasing wavelengths to be selected and tuned across the whole Er+ range. This integration of the wavelength control of optical sources into silicon photonics is an important step towards DWDM applications. 


\section{CONCLUSION AND FUTURE WORK}

A thermally wavelength-tunable photonic filter/switch was designed based on silicon microring resonator. The large FSR $(\sim 18 \mathrm{~nm})$, sharp filter passband $(0.1-0.2 \mathrm{~nm})$ and wide tunable range $(>6.4 \mathrm{~nm})$, enables DWDM applications with the device. The tunable range was highest on a single silicon microring to the best of our knowledge until its publishing date. The device provides a scalable approach for high port count optical routing with a profitable architecture. With compact footprint $(50 \mu \mathrm{m} \times 50 \mu \mathrm{m}, 10 \mu \mathrm{m}$ $\times 10 \mu \mathrm{m}$ for optimized heater design), low power consumption (on the order of $\sim 10 \mathrm{~mW}$ with S-shape heater and $\sim \mathrm{mW}$ expected with optimized heater design) and full compatibility with SOI technology, a large number of these devices can be integrated on a single silicon chip which is promising for low cost production. The time response of the switch is on the order of $\sim 10 \mu \mathrm{s}$.

Two important and novel applications were demonstrated in this work, the onchip $4 \times 4$ reconfigurable routing, and the silicon on-chip laser wavelength tuning. The former makes possible high port count DWDM application up to at least 32 input and output ports. The latter work demonstrated on-chip wavelength control in a silicon photonics platform for the first time, and is a very promising approach to realize independent controlled multi-wavelength lasing on chip using a common gain media. The demonstrated applications are significant as they are fundamental in establishing a silicon-based platform for silicon photonics towards DWDM applications that will apply to all types of optical communication, including on-chip interconnects. 
Future work of this project includes improvement of device performance, implementation of new functions, and demonstration of other applications.

According to this work and other studies, there is improvement space of the device performance including: 1) larger FSR and smaller footprint with reduced ring diameter [4]; 2) wider tunable range [38]; 3) lower power consumption and faster speed. The microring resonator platform also offers design flexibility for implementing functions desirable for optical communication applications such as high speed modulation [3], tunable bandwidth ROADM [40], hitless operation [66, 62], and flat-top and fast roll-off filter for telecomm applications[67,41].

With the wavelength-tunable filter, we will work on multi-wavelength laser wavelength tuning and tracking of external laser wavelength. 


\section{LIST OF REFERENCES}

[1] L. C. Kimerling, MIT Communications Technology Roadmap, Cambridge, MA (2005).

[2] L. Chen, P. Dong, and M. Lipson, "High performance germanium photodetectors integrated on submicron silicon waveguides by low temperature wafer bonding," Opt. Express 16, 11513-11518 (2008)

[3] Q. Xu, B. Schmidt, S. Pradhan, and M. Lipson, "Micrometre-scale silicon electrooptic modulator," Nature 435, 325-327 (2005).

[4] Q. Xu, D. Fattal, and R. G. Beausoleil, "Silicon microring resonators with 1.5- $\mu \mathrm{m}$ radius," Opt. Express 16, 4309-4315 (2008)

[5] B. E. Little, S. T. Chu, H. A. Haus, J. Foresi, and J.-P. Laine, "Microring resonator channel dropping filters," J. Lightw. Technol., vol. 15, no. 6, pp. 998-1005, Jun. 1997.

[6] B. E. Little, S. T. Chu, P. P. Absil, J. V. Hryniewicz, F. G. Johnson, F. Seifert,D. Gill, V. Van, O. King, and M. Trakalo, "Very high-order microring resonator filters for WDM applications," IEEE Photon. Technol. Lett., vol. 16, no. 10, pp. 2263-2265, Oct. 2004.

[7] F. Vollmer, S. Arnold, D. Braun, I. Teraoka, and A. Libchaber, "Multiplexed DNA quantification by spectroscopic shift of two microsphere cavities," Biophysical Journal, vol. 85, pp. 1974-9, 2003.

[8] A. M. Armani and K. J. Vahala, "Heavy water detection using ultra-high-Q microcavities," Optics Letters, vol. 31, pp. 1896-8, 2006.

[9] G. Lenz, B. J. Eggleton, C. K. Madsen, C. R. Giles, and G. Nykolak, "Optimal dispersion of optical filters for WDM systems," IEEE Photonics Technology Letters, vol. 10, pp. 567-9, 1998.

[10] C. K. Madsen, J. A. Walker, J. E. Ford, K. W. Goossen, T. N. Nielsen, and G. Lenz, "A tunable dispersion compensating MEMS all-pass filter," IEEE Photonics Technology Letters, vol. 12, pp. 651-3, 2000.

[11] X. Liu, W. Fang, Y. Huang, X. H. Wu, S. T. Ho, H. Cao, and R. P. H. Chang, "Optically pumped ultraviolet microdisk laser on a silicon substrate," Applied Physics Letters, vol. 84, pp. 2488-90, 2004. 
[12] K. Djordjev, S.-J. Choi, S.-J. Choi, and P. D. Dapkus, "Vertically coupled InP microdisk switching devices with electroabsorptive active regions," IEEE Photonics Technology Letters, vol. 14, pp. 1115-17, 2002.

[13] K. Djordjev, S.-J. Choi, S.-J. Choi, and P. D. Dapkus, "Gain trimming of the resonant characteristics in vertically coupled InP microdisk switches," Applied Physics Letters, vol. 80, pp. 3467-9, 2002.

[14] G. N. Nielson, D. Seneviratne, F. Lopez-Royo, P. T. Rakich, F. Giacometti, H. L. Tuller, and G. Barbastathis, "MEMS based wavelength selective optical switching for integrated photonic circuits," presented at Conference on Lasers and Electro- Optics (CLEO). IEEE. Part vol.1, 2004, pp. 2. Piscataway, NJ, USA.

[15] P. Rabiei, W. H. Steier, Z. Cheng, C.-g. Wang, and H. J. Lee, "Polymer microring modulator with $1 \mathrm{THz}$ FSR," presented at Postdeadline Papers. Summaries of papers presented at the Conference on Lasers and Electro-Optics. Conference Edition (IEEE Cat. No.02CH37337). Opt. Soc. America. Part vol.2, 2002, pp. CPDB8-1-CPDC2-3. Washington, DC, USA.

[16] S. T. Chu, B. E. Little, V. Van, J. V. Hryniewicz, P. P. Absil, F. G. Johnson, D. Gill, O. King, F. Seiferth, M. Trakalo, and J. Shanton, "Compact full C-band tunable filters for $50 \mathrm{GHz}$ channel spacing based on high order micro-ring resonators," presented at Optical Fiber Communication Conference (OFC) (IEEE Cat. No.04CH37532). Opt. Soc. America. Part vol.2, 2004, pp. 3. Washington, DC, USA.

[17] H. C. Tapalian, J. P. Laine, and P. A. Lane, "Thermooptical switches using coated microsphere resonators," IEEE Photonics Technology Letters, vol. 14, pp. 1118- 20, 2002.

[18] T. Sadagopan, S. June Choi, K. Djordjev, and P. D. Dapkus, "Carrierinduced refractive index changes in InP-based circular microresonators for low-voltage highspeed modulation," IEEE Photon. Technol. Lett., vol. 17, no. 2, pp. 414-416, Feb. 2005.

[19] K. Djordjev, S.-J. Choi, S.-J. Choi, and R. D. Dapkus, "Microdisk tunable resonant filters and switches," IEEE Photonics Technology Letters, vol. 14, pp. 828-30, 2002.

[20] V. R. Almeida, C. A. Barrios, R. R. Panepucci, and M. Lipson, "All-optical control of light on a silicon chip," Nature 431, 1081-1084 (2004).

[21] W. von Klitzing, R. Long, V. S. Illcenko, J. Hare, and V. Lefevre-Seguin, "Frequency tuning of the whispering-gallery modes of silica microspheres for cavity quantum electrodynamics and spectroscopy," Optics Letters, vol. 26, pp. 166-8, 2001.

[22] A. L. Huston and J. D. Eversole, "Strain-sensitive elastic scattering from cylinders," Optics Letters, vol. 18, pp. 1104-6, 1993. 
[23] V. S. Ilchenko, P. S. Volikov, V. L. Velichansky, F. Treussart, V. Lefevre-Seguin, J. M. Raimond, and S. Haroche, "Strain-tunable high-Q optical microsphere resonator," Optics Communications, vol. 145, pp. 86-90, 1998.

[24] E. Pawlowski, K. Takiguchi, M. Okuno, K. Sasayama, A. Himeno, K. Okamoto, and Y. Ohmori, "Variable bandwidth and tunable centre frequency filter using transversalform programmable optical filter," Electron. Lett. 32, 113-114 (1996).

[25] W. M. J. Green, R. K. Lee, G. A. DeRose, A. Scherer, and A. Yariv, "Hybrid InGaAsP-InP Mach-Zehnder racetrack resonator for thermooptic switching and coupling control," Optics Express, vol.13, no.5, 7 March 2005.

[26] J. Yao, D. Leuenberger, M. M. Lee, M. C. Wu, "Silicon Microtoroidal Resonators with MEMS Tunable Coupler", IEEE Journal of Selected Topics in Quantum Electronics, Vol. 13, No. 2, pp. 202-208, Mar./Apr. 2007

[27] M. R. Watts, "Wavelength switching and routing through evanescently induced absorption," Master's thesis, Massachusetts Institute of Technology, Cambridge, MA, 2001.

[28] E. A. J. Marcatili, “Optical frequency filters using disk cavity,”26 Jan. 1971, US Patent 3,558,213.

[29] P. T. Rakich, M. A. Popoviф, M. R. Watts, T. Barwicz, H. I. Smith, and E. P. Ippen, "Ultrawide tuning of photonic microcavities via evanescent field perturbation," Optics Letters, vol. 31, no. 9, pp. 1241-1243, May 2006.

[30] U. Levy, K. Campbell, A. Groisman, S. Mookherjea, and Y. Fainman, "On-chip microfluidic tuning of an optical microring resonator," Applied Physics Letters, vol. 88, pp. 111107-1-3, 2006.

[31] J. C. Palais, Fiber optic communications (fifth edition), Pearson Prentice Hall, 2005.

[32] AllWave optical fiber, OFS Fitel, Norcross, Georgia

[33] "Excerpts from A Conversation with Gordon Moore: Moore's Law", Intel Corporation. 2005. pp. 1

[34] J. Wagener and T. Strasser, "Characterization of the economic impact of stranded bandwidth in fixed OADM relative to ROADM networks," presented at the Optical Fibre Communication Conf./National Fiber Optic Engineers Conf. (OFC/NFOEC), Anaheim, CA, Mar. 5-10, 2006, Paper OThM6. 
[35] R. L. Espinola, M. C. Tsai, J. T. Yardley, and R. M. Osgood Jr., "Fast and lowpower thermooptic switch on thin silicon-on-insulator," IEEE Photon. Technol. Lett., vol. 15, no. 10, pp. 1366-1368, Oct. 2003.

[36] J.Li, A.Q.Liu, Q.X.Zhang, N.Balasubramanian, and C.Lu,"A Hybrid reconfigurable OADM using MEMS switch and FBG", Proc. SPIE, 2002, 4907, pp. 9-15

[37] X. Wang, J. A. Martinez, M.S. Nawrocka and R.R. Panepucci, "Compact thermally tunable silicon wavelength switch: modeling and characterization," IEEE Photon. Technol. Lett. 20, 936-938 (2008).

[38] F. Gan, T. Barwicz, M. A. Popovic, M. S. Dahlem, C. W. Holzwarth, P. T. Rakich, H. I. Smith, E. P. Ippen, and F. X. Kärtner, "Maximizing the thermo-optic tuning range of silicon photonic structures", in Proceeding of IEEE Conference on Photonics in Switching (San Francisco, CA, 2007), pp. 67-68.

[39] M.-Y. Park, J.-H. Kim, H.-K. Lee, S.-M. Cho, S.-K. Hong, Y.-G. Lee, Y.-S. Oh, and J.-B. Hong, "Development of small port-count ROADM using MEMS type 2 x 2 OSWs and VOAs," in Proc. Optical MEMS and Their Applications Conf., Aug. 1-4, 2005, pp. $153-154$.

[40] L.Chen, N. Sherwood-Droz, and M. Lipson, "Compact bandwidth-tunable microring resonators," Opt. Lett. 32, 3361-3363 (2007)

[41] M. Popovic, 'Theory and design of high-index-contrast microphotonic resonators and circuits,' Ph.D. dissertation (MIT, 2008).

[42] Q. Xu, V. R. Almeida, and M. Lipson, "Micrometer-scale all-optical wavelength converter on silicon," Opt. Lett. 30, 2733-2735 (2005).

[43] M. S. Nawrocka, T. Liu, X. Wang, and R. R. Panepucci, "Tunable silicon microring resonator with wide free spectral range," Appl. Phys. Lett. 89, 071110-071113, (2006).

[44] V. R. Almeida, R. R. Panepucci, and M. Lipson, "Nanotaper for compact mode conversion," Opt. Lett. 28, 1302-1304 (2003).

[45] "Heat Transfer Module-User’s Guide”, Comsol Multiphysics, version 3.3 (2006)

[46] B. E. Little, J. -P. Laine, and S. T. Chu, "Surface-roughness-induced contradirectional coupling in ring and disk resonators," Opt. Lett. 22, 4-6 (1997)

[47] H. Ng, M. R. Wang, D. Li, X. Wang, J. Martinez, R. R. Panepucci, and K. Pathak, "1x4 wavelength reconfigurable photonic switch using thermally tuned microring resonators fabricated on silicon substrate," IEEE Photon. Technol. Lett. 19, 704 -706 (2007). 
[48] H. Ng, M. R. Wang, D. Li, X. Wang, J. A. Martinez, R. R. Panepucci, and K. M. Pathak, "4 x 4 wavelength-reconfigurable photonic switch based on thermally tuned silicon microring resonators," Optical Engineering 47, 044601 (2008).

[49] O. Boyraz and B. Jalali, "Demonstration of a silicon Raman laser," Opt. Express 12, 5269-5273 (2004).

[50] H. Rong, A. Liu, R. Jones, O. Cohen, D. Hak, R. Nicolaescu, A. Fang, and M. Paniccia, "An all-silicon Raman laser," Nature 433, 292-294 (2005)

[51] J. T. A. Carriere, J. A. Frantz, B. R. Youmans, S. Honkanen, and R. K. Kostuk, "Measurement of waveguide birefringence using a ring resonator," IEEE Photon. Technol. Lett. 16, 1134-1136 (2004)

[52] Y. Vlasov and S. McNab, "Losses in single-mode silicon-on-insulator strip waveguides and bends," Opt. Express 12, 1622-1631 (2004)

[53] L. Eldada, J. Fujita, A. Radojevic, T. Izuhara, R. Gerhardt, J. Shi, D. Pant, F. Wang, and A. Malek, " 40-Channel Ultra-Low-Power Compact PLC-Based ROADM Subsystem," in Optical Fiber Communication Conference and Exposition and The National Fiber Optic Engineers Conference, Technical Digest (CD) (Optical Society of America, 2006), paper NThC4.

[54] JDS Uniphase Corporation,"40 channel integrated PLC reconfigurable optical add/drop multiplexer",

http://www.jdsu.com/product-literature/40plcroadm_ds_cms_ae_020806.pdf

[55] J.Ertel, R.Helbing, C.Hoke, O.Landolt, K.Nishimura, et al.,"Design and performance of a reconfigurable liquid-crystal-based optical add/drop multiplexer", IEEE/OSA J. Light. Tech., 2006, 24, (4), pp. 1674-1680

[56] B. E. Little, S. T. Chu, H. A. Haus, J. Foresi, and J.-P. Laine, "Microring Resonator Channel Dropping Filters," IEEE J. Lightwave. Technology 15, 998-1005 (1997).

[57] A. Yariv, "Universal relations for coupling of optical power between microresonators and dielectric waveguides," Electron. Lett., vol. 36, pp. 321, Feb.,2000

[58] D. G. Rabus, Integrated Ring Resonators: The Compendium (Springer, Berlin, 2007).

[59] Quality factor, wikipedia, http://en.wikipedia.org/wiki/Quality_factor 
[60] Q. Xu, V. R. Almeida, R. R. Panepucci, and M. Lipson, "Experimental demonstration of guiding and confining light in nanometer-size low-refractive-index material," Opt. Lett. 29, 1626-1628 (2004)

[61] L. Zhou, K. Kashiwagi, K.Okamoto, R. P. Scott, N. K. Fontaine, D. Ding, V. Akella, S. J. B. Yoo, "Towards athermal optically-interconnected computing system using slotted silicon microring resonators and RF-photonic comb generation", Applied Physics A: Materials Science \& Processing, Vol. 95, No. 4, pp. 1101-1109, June, 2009.

[62] N. Sherwood-Droz, H. Wang, L. Chen, B. G. Lee, A. Biberman, K. Bergman, and M. Lipson, "Optical 4x4 hitless slicon router for optical networks-on-chip (NoC)," Opt. Express 16, 15915-15922 (2008)

[63] LPCVD Stoichiometric Nitride Characterization, Cornell Nanofabrication Facility (CNF), Ithaca, NY http://www.cnf.cornell.edu/doc/LPCVDStoichioNiOct2004.pdf

[64] C. Pollock and M. Lipson, Integrated Photonics, Kluwer Academic, Dordrecht, 2003.

[65] X. Wang, T. Liu, V. R. de Almeida, and R. R. Panepucci, “On-chip silicon photonic wavelength control of optical fiber lasers," Optics Express 16, 15671-15676 (2008).

[66] M.A. Popovic, T. Barwicz, F. Gan, M.S. Dahlem, C.W. Holzwarth, P.T. Rakich, H.I. Smith, E.P. Ippen and F.X. Kartner, "Transparent wavelength switching of resonant filters," presented at Conference on Lasers and Electro-Optics (CLEO), Baltimore, MD, May 10, 2007, postdeadline paper CPDA2.

[67] S. Xiao, M. H. Khan, H. Shen, and M. Qi, "A highly compact third-order silicon microring add-drop filter with a very large free spectral range, a flat passband and a low delay dispersion," Opt. Express 15, 14765-14771 (2007)

[68] E. J. Klein, D. H. Geuzebroek, H. Kelderman, G. Sengo, N. Baker, and A. Driessen, "Reconfigurable Optical Add-Drop Multiplexer Using Microring resonator, IEEE Photonics Technology Letters, vol. 17, issue 11, pp. 2358-2360 2005

[69] I. Christiaens, D. Van Thourhout, and R. Baets, "Low-power thermo-optic tuning of vertically coupled microring resonators," Electronics Letters, vol. 40, no. 9, pp. 560-561, Apr. 2004.

[70] P. Rabiei, W. H. Steier, C. Zhang, and L. R. Dalton, "Polymer micro-ring filters and modulators," J. Lightwave Technol., vol. 20, no. 11, pp. 1968-1975, Nov. 2002.

[71] Y. Yanagase, S. Yamagata, and Y. Kokubun, "Wavelength tunable polymer microringresonator filter with 9.4nm tuning range," Electron. Lett. 39922-924 (2003) 
[72] D.H.Geuzebroek, E.J.Klein, H.Kelderman and A.Driessen, "Wavelength tuning and switching of a thermo-optic microring resonator", proc. ECIO 2003, pp. 395-398, 2003.

[73] T. Wang, C. Chu, and C. Lin, "Electro-optically tunable microring resonators on lithium niobate," Opt. Lett. 32, 2777-2779 (2007)

[74] R. Grover, T. A. Ibrahim, S. Kanakaraju, L. Lucas, L. C. Calhoun, and P.-T. Ho, "A tunable GaInAsP-InP optical microring notch filter," IEEE Photon. Technol. Lett.,vol. 16, no. 2, pp. 467-469, Feb. 2004.

[75] T. A. Ibrahim, W. Cao, Y. Kim, J. Li, J. Goldhar, P.-T. Ho, C. H. Lee, "All-optical switching in a laterally coupled microring resonator by carrier injection," IEEE Photonics Technology Letters, vol. 15, pp. 36-38, 2003

[76] L. Eldada and L. W. Shacklette, "Advances in polymer integrated optics," IEEE J.Select. Topics Quantum Electron., vol. 6, no. 1, pp. 54-68, Jan. 2000.

[77] T. Miya, "Silica-based planar lightwave circuits: passive and thermally active devices," IEEE J. Select. Topics Quantum Electron., vol. 6, no. 1, pp. 38-45, Jan. 2000.

[78] V. Almeida and M. Lipson, "Optical bistability on a silicon chip," Opt. Lett. 29, 2387-2389 (2004)

[79] T. Tsuchizawa, K. Yamada, H. Fukuda, T. Watanabe, J. Takahashi, M. Takahashi, T. Shoji, E. Tamechika, S. Itabashi, and H. Morita, "Microphotonics devices based on silicon micro-fabrication technology," IEEE J. Sel. Top. Quantum Electron. 11, 232-240 (2005).

[80] S. Xiao, M. H. Khan, H. Shen, and M. Qi, "Compact silicon microring resonators with ultra-low propagation loss in the C band," Opt. Express 15, 14467 (2007)

[81] V. Padgaonkar, The 2004 NNIN REU Research Accomplishments, 2004, pp. 98-99, http://www.nnin.org/doc/2004NNINreuRA.pdf

[82] M.W. Geis, S.J. Spector, R.C. Williamson and T.M. Lyszczarz, "Submicrosecond submilliwatt silicon-oninsulator thermooptic switch, " IEEE Photon. Technol. Lett. 16, 2514-2516 (2004).

[83] M. W. Pruessner, T. H. Stievater, M. S. Ferraro, and W. S. Rabinovich, "Thermooptic tuning and switching in SOI waveguide Fabry-Perot microcavities," Opt. Express $15,7557-7563$ (2007).

[84] M. Harjanne, M. Kapulainen, T. Aalto, and P. Heimala, "Sub- $\mu$ S Switching Time in Silicon-on-Insulator. Mach-Zehnder Thermooptic Switch," IEEE Photon. Technol. Lett., VOL. 16, NO. 9, 2039, Sept. 2004.. 
[85] I. Kiyat, A. Aydinli, and N. Dagli, "Low-Power Thermooptical Tuning of SOI Resonator Switch,” IEEE. Photon. Technol. Lett. 18, 364-366 (2006)

[86] M. El-Sayed and J. Jaffe, "A view of telecommunications network evolution," IEEE Commun. Mag., vol. 40, no. 12, pp. 74-81, Dec. 2002.

[87] K. Kudo, T. Morimoto, K. Yashiki, T. Sasaki, Y. Yokoyama, K.Hamamoto, and M. Yamaguchi, "Wavelength-selectable microarray light sources of multiple ranges simultaneously fabricated on single wafer," Electron. Lett., vol. 36, no. 8, pp. 745-747, 2000.

[88] S. -L. Lee, I. -F. Jang, C. -Y.Wang, C. -T. Pien, and T. -T. Shih, "Monolithically integrated multiwavelength sampled grating lasers for dense WDM applications," IEEE J. Select. Topics Quantum Electron., vol. 6, no. 1, pp. 197-206, 2000.

[89] M. Aijada, K. Alameh, and Y. T. Lee," A Tunable Multiwavelength Laser Employing a Semiconductor Optical Amplifier and an Opto-VLSI Processor", IEEE Photon. Technol. Lett., 20(10), pp.815-817 (2008)

[90] M. S. Wu, E. C. Vail, G. S. Li, W. Yuen, and C. J. Chang-Hasnain,” Widely and continuously tunable micromachined resonant cavity detector with wavelength tracking", IEEE Photon. Tech. Lett., vol. 8, pp. 98-100, Jan. 1996

[91] D.R.Cassidy, G.H. Cross, "Picometer Resolution Wavelength Tracking in the C Band Using an InP-InGaAsP Dual-Slab Interferometer", IEEE Photon. Tech. Lett., vol. 19, pp. 1075-1077, Jul 2007 


\section{XUAN WANG}

March 26, 1980

2002

2002-2003

2003-2004

2004-2005

2005 to present
Born, Wuhan, China

B.S., Optoelectronics Engineering

Huazhong University of Science and Technology

Wuhan, China

R\&D Engineer

Comcore Technology, Inc.

Shanghai, China

Teaching Assistant

Department of Physics

University of Miami

Miami, Florida

Teaching Assistant

Department of Electrical and Computer Engineering Florida International University

Miami, Florida

Research Assistant

Nanophotonics Research Group

Florida International University

Miami, Florida

Dissertation Year Fellowship Award

\section{PUBLICATIONS AND PRESENTATIONS}

\section{Journal Publications}

X. Wang, T. Liu, V. R. de Almeida, and R. R. Panepucci, "On-chip silicon photonic wavelength control of optical fiber lasers," Optics Express 16, 15671-15676 (2008).

X. Wang, J. A. Martinez, M.S. Nawrocka and R.R. Panepucci, "Compact thermally tunable silicon wavelength switch: modeling and characterization," IEEE Photon. Technol. Lett. 20, 936-938 (2008).

H. Ng, M. R. Wang, D. Li, X. Wang, J. A. Martinez, R. R. Panepucci, and K. M. Pathak, " 4 x 4 wavelength-reconfigurable photonic switch based on thermally tuned silicon microring resonators," Optical Engineering 47, 044601 (2008). 
H. Ng, M. R. Wang, D. Li, X. Wang, J. Martinez, R. R. Panepucci, and K. Pathak, "1x4 wavelength reconfigurable photonic switch using thermally tuned microring resonators fabricated on silicon substrate," IEEE Photon. Technol. Lett. 19, 704 -706 (2007).

M. S. Nawrocka, T. Liu, X. Wang, and R. R. Panepucci, "Tunable silicon microring resonator with wide free spectral range," Appl. Phys. Lett. 89, 071110-071113, (2006).

\section{Selected Conference Presentations}

X. Wang, T. Liu, and R. R. Panepucci, On-chip silicon photonic wavelength control of laser source, Photonics West 2009, San Jose, CA, Jan 2009.

X. Wang, T. Liu, V. R. Almeida, and R. R. Panepucci, Fiber Laser Wavelength Tuning on a Silicon Chip, Optical Fiber Communication Conference, OSA Technical Digest (CD) (Optical Society of America, 2009), paper JThA11.

M. R. Wang, H. Ng, D. Li, X. Wang, J. Martinez, R. R. Panepucci, and K. Pathak, Wavelength reconfigurable photonic switching using thermally tuned micro-ring resonators fabricated on silicon substrate, Proc. SPIE 6645, 66450I (2007).

M. S. Nawrocka, X. Wang, T. Liu, J. Martinez, R. R. Panepucci.,"Nanophotonics for Communications and Biosensing", Poster presented on the Optical Waveguide Sensing \& Imaging in Medicine, Environment, Security and Defence Workshop, October $12-21$, 2006, Gatineau, Québec, Canada, organized by NATO-Advanced Study Institute

H. Ng, M. R. Wang, X. Wang, J. Martinez, R. R. Panepucci, D.Li, 8x8 Wavelength Reconfigurable Photonic Switch Using Thermally Tuned Micro-Ring Resonators Fabricated on Silicon Substrate, Frontiers in Optics 2006, Rochester, NY., October 8-12, 2006

R. R. Panepucci, V. R. Almeida, X. Wang, A. Lavrenov, A. M. P. Fievre, Sensing using nano-opto-electromechanical systems (NOEMS), International Microelectronics and Packaging Society (IMAPS) Emerging Technology Workshop on Nanotechnologies for Microelectronics, Las Vegas, NV, December 4-7, 2006.

X. Wang, M.S. Nawrocka, I. Kartadjodjaja, V. Almeida, M. Lipson, B. Ilic, R. Panepucci, Silicon cantilever-wavelength displacement sensors, The 49th Interational Conference on Electron, Ion and Photon Beam Technology \& Nanofabrication, Grande Lakes, Orlando, Florida, May 31 - June 3, 2005 